Florida International University FIU Digital Commons

FIU Electronic Theses and Dissertations

University Graduate School

$11-20-2003$

\title{
Exclusive electrodisintegration of three-nucleon systems
}

Tigran Abrahamyan

Florida International University

DOI: $10.25148 /$ etd.FI13101511

Follow this and additional works at: https://digitalcommons.fiu.edu/etd

Part of the Physics Commons

\section{Recommended Citation}

Abrahamyan, Tigran, "Exclusive electrodisintegration of three-nucleon systems" (2003). FIU Electronic Theses and Dissertations. 1081. https://digitalcommons.fiu.edu/etd/1081

This work is brought to you for free and open access by the University Graduate School at FIU Digital Commons. It has been accepted for inclusion in FIU Electronic Theses and Dissertations by an authorized administrator of FIU Digital Commons. For more information, please contact dcc@fiu.edu. 
FLORIDA INTERNATIONAL UNIVERSITY

Miami, Florida

EXCLUSIVE ELECTRODISINTEGRATION OF THREE-NUCLEON SYSTEMS

A thesis submitted in partial fulfillment of the requirements for the degree of MASTER OF SCIENCE in

\section{PHYSICS}

by

Tigran Abrahamyan 
To: Dean R. Bruce Dunlap

College of Arts and Sciences

This thesis, written by Tigran Abrahamyan, and entitled Exclusive Electrodisintegration of Three-Nucleon Systems, having been approved in respect to style and intellectual content, is referred to you for judgment.

We have read this thesis and recommend that it be approved.

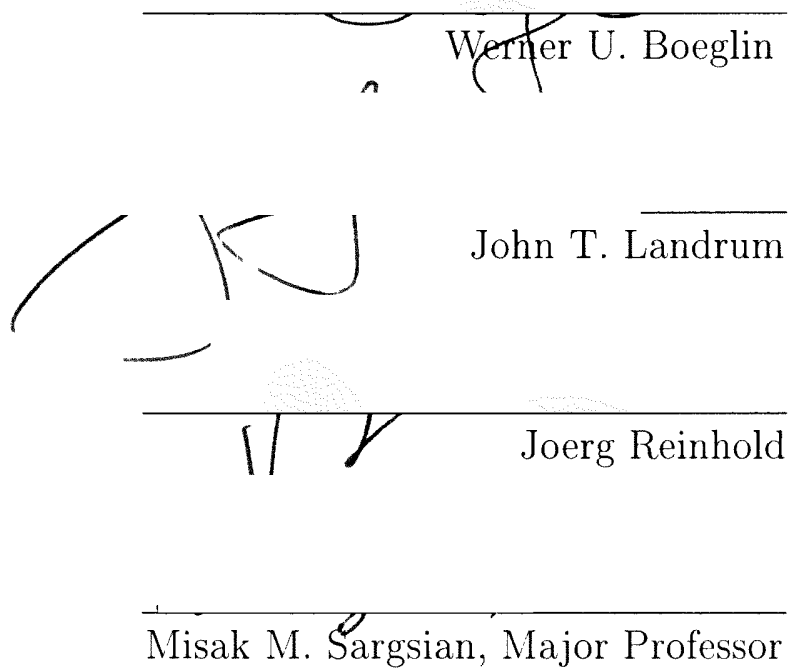

Date of Defense: November 20, 2003

The thesis of Tigran Abrahamyan is approved.
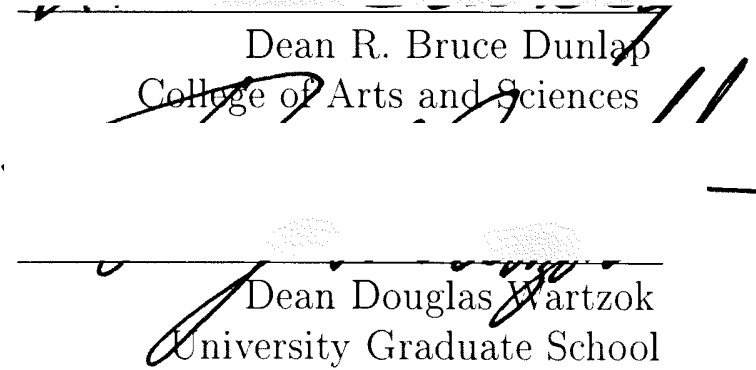

Florida International University, 2003 


\section{ACKNOWLEDGMENTS}

I would like to express my deepest gratitude to my advisor Dr. Misak M. Sargsian for his guidance, support and encouragement throughout my graduate education. His continuous efforts to broaden my knowledge in theoretical physics are sincerely appreciated by me.

I am very grateful to my committee members Dr. Werner U. Boeglin, Dr. John T. Landrum and Dr. Joerg Reinhold for reading the manuscript and their comments.

I also wish to thank Dr. Richard A. Arndt from the George Washington University for the help with nucleon-nucleon partial-wave analysis numerical package. I am thankful to Dr. Rustam Niyazov from the Old Dominion University for provided experimental data.

Finally, many thanks to all the faculty and staff of the Florida International University Physics Department for their constant support during the course of my graduate studies. 


\section{ABSTRACT OF THE THESIS}

\section{EXCLUSIVE ELECTRODISINTEGRATION OF THREE-NUCLEON SYSTEMS by}

Tigran Abrahamyan

Florida International University, 2003

Miami, Florida

Professor Misak M. Sargsian, Major Professor

The purpose of this research was to develop a theory of high-energy exclusive electrodisintegration of three-nucleon systems on the example of ${ }^{3} \mathrm{He}\left(e, e^{\prime} N N\right) N$ reaction with knocked-out nucleon in the final state.

The scattering amplitudes and differential cross section of the reaction were calculated in details within the Generalized Eikonal Approximation(GEA). The manifestly covariant nature of Feynman diagrams derived in GEA allowed us to preserve both the relativistic dynamics and kinematics of the scattering while identifying the low momentum nuclear part of the amplitude with a nonrelativistic nuclear wave function. Numerical calculations of the residual system's total and relative momentum distribution were performed which show reasonable agreement with available experimental data.

The theoretical framework of GEA, which was applied previously only for the case of two-body (deuteron) high energy break up reactions, has been practically implemented and shown to provide a valid description for more complex $A=3$ systems. 


\section{TABLE OF CONTENTS}

CHAPTER

PAGE

1 Introduction 1

2 General Kinematic Requirements, Emergence of Small Parameters $\quad 7$

3 Some Basic Features of Exclusive Electro-Nuclear Reactions 10

4 Final State Interactions 15

5 Reduction Theorem 17

6 Feynman Diagram Rules for the Scattering Amplitude in GEA 23

7 Scattering Amplitudes for ${ }^{3} \mathrm{He}\left(e, e^{\prime} N N\right) N$ Reaction 27

7.1 Plane Wave Impulse Approximation and Pair Distortion Amplitudes . 27

7.2 Single Rescattering Amplitudes . . . . . . . . . . . . . . . . 36

7.3 Double Rescattering Amplitudes . . . . . . . . . . . . . . . 46

8 Differential Cross Section 53

8.1 General Formula . . . . . . . . . . . . . . . . . . . 53

8.2 Nuclear Decay Function . . . . . . . . . . . . . . . . 57

9 Numerical Estimates $\quad 59$

9.1 Subroutine eheppn . . . . . . . . . . . . . . . . . . . 59

9.2 Comparison with Experiment . . . . . . . . . . . . . 60

$\begin{array}{ll}10 \text { Conclusions } & 67\end{array}$

$\begin{array}{lc}\text { LIST OF REFERENCES } & 69\end{array}$

$\begin{array}{ll}\text { APPENDICES } & 70\end{array}$ 
1 High energy electrodisintegration of the three-nucleon system. . . . 7

$2 \quad$ Four main contributing processes. . . . . . . . . . . . . 10

3 The $\left|p_{m z}\right|$ as a function of $\mathrm{x} \ldots \ldots \ldots \ldots \ldots \ldots \ldots$

4 The $p_{m}$ dependence of the differential cross sections of $d\left(e, e^{\prime} p\right) n \ldots \ldots 13$

5 The $Q^{2}$ dependence of $W_{1} / W_{2}$ from inclusive $d\left(e, e^{\prime}\right) X$ reaction. . . . 14

6 Proton-proton and proton-neutron total cross sections. . . . . . . . 15

$7 \quad$ Diagram for hadron-deuteron scattering . . . . . . . . . . . 17

8 Reduction theorem for hadron-deuteron scattering. . . . . . . . . . 22

9 Diagram for $n$-fold rescattering. . . . . . . . . . . . . . . . 24

10 Impulse Approximation Diagram. . . . . . . . . . . . . . . . . . 27

11 Single Rescattering Diagrams. . . . . . . . . . . . . . . . 36

12 Double Rescattering Diagrams. . . . . . . . . . . . . . 46

13 Total cross section for proton-neutron charge-exchange scattering. . . 54

14 Relative and total momentum distribution of NN pairs at $2.2 \mathrm{GeV}$. $\quad 64$

$152.2 \mathrm{GeV}$ data and J.-M. Laget's calculations. . . . . . . . . . . . . 65

16 Relative and total momentum distribution of NN pairs at $4.4 \mathrm{GeV}$. $\quad 66$

17 Poles on the complex plain. . . . . . . . . . . . . . . 79

18 Integral over $d \mu=d(\cos \theta) \ldots \ldots \ldots \ldots$. . . . . . . . . 80 


\section{Introduction}

In the 70's the theory of Quantum Chromodynamics emerged as a consistent field theory of strong interaction. The quarks were shown to be constituent blocks of strongly interacting particles - hadrons, while carriers of strong interactions are known to be the gluons. Quantum Chromodynamics introduces a new phenomenon - confinement, which is most probably due to self interaction of the gluons. Because of the confinement quarks and gluons are not detected in free isolated states. Very little is known about how quarks and gluons generate the nuclear force which attracts protons and nucleons in atomic nuclei.

Strongly interacting few-body systems are considered the best micro-laboratory where the mechanism of the generation of strong forces through quarks and gluons can be investigated. Presently, our understanding of the subnuclear structure is limited to excitation energies much smaller than the nucleon mass and to the dynamics of single-nucleon states characterized by the momenta $\leq 200-250 \mathrm{MeV} / \mathrm{c}$. Nuclear dynamics at very short distances, when nucleons are strongly overlapping each other, remain almost unexplored $[1,2]$. One knows from experimental studies of nuclear disintegrations that it is possible to detect nucleons carrying momenta as high as $750 \mathrm{MeV} / \mathrm{c}$. Naive application of Heisenberg's uncertainty principle suggests that at these values of momenta instantaneous hadronic densities can be up to eight times larger than in regular nuclear configurations. These are densities characteristic for neutron stars (for comparison the density of such stars is known to 
be $\left.9.1 \times 10^{16} \mathrm{~kg} / \mathrm{m}^{3}\right)$. In order to probe such small space-time distances in nuclear structure one has to provide high energy and momentum transfer to the nucleus.

The electro-nuclear reactions are best suited for high momentum and energy transfer processes since the electron coupling to the virtual photon can be precisely calculated in QED. In addition, the well known fact from particle physics that with increasing energies theoretical descriptions of photon-hadron interactions become simpler and more reliable (see e.g. Ref.[4]) would be relevant also for electro-nuclear reactions.

Few-body systems, such as ${ }^{3} \mathrm{He}$ (consisting of two protons and one neutron), are advantageous for theorists in the sense that there exist well established quantummechanical methods of solving the three body problem for structureless particles interacting within a given local potential [3]. Furthermore, consideration of the quark-gluon structure of few nucleons is significantly simplier than for heavier nuclei. This is also true for the problems related to the relativistic generalization of the system.

In addition to the increase of the transferred momenta and energies, an increase of the degrees of exclusiveness of electro-nuclear reactions, when more products are registered in the final state of the reaction, allows us to attain deeper understanding of the dynamics of the reaction as well as to gain more information about the microscopic structure of short-range nucleon correlations [1].

The combination of these two factors: high energy and momentum transfer and exclusiveness of the reaction makes electro-nuclear reactions a unique laboratory for 
studying nuclear quantum chromodynamics.

During the last decade there was a tremendous growth in the number of proposed and performed experiments dedicated to exclusive nuclear reactions with large values of momentum transfer $(\geq 1 \mathrm{GeV} / \mathrm{c}$ ) (see e.g. [5]). The experimental studies of high momentum transfer exclusive reactions are an important part of the scientific programs of the high energy, high intensity electron facilities at Jefferson Lab, USA and HERMES, DESY, Germany. Therefore the development of an adequate theoretical framework to describe these reactions is becoming a pressing problem.

The major issue facing the theoretical description of exclusive reactions is that when the final state of the reaction, in addition to the scattered electron, consists of at least one hadron the strong reinteraction of the produced hadrons in the nuclear environment becomes the dominant feature of these reactions.

One of the issues in describing these reinteractions is that the theoretical methods which were successful in medium-energy nuclear physics should be upgraded in order to describe hadronic re-interactions in the processes in which energies transferred to a nuclear target are large $(\sim$ few $\mathrm{GeV})[6]$.

At energies of the produced hadronic system $E_{N} \leq 1 \mathrm{GeV}$ the final state interactions(FSI) in exclusive nuclear reactions are usually evaluated in terms of an effective potential for the interaction in the residual system - the optical model approximation. Two important features of high-energy FSI make the extension of the potential formalism to high energies problematic. Firstly, the number of essential partial waves increases rapidly with the energy of the $N(A-1)$ system. Secondly, the $N N$ interac- 
tion which is practically elastic for $E_{N} \leq 500 \mathrm{MeV}$ becomes predominantly inelastic for $E_{N}>1 G e V$. Hence the problem of scattering cannot be treated as a many body quantum mechanical problem. In this situation introducing a predominantly imaginary potential to account for hadron production is not a well defined mathematical concept. So, theoretical methods successful below $1 \mathrm{GeV}$ become ineffective at the transferred momenta which can be reached at Jefferson Lab and HERMES.

Final state reinteraction in hadron induced nuclear reactions at higher energies $\left(E_{N}>1 \mathrm{GeV}\right)$ are often described within the approximation of the additivity of phases, acquired in the sequential rescatterings of high-energy projectiles off the target nucleons (Glauber model [7]). This approximation made it possible to describe the data on elastic $h A$ scattering at hadron energies $1 \mathrm{GeV}<E_{h}<10-15 \mathrm{GeV}$.

There are many theoretical works that have appeared in the last several years where the same principle of Glauber rescattering has been applied to the description of the cross sections of $A\left(e, e^{\prime} N\right)(A-1)$ reactions. Many of these works discussed the reactions in which the cross sections were integrated over the excitation energies of the residual nuclear system and the kinematics were restricted to small momenta of the residual system, $\vec{p}_{A-1} \leq p_{F}$, where $p_{F} \approx 250 \mathrm{MeV} / \mathrm{c}$ is the characteristic Fermi momentum of the nuclear system. In all these cases the Glauber approximation, which considers the nucleons in the nuclei as stationary scatterers, was a good approximation.

However the Glauber approximation cannot be applied to the class of $e A$ reactions in which the main emphasis is given to the high momentum of the bound 
nucleon and high excitation energies of the residual nuclei. This situation is especially important in studies of short-range nuclear properties in which large values of missing momentum and excitation energy are involved. It is also important in studies of the transparency of the nuclear medium for high momentum transfer electro-production reactions.

In this thesis we present the Final State Interactions (FSI) calculations for exclusive ${ }^{3} \mathrm{He}\left(e, e^{\prime} N N\right) N$ reactions within the Generalized Eikonal Approximation(GEA) 6 , 2]. Unlike other high-energy approximation models (e. g. Glauber model, [7]) GEA takes into account the preexisting momenta of nucleons in nuclei. GEA is a relativistic approximation which allows us to rewrite a potentially infinite number of final state hadronic reinteractions into a finite set of covariant Feynman diagrams. One can actually calculate and extract all the FSI contributions, reconstructing momentum distributions initially present in the ${ }^{3} \mathrm{He}$ nucleus which is the starting point of any short range correlation study.

We will concentrate on the kinematical region where the bound nucleon momenta and excitation energies in the nuclear system are large enough that shortrange multinucleon correlations are expected to be dominant in the nuclear wave function. We are particularly interested in the region of the transferred four momenta $1 \leq Q^{2} \leq 6 \mathrm{GeV}^{2}$. Here the lower limit is defined by the condition that the knocked-out hadronic system is energetic enough such that high energy computational approaches become applicable. The upper limit is defined from the conditions that color coherence effects are small and the produced hadronic state represents a 
single state (e.g. nucleon) but not the superposition of different hadronic states (wave packet). Thus we have a unique kinematic window where the theoretical methods of high energy scattering may have a simpler realization due to the fact that mainly diagonal (elastic) terms in $N N$ rescattering will dominate. 


\section{General Kinematic Requirements, Emergence}

\section{of Small Parameters}

In this thesis we are considering exclusive electrodisintegration of three-body systems on the example of the ${ }^{3} \mathrm{He}$ nucleus. The corresponding reaction is given by:

$$
e+{ }^{3} \mathrm{He} \rightarrow e^{\prime}+p+p+n
$$

and shown schematicly in Figure 1. The discussion throughout assumes that high momentum $q \equiv k_{e}-k_{e^{\prime}}=\left(q_{0}, \mathbf{q}\right)$ is transferred to the ${ }^{3}$ He nucleus. The main feature of the final state in these reactions is that it contains a fast nucleon that carries almost the entire momentum of the virtual photon $\mathbf{p}_{\mathrm{f}} \approx \mathbf{q}$, with $|\mathbf{q}| \sim$ several $G e V / c$. The two other hadrons $\left(p_{r}\right.$ and $\left.p_{s}\right)$ in the final state have relatively low energy $\left(p_{r, s} \sim\right.$ hundreds $\left.M e V / c\right)$.

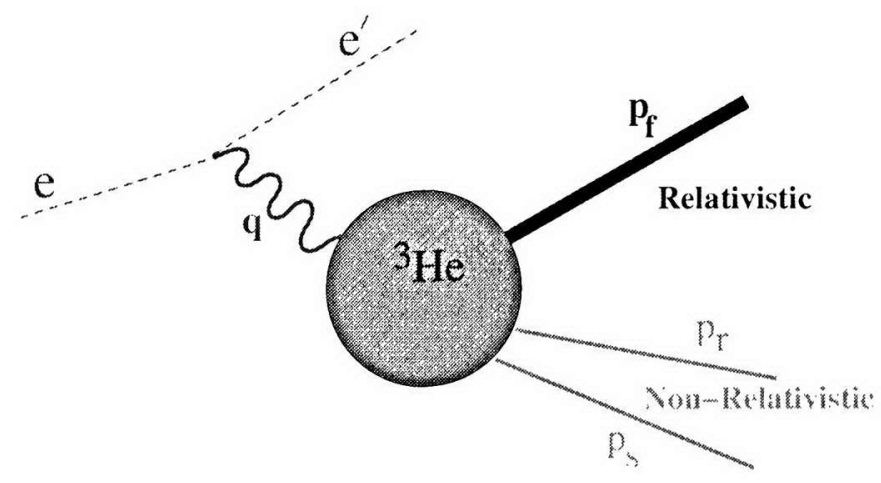

Figure 1: High energy electrodisintegration of the three-nucleon system.

Hereafter the $z$ direction is defined by $\mathbf{q}$ and the scattering plane is the plane of $\mathbf{q}$ and $\mathbf{k}_{e^{\prime}}$ vectors. 
Thus the major kinematic requirements are the following:

$$
\begin{aligned}
\text { (a) } 6 \mathrm{GeV}^{2} \geq & Q^{2} \geq 1 \mathrm{GeV}^{2}, \\
\left(\text { b) } \mathbf{p}_{\mathbf{f}}\right. & \approx \mathbf{q}, \\
\text { (c) } p_{f} \gg p_{m}, p_{r}, p_{s} & \sim \text { few hundreds } \mathrm{MeV} / \mathrm{c} .
\end{aligned}
$$

where $\mathbf{p}_{\mathbf{m}}=\mathbf{p}_{\mathbf{f}}-\mathbf{q}$ is the missing momentum of the reaction. We define the missing energy as $E_{m}=m+E_{A-1}-M_{A}$, which characterizes the excitation of the residual nuclear system ( $m$ is the mass of the nucleon). Note that our definition of $E_{m}$ differs from the commonly used definition of missing energy for which the kinetic energy of the center of mass of the $A-1$ system is subtracted. However for our discussions this difference is not important.

The lower limit in eq.(2(a)) is the main requirement that provides a high-momentum transfer in the electrodisintegration. This condition together with eqs.(2(b)) and (c) allows us to identify the $p_{f}$ as a knocked-out nucleon, while $p_{r}$ and $p_{s}$ can be considered as recoil nucleons which do not interact directly with the virtual photon. The upper limit in eq.(2(a)) is defined from the condition that the color coherence effects are small and the produced hadronic state represents a single state (i. e. nucleon) but not a superposition of different hadronic states in the form of a wave packet. Also, due to the conditions established by eq. $(2(c))$ the nucleons are the basic degrees of freedom describing the interacting nuclear system. From the technical point of view this means that for the set of noncovariant diagrams, comprising the covariant scattering amplitude, one can neglect the noncovariant diagrams containing 
non-nucleonic degrees of freedom (e.g. negative energy projections of bound nucleon spinors or vacuum fluctuations). In this case one can reduce the nuclear vertices to the nonrelativistic nuclear wave functions of nuclei.

Another consequence of our kinematics is the availability of certain small parameters. If one constructs the \pm components of four-momenta (which correspond to the energy and longitudinal momentum of the particle in the light-cone reference frame) $p_{ \pm}=p_{0} \pm p_{z}$, where $z$ direction is defined by the direction of virtual photon momentum q, then one observes that the condition of Eq.(2) corresponds to the smallness of the following combinations:

$$
\frac{q_{-}}{q_{+}} \approx \frac{m x}{2 q} \ll 1 \text { and } \frac{p_{f-}}{p_{f+}} \approx \frac{m^{2}}{4 p_{f}^{2}} \ll 1
$$

were $x=Q^{2} / 2 m q_{0}$. The availability of these small parameters is one of the important features of high energy scattering as compared to the low-intermediate energy reactions. We will see below how these conditions will simplify the theoretical treatment of semi-exclusive three-nucleon electrodisintegration reactions. 


\section{Some Basic Features of Exclusive}

\section{Electro-Nuclear Reactions}

The first exclusive electro-nuclear reactions which have been studied at intermediate energies confirmed the expectations that the complexity of the hadronic system significantly restricts the unambiguous treatment of the underlying dynamics of the reaction. In general, four main processes are contributing to the exclusive electronuclear reactions (Figure 2), in which at least one energetic nucleon is registered in the final state:

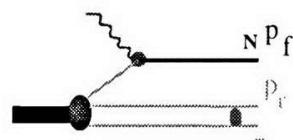

(a) IA

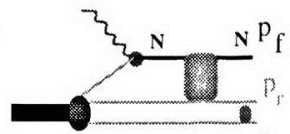

(b) FSI

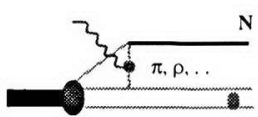

(c) MEC

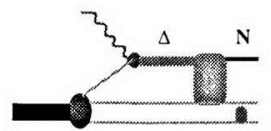

(d) IC

Figure 2: Impulse Approximation (a), final state interaction (b), meson exchange (c), and $\Delta$-isobar contribution (d) diagrams.

- Impulse approximation (IA) amplitude (Figure 2a), in which the virtual photon knocks-out the bound nucleon which propagates to the final state without further interactions,

- Final state interaction (FSI) amplitude (Figure 2b), in which the knocked-out nucleon reinteracts with the residual hadronic system,

- Amplitude with meson exchange currents (MEC) (Figure 2c), in which the 
virtual photon interacts with the exchanged (between two-nucleon system) mesons,

- Isobar current contribution amplitude (IC) (Figure 2d), in which the virtual photon produces a $\Delta$-isobar which reinteracts with the residual nuclear system producing the final hadronic state.

The study of the small distance properties of nuclei in these reactions is related to the exploration of the IA diagram at high values of missing momenta and energy. However for such kinematics at small $Q^{2}\left(\ll 1 \mathrm{GeV}^{2}\right)$, practically in all cases the FSI, MEC and IC give dominant contributions[8].

There are several reasons for the dominance of FSI, MEC and IC diagrams in the kinematics of large missing momentum $p_{m}$, missing energy $E_{m}$ and low $Q^{2}$. While at large $p_{m}$ and $E_{m}$ the IA amplitude is defined by the nuclear wave function at short inter-nucleon distances, the FSI, MEC and IA amplitudes are defined by the nuclear wave function of the average configurations.

The dominance of MEC and IC amplitudes follows also from the kinematical considerations that at small $Q^{2}$ high missing momenta $p_{m}$ in exclusive $A\left(e, e^{\prime} N\right) X$ reactions can be observed only at $x<1$, i.e. in the kinematic region close to the pion threshold. It can be seen from Figure 3, where we calculated the $\left|p_{m z}\right|$ dependence on $x$ for quasi-elastic $e+d \rightarrow e^{\prime}+p+n$ reaction, that at $Q^{2}<1 G e V^{2}$ only $x<1$ is appropriate for detection of large $\left|p_{m z}\right| \geq 300 \mathrm{MeV} / \mathrm{c}$.

What concerns the final state interactions at small $Q^{2}$, they are dominated by 
$S$ wave scattering and have broad angular distributions. Thus there exist no clear criteria how to isolate or suppress FSI with respect to IA.

In general terms the dominance of FSI, MEC and IA amplitudes means the impossibility to probe small space-time intervals in the nucleus using probes of larger size $(1 / q \geq 1 \mathrm{fm})$.

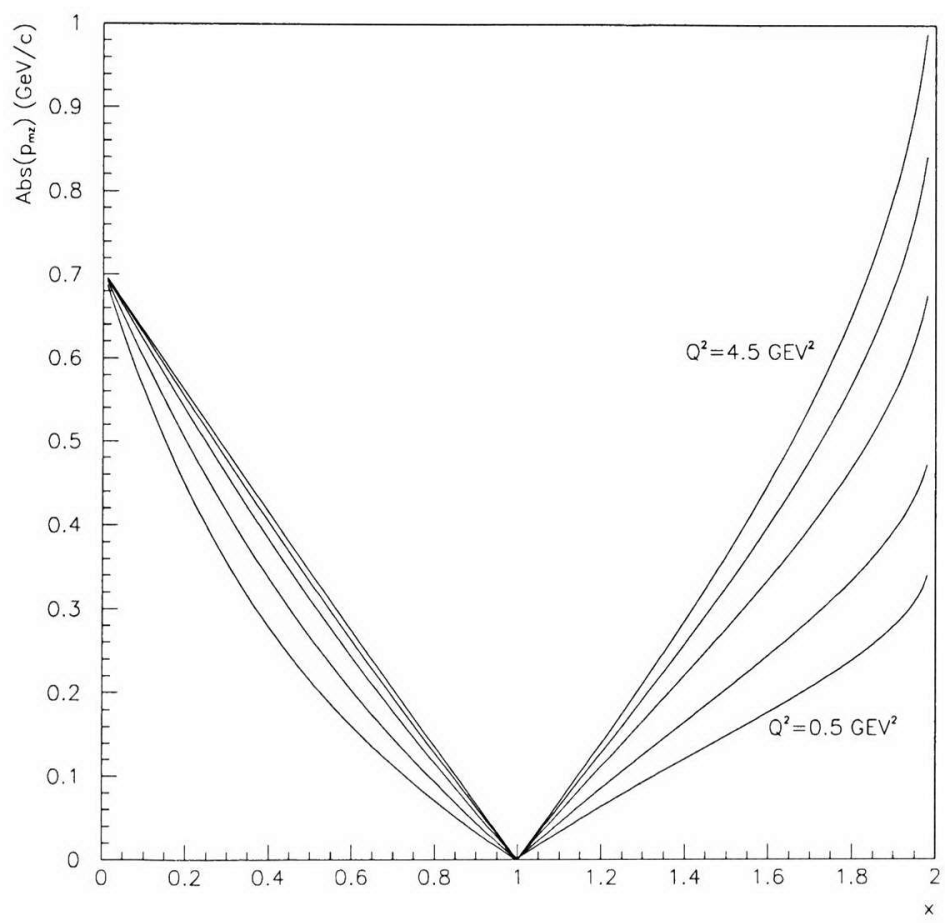

Figure 3: The $\left|p_{m z}\right|$ as a function of $\mathrm{x}$, for different values of $Q^{2}$, for quasielastic $d\left(e, e^{\prime} N\right) N$ reaction. The lines between $Q^{2}=0.5 G e V^{2}$ and $Q^{2}=4.5 G e V^{2}$ curves correspond to the values of $Q^{2}=1.5,2.5,3.5 \mathrm{GeV}^{2}$.

In Figure 4 we present the typical intermediate energy measurement, which demonstrates how large MEC and IC contributions are in the cross section at large 
missing momenta and low $Q^{2}$. The calculations in the kinematic region of these experiments show that at large $p_{m}$ MEC and IC significantly dominate the PWIA contribution.

With the increase of energy the situation changes qualitatively. It may sound paradoxical but at high energy transfer, when the wavelength of the probe becomes much smaller than the sizes of interacting particles the situation becomes simpler.

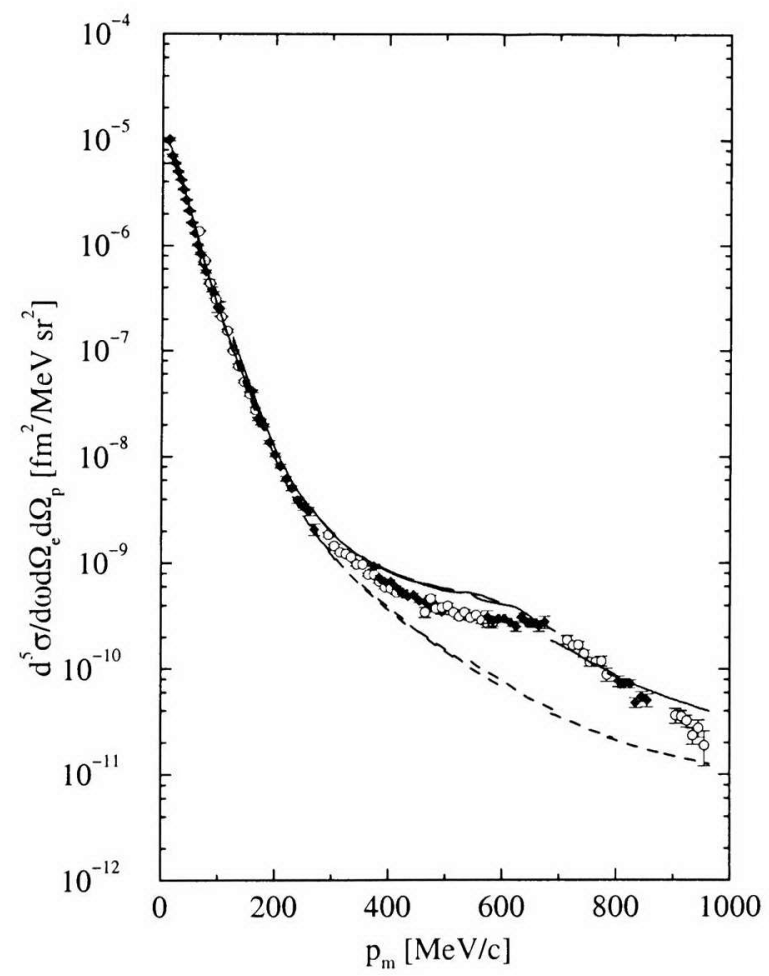

Figure 4: The $p_{m}$ dependence of the differential cross sections of $d\left(e, e^{\prime} p\right) n$ reactions at $Q^{2}=0.13-0.33 \mathrm{GeV}^{2}$. The data are from Ref. [8]. Solid and dashed lines correspond to the calculation of Arenhövel, Ref. [9], with and without MEC and IC contributions.

As an experimental indication of MEC suppression at high $Q^{2}$ in Figure 5 we 
demonstrate the $Q^{2}$ dependence of the ratios of inclusive structure functions $W_{1} / W_{2}$ for different values of $E_{p n}$. Note that $E_{p n}=0$ corresponds to the deuteron threshold where MEC should be especially enhanced. The figure clearly indicates that MEC contribution decreases with increase of $Q^{2}$ and additionally it indicates that starting at $E_{p n} \geq 50 \mathrm{MeV}$ MEC contribution becomes small at $Q^{2} \geq 1.5 \mathrm{GeV}^{2}$ region.

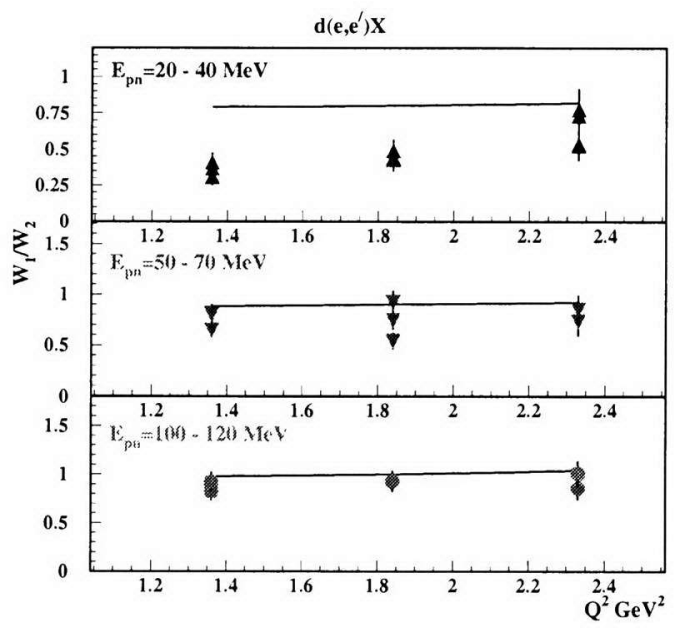

Figure 5: The $Q^{2}$ dependence of the ratios of structure functions $W_{1} / W_{2}$ from inclusive $d\left(e, e^{\prime}\right) X$ reaction. The data are from Ref.[10]. $E_{p n}=\left(W_{p n}-m_{D}-\epsilon_{d}^{\text {bound }}\right)$, where $W_{p n}^{2}=W_{\gamma^{*} D}^{2}=\left(q^{\mu}+p_{D}^{\mu}\right)^{2}$, is the C.M. energy of the proton and neutron in the final state of the reaction. Solid lines are PWIA predictions within light cone dynamics in a collinear approach [11]. 


\section{Final State Interactions}

With an increase of energy the characteristics of soft (small angle) hadronhadron interactions in the amplitude of Figure 2(b) simplify in several ways. The first major qualitative change is the emergence of a practically energy independent total cross section of hadron-hadron interactions at lab momenta $\geq 1-1.5 \mathrm{GeV} / \mathrm{c}$ (total cross sections are being constant up to momenta of $400 \mathrm{GeV} / \mathrm{c}$ ). As Figure 6
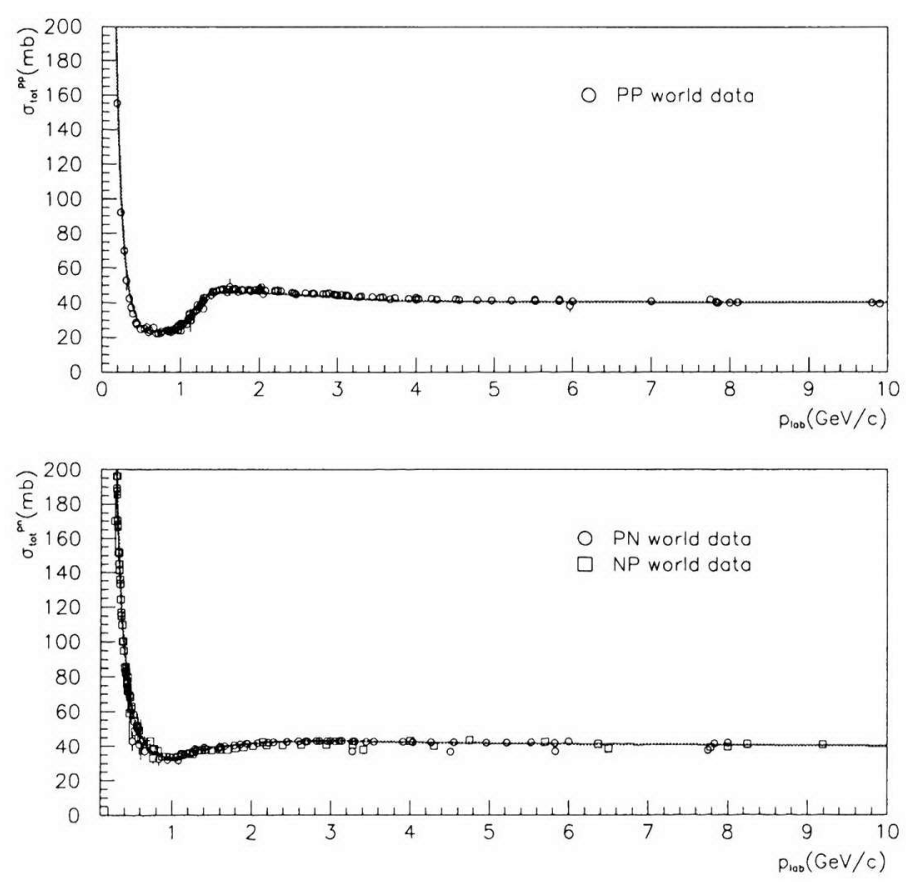

Figure 6: Proton-proton and proton-neutron total cross sections as a function of incoming proton momenta in Lab. frame. Solid lines are our fits to the world data (see Appendix D for the corresponding Fortran listings).

shows both $p p$ and $p n$ total cross sections level out and become practically energy 
independent at lab momenta greater than a few $\mathrm{GeV} / \mathrm{c}$. This simplifies tremendously the description of the final state interactions since the small angle $N N$ scattering is proportional to $\sigma_{N N}^{t o t a l}$. The additional simplification associated with the increase of energy is that at small angles the rescattering amplitude is predominantely imaginary and conserves the helicity of interacting particles. 


\section{Reduction Theorem}

Let us consider the following theorem: High energy particles propagating in the nuclear medium can not interact with the same bound nucleon a second time after interacting with another bound nucleon. In other words all those rescatterings which have a segments similar to Figure 7 are suppressed.

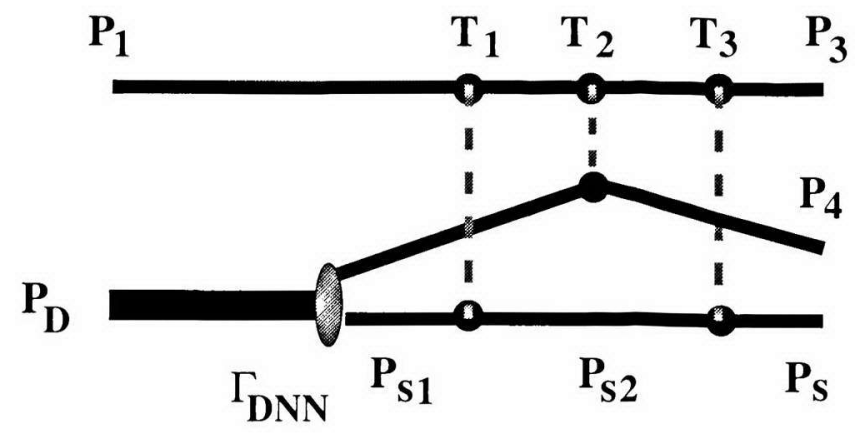

Figure 7: Diagram for hadron-deuteron scattering

We prove the above statement for the case of the amplitude of the diagram of Figure 7 . This prove can be easily generalized to all other cases. The invariant amplitude of the diagram in Figure 7 can be presented as follows:

$$
\begin{aligned}
A_{p d \rightarrow p p n}= & -\int \frac{d^{4} p_{s 1}}{i(2 \pi)^{4}} \frac{d^{4} p_{s 2}}{i(2 \pi)^{4}} \frac{T_{3}\left(p_{s}-p_{s 2}\right) T_{2}\left(p_{4}-\left(p_{D}-p_{s 1}\right)\right) T_{1}\left(p_{s 2}-p_{s 1}\right)}{D\left(p_{3}+p_{s}-p_{s 2}\right) D\left(p_{1}+p_{s 1}-p_{s 2}\right)} \\
& \times \frac{\Gamma_{D N N}\left(p_{D}-p_{s 1}, p_{s 1}\right)}{D\left(p_{s 2}\right) D\left(p_{s 1}\right) D\left(p_{D}-p_{s 1}\right)}
\end{aligned}
$$

where $D(p)=-\left(p^{2}-m^{2}+i \epsilon\right)$. We neglect the spins since they are not relevant for our discussion.

It is convenient for this section to use light-cone $\left(p_{-}, p_{+}, p_{\perp}\right)$ representation for 
four-momentum $p^{\mu}=\left(p_{0}, p_{z}, p_{\perp}\right)$, constructing the \pm components as:

$$
p_{ \pm}=p_{0} \pm p_{z}
$$

The scalar product of two four-vectors is then given by

$$
p_{1} \cdot p_{2} \equiv p_{10} p_{20}-p_{1 z} p_{2 z}-p_{1 \perp} p_{2 \perp}=\frac{1}{2} p_{1+} p_{2-}+\frac{1}{2} p_{1-} p_{2+}-p_{1 \perp} p_{2 \perp}
$$

We observe that the kinematics for the scattering corresponding to Figure 7 is such that

$$
p_{1+} \sim p_{3+} \gg m \quad \text { and } \quad p_{1-} \sim p_{3-} \ll m
$$

i.e. the propagating nucleon in the top of the Figure 7 is very energetic. Meanwhile

$$
p_{4+} \sim p_{4-} \sim p_{s+} \sim p_{s-} \sim m,
$$

i.e. recoiled nuclear system is non relativistic.

Next we introduce momenta:

$$
k_{1}=p_{s 2}-p_{s 1}, \quad k_{2}=p_{s}-p_{s 2} \quad \text { and } \quad K=k_{1}+k_{2} .
$$

This results in replacement

$$
\begin{aligned}
& p_{s 2}=p_{s}-k_{2}, \\
& p_{s 1}=p_{s 2}-k_{1}=p_{s}-k_{1}-k_{2}=p_{s}-K .
\end{aligned}
$$

Becouse $K_{0}=\left(K_{+}+K_{-}\right) / 2$ and $K_{z}=\left(K_{+}-K_{-}\right) / 2$

$$
d^{4} K=d^{2} K_{\perp} d K_{0} d K_{z}=d^{2} K_{\perp}\left|\frac{\partial\left(K_{0}, K_{z}\right)}{\partial\left(K_{+}, K_{-}\right)}\right| d K_{+} d K_{-}=\frac{1}{2} d^{2} K_{\perp} d K_{+} d K_{-}
$$


Using these definitions we obtain from eq.(4)

$$
\begin{aligned}
A_{p d \rightarrow p p n}= & \frac{1}{4} \int \frac{d^{2} K_{\perp} d^{2} k_{2 \perp}}{(2 \pi)^{4}} \frac{d K_{-} d k_{2-}}{(2 \pi)^{2}} \frac{d K_{+} d k_{2+}}{(2 \pi)^{2}} \\
& \frac{T_{3}\left(k_{2}\right) T_{2}\left(p_{4}-\left(p_{D}-p_{s}+K\right)\right) T_{1}\left(K-k_{2}\right)}{D\left(p_{3}+k_{2}\right) D\left(p_{1}+k_{2}-K\right)} \\
& \frac{\Gamma_{D N N}\left(p_{D}-p_{s}+K ; p_{s}-K\right)}{D\left(p_{s}-k_{2}\right) D\left(p_{s}-K\right) D\left(p_{D}-p_{s}+K\right)} .
\end{aligned}
$$

First we integrate over $d K_{+}$. As we consider $K_{+}$complex plane one can clearly see that poles of propagators $D\left(p_{s}-K\right)$ and $D\left(p_{1}+k_{2}-K\right)$ lay in the upper half-plane whereas $D\left(p_{D}-p_{s}+K\right)$ has pole in the lower half-plane. Therefore we can close contour of integration in the upper plane and consider only first two propagators. As we write

$$
\begin{aligned}
-D\left(p_{1}+k_{2}-K\right)= & \left(p_{1}+k_{2}-K\right)_{-}\left(p_{1}+k_{2}-K\right)_{+}- \\
& -\left(p_{1}+k_{2}-K\right)_{\perp}^{2}-m^{2}+i \varepsilon=-\left(p_{1}+k_{2}-K\right)_{-} \times \\
& \times\left[K_{+}-\left(\left(p_{1}+k_{2}\right)_{+}-\frac{\left(p_{1}+k_{2}-K\right)_{\perp}^{2}+m^{2}-i \varepsilon}{\left(p_{1}+k_{2}-K\right)_{-}}\right)\right]
\end{aligned}
$$

we observe that pole integration of this propagator will return negligible result. Indeed, application of residue theorem will replace $K_{+}$with the quantity containing $p_{1+}$. $D N N$ vertex function therefore will look like $\sim \Gamma_{D N N}\left(p_{1+}\right)$. After non-relativistic reduction of vertex function the remaining integrand will contain deutron wave function with the argument $p_{1+} \gg m$. For such a high momenta $\psi_{D} \sim 0$ and corresponding integral is strongly suppressed.

Now we turn to the last $K_{+}$pole and get

$$
-D\left(p_{s}-K\right)=\left(p_{s}-K\right)_{-}\left(p_{s}-K\right)_{+}-\left(p_{s}-K\right)_{\perp}^{2}-m^{2}+i \varepsilon=
$$




$$
=-\left(p_{s}-K\right)_{-}\left[K_{+}-\left(p_{s+}-\frac{\left(p_{s}-K\right)_{\perp}^{2}+m^{2}-i \varepsilon}{\left(p_{s}-K\right)_{-}}\right)\right]
$$

so integration over $d K_{+}$will merely leave us with the factor of $2 \pi i /\left(p_{s}-K\right)_{-}$. Using the same approach we can now integrate over $d k_{2+}$ by closing contour in the upper half plane with the single pole corresponding to propagator $D\left(p_{s}-k_{2}\right)$ (other two propagators containing $k_{2+}$ have poles in lower half-plane). As before, one can write

$$
\begin{aligned}
-D\left(p_{s}-k_{2}\right) & =\left(p_{s}-k_{2}\right)_{-}\left(p_{s}-k_{2}\right)_{+}-\left(p_{s}-k_{2}\right)_{\perp}^{2}-m^{2}+i \varepsilon= \\
& =-\left(p_{s}-k_{2}\right)_{-}\left[k_{2+}-\left(p_{s+}-\frac{\left(p_{s}-k_{2}\right)_{\perp}^{2}+m^{2}-i \varepsilon}{\left(p_{s}-k_{2}\right)_{-}}\right)\right]
\end{aligned}
$$

and effectively replace $d k_{2+}$ integration with the factor of $2 \pi i /\left(p_{s}-k_{2}\right)_{-}$.

The overall result reads:

$$
\begin{aligned}
A_{p d \rightarrow p p n}= & \frac{-1}{4} \int \frac{d^{2} K_{\perp} d^{2} k_{2 \perp}}{(2 \pi)^{4}} \frac{d K_{-} d k_{2-}}{(2 \pi)^{2}} \frac{T_{3}\left(k_{2}\right) T_{2}\left(p_{4}-\left(p_{D}-p_{s}+K\right)\right) T_{1}\left(K-k_{2}\right)}{D\left(p_{3}+k_{2}\right) D\left(p_{1}+k_{2}-K\right)} \\
& \frac{\Gamma_{D N N}\left(p_{D}-p_{s}+K ; p_{s}-K\right)}{\left(p_{s}-k_{2}\right)_{-}\left(p_{s}-K\right)_{-} D\left(p_{D}-p_{s}+K\right)}
\end{aligned}
$$

Next, to integrate over $d K_{-}$and $d k_{2-}$ one observes that in high energy limit when $p_{1+}, p_{3+} \gg m$ and $p_{1-}, p_{3-} \ll m$ the fast nucleon's propagator can be expressed as follows:

$$
\begin{aligned}
-D\left(p_{3}+k_{2}\right)= & \left(p_{3}+k_{2}\right)_{+}\left(p_{3}+k_{2}\right)_{-}-\left(p_{3}+k_{3}\right)_{\perp}^{2}-m^{2}+i \epsilon \approx p_{3+}\left(k_{2-}+i \epsilon\right) \\
-D\left(p_{1}+k_{2}-K\right)= & \left(p_{1}+k_{2}-K\right)_{+}\left(p_{1}+k_{2}-K\right)_{-}-\left(p_{1}+k_{2}-K\right)_{\perp}^{2}- \\
& -m^{2}+i \epsilon \approx p_{1+}\left(k_{2-}-K_{-}+i \epsilon\right) .
\end{aligned}
$$

Inserting these expressions into Eq.(17) one obtains:

$$
A_{p d \rightarrow p p n}=\frac{-1}{4} \int \frac{d^{2} K_{\perp} d^{2} k_{2 \perp}}{(2 \pi)^{4}} \frac{d K_{-} d k_{2-}}{(2 \pi)^{2}} \frac{T_{3}\left(k_{2}\right) T_{2}\left(p_{4}-\left(p_{D}-p_{s}+K\right)\right) T_{1}\left(K-k_{2}\right)}{p_{3+}\left(k_{2-}+i \epsilon\right) p_{1+}\left(k_{2-}-K_{-}+i \epsilon\right)}
$$




$$
\frac{\Gamma_{D N N}\left(p_{D}-p_{s}+K ; p_{s}-K\right)}{\left(p_{s}-k_{2}\right)_{-}\left(p_{s}-K\right)_{-} D\left(p_{D}-p_{s}+K\right)} \text {. }
$$

Analyzing the range of the integration over $d K_{-}$and $d k_{2}$ - we first observe that the soft rescattering amplitudes depend predominantly on $q_{\perp}$, i.e. $T_{i}(q) \sim T_{i}\left(q_{\perp}\right)$ and does not contain any singularities associated with $K_{-}$and $k_{2-}$. Other apparent singularities may come from $\left(p_{s}-k_{2}\right)_{-}=0$ and $\left(p_{s}-K\right)_{-}=0$. However according to the redefinitions of Eq.(9) they correspond to $p_{s 2-}=0$ and $p_{s 1-}=0$. The latter conditions represent a bound nucleon with infinite virtuality $p_{s 1+, 2+} \sim \frac{m^{2}}{p_{s 1-, 2-}}$ which is suppressed by wave function of bound nucleon. Therefore the structure of $d K_{-}$ and $d k_{2-}$ integrations will be defined only by two denominators of a fast propagating nucleon, i.e. by,

$$
\int \frac{d k_{2-} d K_{-}}{\left(k_{2-}+i \epsilon\right)\left(k_{2-}-K_{-}+i \epsilon\right)}=0
$$

The above integral is zero since both poles over the $k_{2-}$ are on the same side of the complex $k_{2-}$ semi-plane and one can close the contour of integration on the side where there are no singularities exist. Thus this contribution results $A_{p d \rightarrow p p n}=0$.

This result allows us to reduce potentially infinite number of rescattering diagrams to a finite set of diagrams. The only diagrams that survive are those in which a propagating fast nucleon interacts first with one target nucleon then the next one and so on, making rescatterings strictly sequential.

Another consequence of this theorem is that, if the virtuality of a bound nucleon, which is interacting with the propagating (energetic) nucleon, can be neglected, the sum of the interaction amplitudes with a given nucleon can be replaced by the in- 
variant NN scattering amplitude, $F_{N N}$ as in Figure 8 . The later can be replaced by the phenomenological NN scattering amplitude taken from the NN scattering data.

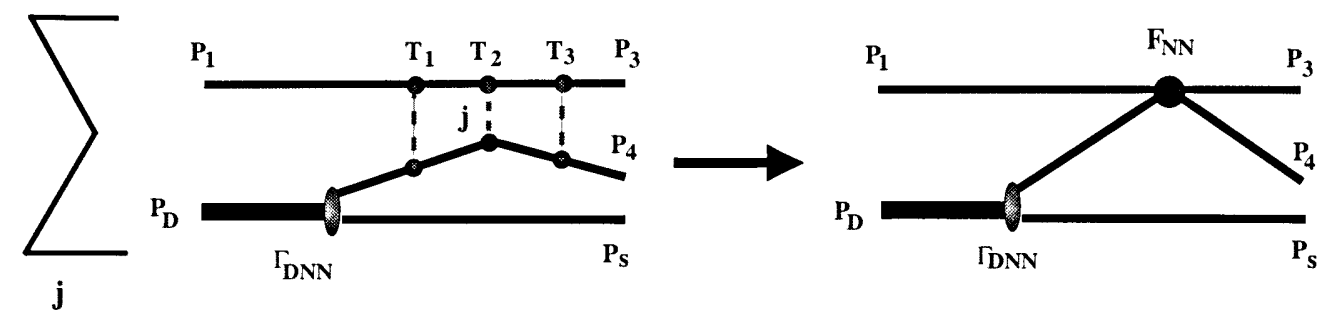

Figure 8: Reduction theorem for hadron-deuteron scattering.

Thus we will end up with the finite set of scattering diagrams for which Feynman diagram rules can be identified.

The above result represents the realization of eikonal approximation. However the major difference from the conventional semiclassical approximation is that the present approach does not require the spectator nucleons, to be a stationary scatterers[7]. Furthermore, we will refer the present approach as generalized eikonal approximation (GEA). 


\section{Feynman Diagram Rules for the Scattering}

\section{Amplitude in GEA}

In this section we will define the effective Feynman diagram rules, within GEA, for the scattering amplitude of knocked-out nucleon to undergo $n$ rescatterings off the nucleons of $(A-1)$ residual system. The case $n=0$ corresponds to the plane wave impulse approximation in which the knocked out nucleon does not interact with residual nucleus. We systematically neglect the diffractive excitation of the nucleons in the intermediate states. In soft QCD processes this is a small correction for the knock-out nucleon (projectile) energies $\leq 10 \mathrm{GeV}$. In the hard processes (that is when $Q^{2}$ - virtuality of the photon is sufficiently large $\left.\left(\geq 6-8 G e V^{2}\right)\right)$ such an approximation can not be justified even within this energy range, because of important role of quark-gluon degrees of freedom in Color Transparency phenomena. However our aim is to perform calculations in the kinematics where the color transparency phenomenon is still a small correction and intermediate hadronic states can be treated as a nucleon states.

According to the above discussion the n-fold rescattering amplitude will be represented through $n$ vertex amplitude in which the each vertex corresponds to one NN scattering - Figure 9. We can formulate the following Feynman rules of calculation of the diagram of Figure 9 (see also Ref.[6]).

- We assigns the vertex functions $\Gamma_{A}\left(p_{1}, \ldots, p_{A}\right)$ and $\Gamma_{A-1}^{\dagger}\left(p_{2}^{\prime}, \ldots, p_{A}\right)$ to describe the transitions between "nucleus $A^{\prime \prime}$ to "A nucleons" with momenta $\left\{p_{n}\right\},\left\{p_{n}^{\prime}\right\}$ 


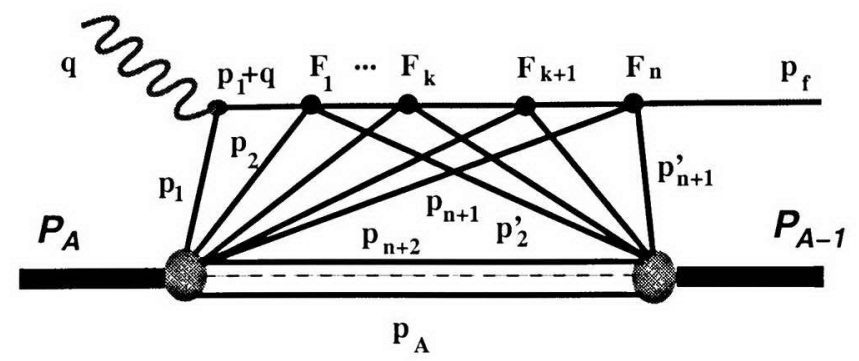

Figure 9: Diagram for n-fold rescattering.

and " $(A-1)$ nucleons" with momenta $\left\{p_{n}^{\prime}\right\}$ to " $(A-1)$ nucleon final state" respectively.

- For $\gamma^{*} N$ interaction we assign vertex, $\Gamma_{\gamma^{*} N}^{\mu}$.

- For each $N N$ interaction we assign the vertex function $F_{k}^{N N}\left(p_{k+1}, p_{k+1}^{\prime}\right)$. This vertex function are related to the amplitude of $N N$ scattering as follows:

$$
\bar{u}\left(p_{3}\right) \bar{u}\left(p_{4}\right) F^{N N} u\left(p_{1}\right) u\left(p_{2}\right)=\sqrt{s\left(s-4 m^{2}\right)} f^{N N}\left(p_{3}, p_{1}\right) \delta_{\lambda, \lambda^{\prime}} \approx s f^{N N}\left(p_{3}, p_{1}\right) \delta_{\lambda, \lambda^{\prime}},
$$

where $s$ is the total invariant energy of two interacting nucleons with momenta $p_{1}$ and $p_{2}$ and

$$
f^{N N}=\sigma_{t o t}^{N N}(i+\alpha) e^{-\frac{B}{2}\left(p_{3}-p_{1}\right)_{\perp}^{2}}
$$

where $\sigma_{t o t}^{N N}, \alpha$ and $B$ are known experimentally from $N N$ scattering data. The vertex functions are accompanied with $\delta$-function of energy-momentum conservation.

- For each intermediate nucleon with four momentum $p$ we assign propagator 
$D(p)^{-1}=-(\hat{p}-m+i \epsilon)^{-1}$. Following to Ref.[12] we choose the "minus" sign for the nucleon propagators to simplify the calculation of the overall sign of the scattering amplitude.

- The factor $n !(A-n-1)$ ! accounts for the combinatorics of $n$-rescatterings and $(A-n-1)$ spectator nucleons.

- For each closed contour one gets the factor $\frac{1}{i(2 \pi)^{4}}$ with no additional sign.

Using above defined rules for the scattering amplitude of Figure 9 one obtains:

$$
\begin{aligned}
& F_{A, A-1}^{(n)}\left(q, p_{f}\right)=\sum_{h} \frac{1}{n !(A-n-1) !} \prod_{i=1}^{A} \prod_{j=2}^{A} \int d^{4} p_{i} d^{4} p_{j}^{\prime} \frac{1}{\left[i(2 \pi)^{4}\right]^{A-2+n}} \\
& \delta^{4}\left(\sum_{i=1}^{A} p_{i}-\mathcal{P}_{A}\right) \delta^{4}\left(\sum_{j=2}^{A} p_{j}^{\prime}-\mathcal{P}_{A-1}\right) \prod_{m=n+2}^{A} \delta^{4}\left(p_{m}-p_{m}^{\prime}\right) \times \\
& \frac{\Gamma_{A-1}\left(p_{2}^{\prime}, . ., p_{n+1}^{\prime}, p_{n+2}, . ., p_{A}\right)}{D\left(p_{2}^{\prime}\right) . . D\left(p_{n+1}^{\prime}\right)} \frac{f_{1}^{N N}\left(p_{2}, p_{2}^{\prime}\right) . . f_{n}^{N N}\left(p_{n+2}, p_{n+2}^{\prime}\right)}{D\left(l_{1}\right) . . D\left(l_{k}\right) . . D\left(l_{n-1}\right)} \frac{\Gamma_{\gamma^{*} N}^{\mu(h)}\left(Q^{2}\right)}{D\left(p_{1}+q\right)} \times \\
& \frac{\Gamma_{A}\left(p_{1}, \ldots, p_{A}\right)}{D\left(p_{1}\right) D\left(p_{2}\right) . . D\left(p_{n+1}\right) D\left(p_{n+2}\right) . . D\left(p_{A}\right)}
\end{aligned}
$$

where, for the sake of simplicity, we neglect the spin dependent indices. Here $\mathcal{P}_{A}$ and $\mathcal{P}_{A-1}$ are the four momenta of the target nucleus, and final $(A-1)$ system, $p_{j}$ and $p_{j}^{\prime}$ are nucleon momenta in the nucleus $A$ and residual $(A-1)$ system respectively. $\sum_{h}$ in Eq.(23) goes over virtual photon interactions with different nucleons, where $\Gamma_{\gamma^{*} N}^{\mu(h)}$ are electromagnetic vertices. $-D\left(p_{k}\right)^{-1}$ is the propagator of a nucleon with momentum $p_{k}$ and $-D\left(l_{k}\right)^{-1}$ is the propagator of the struck nucleon in the intermediate state, with momentum $l_{k}=q+p_{1}+\sum_{i=2}^{k}\left(p_{i}-p_{i}^{\prime}\right)$ between $k-1$-th and $k$-th rescatterings. The intermediate spectator states in the diagram of Figure 9 are expressed in terms 
of nucleons but not nuclear fragments because the closure over various nuclear excitations in the intermediate state is used. The possibility to use closure is related to the fact that the typical scale characteristic for high energy phenomena is significantly larger then the energy scale of nuclear excitations.

After evaluation of the intermediate state nucleon propagators, the covariant amplitude will be reduced to a set of time ordered non covariant diagrams. This will help to establish the correspondence between the nuclear vertex functions and the nuclear wave functions. Particularly in the nonrelativistic limit the momentum space wave function is defined through the vertex function as follows[13]:

$$
\psi_{A}\left(p_{1}, p_{2}, \ldots p_{A}\right)=\frac{1}{\left(\sqrt{(2 \pi)^{3} 2 m}\right)^{A-1}} \frac{\Gamma_{A}\left(p_{1}, p_{2}, \ldots p_{A}\right)}{D\left(p_{1}\right)}
$$

where wave functions are normalized as: $\int\left|\psi_{A}\left(p_{1}, p_{2}, \ldots p_{A}\right)\right|^{2} d^{3} p_{1} d^{2} p_{2} . . d^{3} p_{A}=1$. 


\section{Scattering Amplitudes for ${ }^{3} \mathrm{He}\left(e, e^{\prime} N N\right) N$}

\section{Reaction}

\subsection{Plane Wave Impulse Approximation and Pair Distortion Amplitudes}

For the case when no final state rescatterings of leading nucleon occur straightforward application of Feynman rules for $n=0$ term from previous section yields:

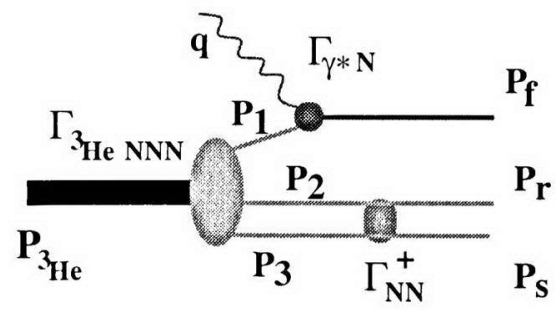

Figure 10: Impulse Approximation Diagram.

$$
\begin{aligned}
A_{0}^{\mu}+A_{0 s}^{\mu} & =-\int \frac{d^{4} p_{2}}{i(2 \pi)^{4}} \bar{u}\left(p_{s}\right) \bar{u}\left(p_{r}\right) \bar{u}\left(p_{f}\right) \cdot \Gamma_{N N}^{+}\left(p_{2}, p_{3}\right) \cdot \Gamma_{\gamma^{*} N}^{\mu} \cdot \frac{\left[\hat{p}_{3}+m\right]}{p_{3}^{2}-m^{2}+i \varepsilon} \times \\
& \times \frac{\left[\hat{p}_{2}+m\right]}{p_{2}^{2}-m^{2}+i \varepsilon} \cdot \frac{\left[\hat{p}_{1}+m\right]}{p_{1}^{2}-m^{2}+i \varepsilon} \cdot \Gamma_{{ }^{3} \mathrm{HeNNN}}\left(p_{1}, p_{2}, p_{3}\right) \chi^{{ }^{3} \mathrm{He}}
\end{aligned}
$$

Here $A_{0}^{\mu}$ is refering to impulse approximation amplitude and $A_{0 s}^{\mu}$ stands for coupling of spectator pair. $\hat{p} \equiv p_{\mu} \gamma^{\mu}$ and $\Gamma_{N N}^{+}\left(p_{2}, p_{3}\right)$ describes, in the most general case, a transition of two $(N N)$ intermediate nucleon to final continuum or bound twonucleon state. Since we are considering specific ${ }^{3} \mathrm{He}\left(e, e^{\prime} N N\right) N$ break-down reaction this vertex function in our calculations assosiated with the free(continuum) $N N$ state 
only. The spin function of ${ }^{3} \mathrm{He}$ nucleus is denoted by $\chi^{3} \mathrm{He} . \Gamma_{{ }^{3} \mathrm{HeNNN}}$ corresponds to covariant ${ }^{3} \mathrm{He} \rightarrow N N N$ transition vertex and $\Gamma_{\gamma^{*} N}^{\mu}$ is a covariant electromagnetic vertex of the $\gamma^{*} N_{\text {bound }} \rightarrow N$ transition. Latter has the form:

$$
\Gamma_{\gamma^{*} N}^{\mu} \sim A \cdot \gamma^{\mu}+B \cdot \sigma^{\mu \nu} q^{\nu}
$$

where $q^{\nu}$, as usually, is a momentum transferred to nucleus, and $A$ and $B$ are functions of scalar combinations of $q^{\mu}, p_{f}^{\mu}$ and $\left(p_{3_{3} \mathrm{He}}-p_{r}-p_{s}\right)^{\mu}$. It's worth to note that since both $\Gamma_{{ }^{3} \mathrm{HeNNN}}$ and $\Gamma_{\gamma^{*} N}^{\mu}$ are relativistic covariant vertices therefore in the time ordered expansion they contain both impulse approximation and vacuum fluctuation diagrams. This implies that $\Gamma_{\gamma^{*} N}^{\mu}$ may represent, for example, $\gamma^{*} \rightarrow \tilde{N} N$ and $\Gamma^{{ }^{3} \mathrm{HeNNN}}$ also describe the processes like $\bar{N}^{3} \mathrm{He} \rightarrow N N$. However, if we restrict by the kinematics defined in eq.(2), then in the set of noncovariant diagrams, comprising the covariant diagram of Fig. 10, one can neglect the diagrams which correspond to the vacuum fluctuations. Moreover, since we are interested in target nucleon momenta $\vec{p}_{m}^{2} \ll m^{2}$ one can neglect off-shell effects in the nucleon spinors and use the closure for the nucleons in the intermediate states:

$$
\hat{p}_{1,2,3}+m=\sum_{s} u\left(p_{1,2,3}, s\right) \bar{u}\left(p_{1,2,3}, s\right)
$$

(we have adopted normalization $\bar{u}(p, s) u(p, s)=2 m)$. Thus we have neglected nonnucleon degrees of freedom in a nucleus. Overall in discussed kinematics the relativistic effects in the nuclear wave function are a small correction and the impulse approximation(IA) can be calculated via nonrelativistic reduction of the covariant nuclear vertices in eq.(25). Such a reduction corresponds to taking the residue over 
$d p_{2}^{0}$, at the nearest nucleon pole in the spectator nucleon propagator. The restriction by the nearest pole in the nucleon propagators follows from the observation that this is the only pole not associated with $N \bar{N}$ production in our kinematics, where nuclear excitations are small as compared to the scale of energies characteristic for the nucleon excitations. After such integration one is left with the time ordered diagram corresponding to the IA(or rather distorted IA), where the virtual photon knocks out the target nucleon with momentum $p_{1}$.

Returning to eq.(25) we replace projection operators by closure expansion of eq.(27):

$$
\begin{aligned}
A_{0}^{\mu}+A_{0 s}^{\mu} & =-\sum_{s_{1} s_{2} s_{3}} \int \frac{d p_{2}^{0} d^{3} p_{2}}{i(2 \pi)^{4}} \bar{u}\left(p_{s}\right) \bar{u}\left(p_{r}\right) \bar{u}\left(p_{f}\right) \cdot \Gamma_{N N}^{+}\left(p_{2}, p_{3}\right) \cdot \Gamma_{\gamma^{*} N}^{\mu} \times \\
& \times \frac{u\left(p_{3}, s_{3}\right) \bar{u}\left(p_{3}, s_{3}\right)}{p_{3}^{2}-m^{2}+i \varepsilon} \cdot \frac{u\left(p_{2}, s_{2}\right) \bar{u}\left(p_{2}, s_{2}\right)}{\left(p_{2}^{0}\right)^{2}-\left|\mathbf{p}_{\mathbf{2}}\right|^{2}-m^{2}+i \varepsilon} \times \\
& \times \frac{u\left(p_{1}, s_{1}\right) \bar{u}\left(p_{1}, s_{1}\right)}{p_{1}^{2}-m^{2}+i \varepsilon} \cdot \Gamma_{{ } \mathrm{HeNNN}}\left(p_{1}, p_{2}, p_{3}\right) \chi^{3 \mathrm{He}} .
\end{aligned}
$$

In order to integrate over $d p_{2}^{0}$ by contour method we note that corresponding poles are located at $\pm \sqrt{\left|\mathbf{p}_{\mathbf{2}}\right|^{2}+m^{2}}$. Adding $\mp i \varepsilon$ one can displace the poles from the real axis and by closing the contour in the upper complex half-plane easily compute the following improper integral:

$$
\int_{-\infty}^{\infty} \frac{d p_{2}^{0}}{\left(p_{2}^{0}-\sqrt{\left|\mathbf{p}_{\mathbf{2}}\right|^{2}+m^{2}}+i \varepsilon\right)\left(p_{2}^{0}+\sqrt{\left|\mathbf{p}_{\mathbf{2}}\right|^{2}+m^{2}}-i \varepsilon\right)}=\frac{2 \pi i}{-2 \sqrt{\left|\mathbf{p}_{\mathbf{2}}\right|^{2}+m^{2}}}
$$

Since we employ non-relativistic treatment of the spectator pair $\left(\left|\mathbf{p}_{\mathbf{2}}\right|^{2} / m^{2} \ll 1\right)$ 
this reduces to

$$
\frac{2 \pi i}{-2 \sqrt{\left|\mathbf{p}_{2}\right|^{2}+m^{2}}} \approx-\frac{2 \pi i}{2 m} \text {. }
$$

Keeping in mind that every argument $p_{2}^{0}$ is now assumed to be replaced with the residue $-\sqrt{\left|\mathbf{p}_{\mathbf{2}}\right|^{2}+m^{2}}$ we rewrite eq.(28) as

$$
\begin{aligned}
A_{0}^{\mu}+A_{0 s}^{\mu} & =\sum_{s_{1} s_{2} s_{3}} \int \frac{d^{3} p_{2}}{2 m(2 \pi)^{3}} \frac{\bar{u}\left(p_{r}\right) \bar{u}\left(p_{s}\right) \Gamma_{N N}^{+}\left(p_{2}, p_{3}\right) u\left(p_{3}, s_{3}\right) u\left(p_{2}, s_{2}\right)}{p_{3}^{2}-m^{2}+i \varepsilon} \times \\
& \times \bar{u}\left(p_{f}\right) \Gamma_{\gamma^{*} N}^{\mu} u\left(p_{1}, s_{1}\right) \times \\
& \times \frac{\bar{u}\left(p_{1}, s_{1}\right) \bar{u}\left(p_{2}, s_{2}\right) \bar{u}\left(p_{3}, s_{3}\right) \Gamma_{3} \mathrm{HeNNN}_{N}\left(p_{1}, p_{2}, p_{3}\right) \chi^{3} \mathrm{He}}{p_{1}^{2}-m^{2}+i \varepsilon} .
\end{aligned}
$$

Now we are going to apply the non-relativistic reduction of the initial $\left(\Gamma_{3} \mathrm{HeNNN}\right)$ and residual $\left(\Gamma_{N N}^{+}\right)$systems' vertex functions in order to substitute them with the corresponding non-relativistic momentum space nuclear wave functions. The general formula for the system of $A$ nucleons is given by

$$
\begin{aligned}
\Psi_{A}^{S_{A}}\left(p_{1}, s_{1} ; p_{2}, s_{2} ; \ldots ; p_{A}, s_{A}\right)= & \frac{1}{\left(\sqrt{(2 \pi)^{3} 2 m}\right)^{A-1}} \\
& \frac{\bar{u}\left(p_{1}, s_{1}\right) \ldots \bar{u}\left(p_{A}, s_{A}\right) \Gamma_{A}\left(p_{1}, p_{2}, \ldots, p_{A}\right) \chi^{A}}{-\left(p_{1}^{2}-m^{2}+i \varepsilon\right)}
\end{aligned}
$$

where nucleon propagator by convention chosed to carry "minus" sign. The wave function is normalized as:

$$
\int\left|\Psi_{A}^{S_{A}}\left(p_{1}, p_{2}, \ldots, p_{A}\right)\right|^{2} \delta^{3}\left(\sum_{i=1}^{A} p_{i}-P_{A}\right) d^{3} p_{1} d^{2} p_{2} \ldots d^{3} p_{A}=N
$$

where $\mathrm{N}=1$ for bound states and $N=\prod_{i=1}^{A} \delta^{3}\left(p_{i}-p_{i}^{\prime}\right)$ for $A$ body continuum state. We note, that to apply $\bar{u} u=2 m$ relativistic normalization for the spinors the 
${\sqrt{(2 \pi)^{2} 2 m}}^{-1}$ phase factor should be associated with the plane wave single nucleon wave function.

Using eq.(32) for the case of bound system of two proton and one neutron the last term in integrand of eq.(31) can be replaced by:

$$
\begin{aligned}
& -\frac{\bar{u}\left(p_{1}, s_{1}\right) \bar{u}\left(p_{2}, s_{2}\right) \bar{u}\left(p_{3}, s_{3}\right) \Gamma_{3 \mathrm{HeNNN}}\left(p_{1}, p_{2}, p_{3}\right) \chi^{3 \mathrm{He}}}{p_{1}^{2}-m^{2}+i \varepsilon}=(2 \pi)^{3}(2 m) \times \\
& \times \Psi_{3 \mathrm{He}}^{S_{3 \mathrm{He}}}\left(p_{1}, s_{1} ; p_{2}, s_{2} ; p_{3}, s_{3}\right) .
\end{aligned}
$$

The ground-state wave function of ${ }^{3} \mathrm{He}$, introduced this way, is chosen to be normalized as

$$
\sum_{s_{1}, s_{2}, s_{3}} \int d^{3} p_{1} d^{3} p_{2} d^{3} p_{3}\left|\Psi_{{ }_{\mathrm{He}}}^{S_{3_{\mathrm{He}}}}\left(p_{1}, s_{1} ; p_{2}, s_{2} ; p_{3}, s_{3}\right)\right|^{2} \delta^{3}\left(p_{1}+p_{2}+p_{3}\right)=1
$$

Using the similar approach, the unbound two-nucleon state, described by the first term in eq.(31), can be connected with final state continuum wave function: $\Gamma_{N N}^{+} \rightarrow \Psi_{N N}^{*} \equiv\left(\Psi_{\mathbf{k}}^{(-)}(\mathbf{q})\right)^{*}=\Psi_{\mathbf{k}}^{(+)}(\mathbf{q})$. The detailed discussion of this function carried out in the Appendix A. It is shown there that asymptotic form of $\Psi^{(+)}$in coordinate representation is given by plane wave $e^{i \mathbf{k r}}$ (delta-function in momentum representation) and diverging ("+") spherical wave. In scattering theory this function describes a particle of known energy $k^{2} / 2 m_{\text {red }}$ scattered in field $U(r)$ of fixed force center. Such picture is equivalent to the considered coupling of two non-relativistic residual nucleons. $m_{\text {red }}$ is a reduced mass of the system whereas $\mathbf{q}$ and $\mathbf{k}$ are relative momenta of incident and outgoing pairs, respectively. If we neglect mass difference between proton and neutron then the reduced mass is just $m_{\text {red }}=m / 2$, and we have 
also $\mathbf{q}=\left(\mathbf{p}_{2}-\mathbf{p}_{3}\right) / 2, \mathbf{k}=\left(\mathbf{p}_{r}-\mathbf{p}_{s}\right) / 2$. It is convenient, therefore, to refer to $\Psi_{\mathbf{k}}^{(+)}(\mathbf{q})$ as $\Psi_{\mathbf{k}_{r s}}^{(+)}\left(\left(\mathbf{p}_{2}-\mathbf{p}_{3}\right) / 2\right)$. With this notation we have:

$$
-\frac{\bar{u}\left(p_{r}\right) \bar{u}\left(p_{s}\right) \Gamma_{N N}^{+}\left(p_{2}, p_{3}\right) u\left(p_{3}, s_{3}\right) u\left(p_{2}, s_{2}\right)}{p_{3}^{2}-m^{2}+i \varepsilon}=2 E_{r} 2 E_{s} \Psi_{\mathbf{k}_{r s}}^{(+)}\left(\frac{p_{2}-p_{3}}{2}\right) .
$$

Finally we can introduce electromagnetic current as

$$
j^{\mu}\left(p_{f}, s_{f}, p_{m}, s_{m}\right)=\bar{u}\left(p_{f}, s_{f}\right) \Gamma_{\gamma^{*} N}^{\mu} u\left(p_{m}, s_{m}\right)
$$

If we replace all three terms under the integral sign in eq.(28) with the obtained expressions we will get:

$$
A_{0}^{\mu}+A_{0 s}^{\mu}=F \sum_{\text {spins }} \int d^{3} p_{2} \Psi_{\mathbf{k}_{r s}}^{(+)}\left(\frac{p_{2}-p_{3}}{2}\right) j^{\mu}\left(p_{m}+q, p_{m}\right) \Psi_{3 \mathrm{He}}\left(p_{m} ; p_{2} ; p_{3}\right)
$$

where we have substituted $\mathbf{p}_{1}=\mathbf{p}_{f}-\mathbf{q} \equiv \mathbf{p}_{m}$ and $\mathbf{p}_{f}=\mathbf{p}_{m}+\mathbf{q}$. We also set $F \equiv 2 E_{r} 2 E_{s}$.

This is the conventional formula for distorted IA amplitude (the spin coordinates are suppressed for simplicity).

Observing that $p_{2}+p_{3}=p_{r}+p_{s}$ it is handy to define a new variable

$$
\mathbf{k}_{23}=\frac{\mathbf{p}_{2}-\mathbf{p}_{3}}{2}=\mathbf{p}_{2}-\frac{\mathbf{p}_{r}+\mathbf{p}_{s}}{2}
$$

so it replaces $\mathbf{p}_{2}=\mathbf{k}_{23}+\left(\mathbf{p}_{r}+\mathbf{p}_{s}\right) / 2, \mathbf{p}_{3}=-\mathbf{k}_{23}+\left(\mathbf{p}_{r}+\mathbf{p}_{s}\right) / 2$ and we arrive at

$$
\begin{aligned}
A_{0}^{\mu}+A_{0 s}^{\mu} & =F \sum_{\text {spins }} \int d^{3} k_{23} \Psi_{\mathbf{k}_{r s}}^{(+)}\left(k_{23}\right) j^{\mu}\left(p_{m}+q, p_{m}\right) \times \\
& \times \Psi_{3_{\mathrm{He}}}\left(p_{m} ; k_{23}+\frac{p_{r}+p_{s}}{2} ;-k_{23}+\frac{p_{r}+p_{s}}{2}\right) .
\end{aligned}
$$


Accurate non-relativistic theory suggests that $\Psi_{\mathbf{k}_{r s}}^{(+)}\left(k_{23}\right)$ can be represented in the following way (see Appendix A):

$$
\Psi_{\mathbf{k}_{r s}}^{(+)}\left(\mathbf{k}_{23}\right)=(2 \pi)^{3} \delta^{3}\left(\mathbf{k}_{23}-\mathbf{k}_{r s}\right)+4 \pi \frac{f_{N N}^{\text {off shell }}\left(\mathbf{k}_{r s}, \mathbf{k}_{23}\right)}{k_{23}^{2}-k_{r s}^{2}-i \varepsilon}
$$

where $\mathbf{k}_{r s}=\left(\mathbf{p}_{r}-\mathbf{p}_{s}\right) / 2$, and $f_{N N}^{\text {off shell }}$ is a non-relativistic amplitude for scattering of two bound nucleon. Two terms of eq.(41) characterize two distinctive dynamics of production of recoil(spectator) $N N$ state. If only first term of eq.(41) is kept in 40 this will correspond to the approximation in which all three final nucleons propagate as a plane wave (PWIA approximation). The second term describes reinteraction between the pair of the slow nucleons which distorts the plane wave of outgoing recoil nucleons.

Thus, the integration over the three-dimensional delta-function provides us with Plane Wave Impulse Approximation amplitude (Born term), which is written in the following form:

$$
A_{0}^{\mu}=(2 \pi)^{3} F \sum_{\text {spins }} j^{\mu}\left(p_{m}+q, p_{m}\right) \Psi_{{ }^{H e}}\left(p_{m} ; p_{r} ; p_{s}\right)
$$

Note, that we were able to express all the arguments through observables available from the ${ }^{3} \mathrm{He}\left(e, e^{\prime} N N\right) N$ experiment.

So much for PWIA term. For pair distortion amplitude the expression we left with has the form of

$$
\begin{aligned}
A_{0 s}^{\mu} & =4 \pi F \sum_{\text {spins }} \int d^{3} k_{23} \frac{f_{N N}^{\text {off shell }}\left(\mathbf{k}_{r s}, \mathbf{k}_{23}\right)}{k_{23}^{2}-k_{r s}^{2}-i \varepsilon} \cdot j^{\mu}\left(p_{m}+q, p_{m}\right) \times \\
& \times \quad \Psi_{3 \mathrm{He}}\left(p_{m} ; k_{23}-p_{m} / 2 ;-k_{23}-p_{m} / 2\right),
\end{aligned}
$$


where we have used $-\left(\mathbf{p}_{r}+\mathbf{p}_{s}\right)=\mathbf{p}_{f}-\mathbf{q} \equiv \mathbf{p}_{m}$ with $\mathbf{p}_{m}$ being a missing momentum. To proceed further we recall the identity which holds under the sign of integral:

$$
\frac{1}{\alpha-\alpha_{c}+i \varepsilon}=\mathrm{P} \cdot \mathrm{V} \cdot \frac{1}{\alpha-\alpha_{c}}-i \pi \delta\left(\alpha-\alpha_{c}\right),
$$

where P.V., as always, denotes Cauchy principal value integral. With the help of this we can replace the denominator in eq.(43) as follows:

$$
\frac{1}{\mathbf{k}_{23}^{2}-\mathbf{k}_{r s}^{2}-i \varepsilon}=\underbrace{i \pi \delta\left(\mathbf{k}_{23}^{2}-\mathbf{k}_{r s}^{2}\right)}_{\text {pole term }}+\underbrace{\mathrm{P} . \mathrm{V} \cdot \frac{1}{\mathbf{k}_{23}^{2}-\mathbf{k}_{r s}^{2}}}_{\text {P.V.term }} .
$$

We will restrict our calculations with the pole term only. This is corresponding to the scattering problem of unbound nucleons. Within the framework of considered kinematics, contribution of P.V. term plays the role of a small correction. Rewriting $d^{3} k_{23}=1 / 2\left|\mathbf{k}_{23}\right| d k_{23}^{2} d \Omega_{23}$ one has

$$
\begin{aligned}
A_{0 s}^{\mu(\text { pole })} & =2 i \pi^{2} F \sum_{\text {spins }} \int\left|\mathbf{k}_{23}\right| d k_{23}^{2} d \Omega_{23} \delta\left(\mathbf{k}_{23}^{2}-\mathbf{k}_{r s}^{2}\right) f_{N N}^{\text {on shell }}\left(\mathbf{k}_{r s}, \mathbf{k}_{23}\right) \times \\
& \times j^{\mu}\left(p_{m}+q, p_{m}\right) \cdot \Psi_{3_{\mathrm{He}}}\left(p_{m} ; k_{23}-\frac{p_{m}}{2} ;-k_{23}-\frac{p_{m}}{2}\right)
\end{aligned}
$$

or

$$
\begin{aligned}
A_{0 s}^{\mu(\text { pole })} & =\pi^{2} F \sum_{\text {spins }} \int d \Omega_{23} 2 i\left|\mathbf{k}_{r s}\right| f_{N N}^{\text {on shell }}\left(\mathbf{k}_{r s}, \mathbf{k}_{r s}^{\prime}\right) \times \\
& \times j^{\mu}\left(p_{m}+q, p_{m}\right) \cdot \Psi_{{ }^{H e}}\left(p_{m} ; k_{r s}^{\prime}-\frac{p_{m}}{2} ;-k_{r s}^{\prime}-\frac{p_{m}}{2}\right),
\end{aligned}
$$

where $\mathbf{k}_{r s}^{\prime}$ is a vector which has absolute value of $\mathbf{k}_{r s}$ but points in the direction of $\mathbf{k}_{23}$.

Thus, the final expression for pair distortion amplitude can be put in the following 
form:

$$
\begin{aligned}
A_{0 s}^{\mu(\text { pole })} & =\pi^{2} F \sum_{\text {spins }} j^{\mu}\left(p_{m}+q, p_{m}\right) \int d \Omega_{23} \widetilde{f}_{N N}^{\text {on shell }}\left(\mathbf{k}_{r s}, \mathbf{k}_{r s}^{\prime}\right) \times \\
& \times \Psi_{{ }^{H} \mathrm{He}}\left(p_{m} ; k_{r s}^{\prime}-\frac{p_{m}}{2} ;-k_{r s}^{\prime}-\frac{p_{m}}{2}\right),
\end{aligned}
$$

where $N N$ scattering amplitude can be expressed through partial waves phase shifts $\delta_{l}$ using the well-known expansion

$$
\begin{aligned}
\tilde{f}_{N N}^{\text {on shell }}\left(\mathbf{k}_{r s}, \mathbf{k}_{r s}^{\prime}\right) & \equiv 2 i\left|\mathbf{k}_{r s}\right| \cdot f_{N N}^{\text {on shell }}\left(\mathbf{k}_{r s}, \mathbf{k}_{r s}^{\prime}\right)= \\
& =\sum_{l=0}^{\infty}(2 l+1)\left(\exp \left(2 i \delta_{l}\right)-1\right) P_{l}\left(\frac{\mathbf{k}_{r s}}{\left|\mathbf{k}_{r s}\right|} \cdot \frac{\mathbf{k}_{r s}^{\prime}}{\left|\mathbf{k}_{r s}^{\prime}\right|}\right)
\end{aligned}
$$

In our numerical calculations we have used Fortran90 code NNSOLA.f (solution SP03), courtesy of Virginia Tech Partial-Wave Analysis Group (SAID), which enables to compute polarized $\tilde{f}_{N N}$ amplitude based on parametrization of world experimental data on proton-proton and neutron-proton scatterings. 


\subsection{Single Rescattering Amplitudes}

The diagrams in Fig.11(a) and 11(b) describe the process where the fast $\left(p_{f}\right)$ knocked out nucleon rescatters off one of the spectator nucleons. The general expression with suppressed spinors for the amplitude corresponding to the diagram in Fig. 11(a) is given by $n=1$ term of eq.(23) as follows:

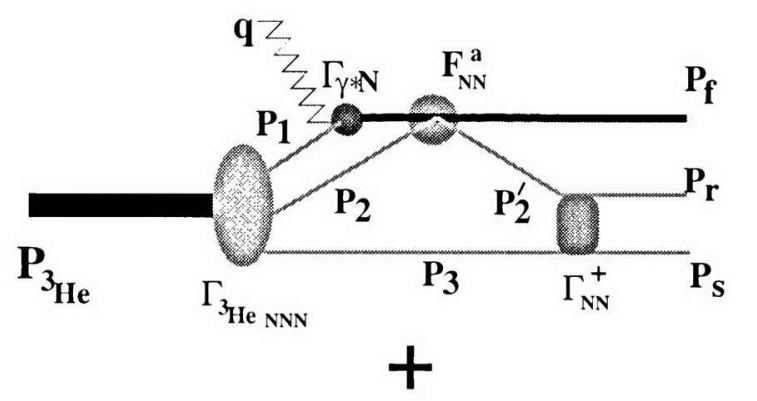

(a)

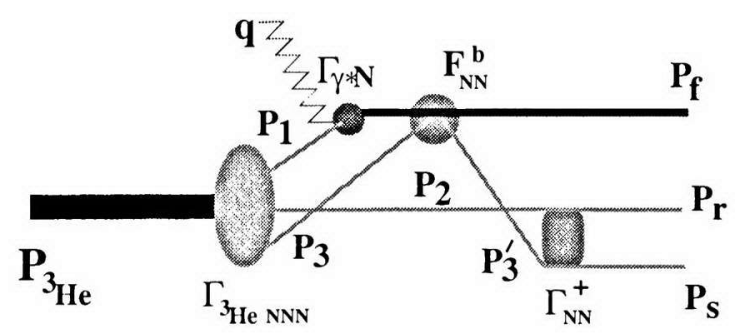

(b)

Figure 11: Single Rescattering Diagrams.

$$
A_{1 \mathrm{a}}^{\mu}=\int \frac{d^{4} p_{2}}{i(2 \pi)^{4}} \frac{d^{4} p_{3}}{i(2 \pi)^{4}} \frac{\Gamma_{N N}^{+}\left(p_{2}^{\prime}, p_{3}\right)}{D\left(p_{2}^{\prime}\right)} \cdot \frac{F_{N N}^{\mathrm{a}}\left(p_{2}^{\prime}-p_{2}\right)}{D\left(p_{1}+q\right)} \cdot \Gamma_{\gamma^{*} N}^{\mu} \frac{\Gamma_{3_{\mathrm{He} N N N}}\left(p_{1}, p_{2}, p_{3}\right)}{D\left(p_{1}\right) D\left(p_{2}\right) D\left(p_{3}\right)}
$$

where, as before, $D^{-1}(p)=-\left(p^{2}-m^{2}+i \varepsilon\right)^{-1}$ is the propagator of nucleon with the momentum $p$ together with the "minus" sign, according to the convention adopted earlier. We assume summation over initial spin values of three-body system. The 
$N N$ interaction vertex function accountable for Fig.11(a) rescattering denoted as $F_{N N}^{\mathrm{a}}$. Energy-momentum conservation provides us with obvious relations for the momenta in the intermediate states

$$
p_{1}=P_{3_{\mathrm{He}}}-p_{2}-p_{3}, \quad p_{2}^{\prime}=p_{r}+p_{s}-p_{3}
$$

Rewriting original eq.(50) in the explicit spinor-dependent form we obtain

$$
\begin{aligned}
A_{1 \mathrm{a}}^{\mu} & =-\int \frac{d^{4} p_{2}}{i(2 \pi)^{4}} \frac{d^{4} p_{3}}{i(2 \pi)^{4}} \bar{u}\left(p_{s}\right) \bar{u}\left(p_{r}\right) \bar{u}\left(p_{f}\right) \frac{\Gamma_{N N}^{+}\left(p_{2}^{\prime}, p_{3}\right)\left[\hat{p}_{2}^{\prime}+m\right]}{p_{2}^{\prime 2}-m^{2}+i \varepsilon} \times \\
& \times \frac{F_{N N}^{\mathrm{a}}\left(p_{2}^{\prime}-p_{2}\right)\left[\hat{p}_{1}+\hat{q}+m\right]}{\left(p_{1}+q\right)^{2}-m^{2}+i \varepsilon} \cdot \Gamma_{\gamma^{*} N}^{\mu} \cdot \frac{\left[\hat{p}_{3}+m\right]}{p_{3}^{2}-m^{2}+i \varepsilon} \times \\
& \times \frac{\left[\hat{p}_{2}+m\right]}{p_{2}^{2}-m^{2}+i \varepsilon} \cdot \frac{\left[\hat{p}_{1}+m\right]}{p_{1}^{2}-m^{2}+i \varepsilon} \cdot \Gamma_{{ }^{\mathrm{He} N N N}}\left(p_{1}, p_{2}, p_{3}\right) \chi^{{ }^{3} \mathrm{He}}
\end{aligned}
$$

Using the same arguments as in previous section we neglect vacuum fluctuation diagrams. Taking advantage of the considered kinematics we also neglect off-shell effects in the nuclear spinors. This enables us to use closure expansion of eq.(27) and rewrite previous equation as

$$
\begin{aligned}
A_{1 \mathrm{a}}^{\mu} & =-\sum_{\mathrm{spins}} \int \frac{d p_{2}^{0} d^{3} p_{2}}{i(2 \pi)^{4}} \frac{d p_{3}^{0} d^{3} p_{3}}{i(2 \pi)^{4}} \bar{u}\left(p_{s}\right) \bar{u}\left(p_{r}\right) \bar{u}\left(p_{f}\right) \frac{\Gamma_{N N}^{+}\left(p_{2}^{\prime}, p_{3}\right) \cdot u\left(p_{2}^{\prime}\right) \bar{u}\left(p_{2}^{\prime}\right)}{p_{2}^{\prime 2}-m^{2}+i \varepsilon} \times \\
& \times \frac{F_{N N}^{\mathrm{a}}\left(p_{2}^{\prime}-p_{2}\right) \cdot u\left(p_{1}+q\right) \bar{u}\left(p_{1}+q\right)}{\left(p_{1}+q\right)^{2}-m^{2}+i \varepsilon} \cdot \Gamma_{\gamma^{*} N}^{\mu} \cdot \frac{u\left(p_{3}\right) \bar{u}\left(p_{3}\right)}{p_{3}^{2}-m^{2}+i \varepsilon} \times \\
& \times \frac{u\left(p_{2}\right) \bar{u}\left(p_{2}\right)}{p_{2}^{2}-m^{2}+i \varepsilon} \cdot \frac{u\left(p_{1}\right) \bar{u}\left(p_{1}\right)}{p_{1}^{2}-m^{2}+i \varepsilon} \cdot \Gamma_{{ }^{2} \mathrm{HeNNN}}\left(p_{1}, p_{2}, p_{3}\right) \chi^{3 \mathrm{He}} .
\end{aligned}
$$

Similarly to eq.(29) one can perform the integration over the $d p_{2,3}^{0}$ by taking residues over the poles in the nucleon propagators $D^{-1}\left(p_{2}\right)$ and $D^{-1}\left(p_{3}\right)$. In contrast to IA case we now need to consider propogators $D^{-1}\left(p_{1}\right), D^{-1}\left(p_{1}+q\right)$ and $D^{-1}\left(p_{2}^{\prime}\right)$ which are also depending on variables $p_{2}^{0}, p_{3}^{0}$ as given by eq.(51). We observe, however, 
that poles of those propagators are all located in the upper part of the $p_{2,3}^{0}$ complex planes so we can close the contour in the lower half-planes containing single poles $+\sqrt{\left|\mathbf{p}_{2}\right|^{2}+m^{2}}$ and $+\sqrt{\left|\mathbf{p}_{3}\right|^{2}+m^{2}}$, respectively. In addition, when talking about $d p_{2}^{0}$ integration it is necessary to point out that $F_{N N}^{\mathrm{a}}\left(p_{2}^{\prime}-p_{2}\right)$ does not have singularity in $p_{2}^{0}$. It reflects the fact that at high energies the total cross section of $N N$ interaction depends only weakly on the collision energy.

Thus, the integration results in substitution

$$
\int_{-\infty}^{\infty} \frac{d p_{2,3}^{0}}{p_{2,3}^{2}-m^{2}+i \varepsilon}=-\frac{2 \pi i}{2 \sqrt{\left|\mathbf{p}_{2,3}\right|^{2}+m^{2}}} \approx-\frac{2 \pi i}{2 m}
$$

After this replacement and some arrangement of Dirac spinors eq.(53) is going to look like

$$
\begin{aligned}
A_{1 \mathrm{a}}^{\mu} & =-\frac{1}{(2 m)^{2}} \sum_{\text {spins }} \int \frac{d^{3} p_{2}}{(2 \pi)^{3}} \frac{d^{3} p_{3}}{(2 \pi)^{3}} \frac{\bar{u}\left(p_{r}\right) \bar{u}\left(p_{s}\right) \Gamma_{N N}^{+}\left(p_{2}^{\prime}, p_{3}\right) u\left(p_{2}^{\prime}\right) u\left(p_{3}\right)}{p_{2}^{\prime 2}-m^{2}+i \varepsilon} \times \\
& \times \frac{\bar{u}\left(p_{f}\right) \bar{u}\left(p_{2}^{\prime}\right) F_{N N}^{\mathrm{a}}\left(p_{2}^{\prime}-p_{2}\right) u\left(p_{1}+q\right) u\left(p_{2}\right)}{\left(p_{1}+q\right)^{2}-m^{2}+i \varepsilon} \cdot \bar{u}\left(p_{1}+q\right) \Gamma_{\gamma^{*} N}^{\mu} u\left(p_{1}\right) \times \\
& \times \frac{\bar{u}\left(p_{3}\right) \bar{u}\left(p_{2}\right) \bar{u}\left(p_{1}\right) \Gamma_{{ }^{3} \mathrm{HeNNN}}\left(p_{1}, p_{2}, p_{3}\right) \chi^{3} \mathrm{He}}{p_{1}^{2}-m^{2}+i \varepsilon} .
\end{aligned}
$$

After $p_{2}^{0}, p_{3}^{0}$ integrations were performed, the diagram of Fig. 11(a) becomes the noncovariant time ordered diagram (from left to right), where a virtual photon is absorbed by the target nucleon, and then the produced fast nucleon rescatters off a spectator nucleon. The definition of the momentum space wave functions is now straightforward. It corresponds to the nonrelativistic reduction of the nuclear vertices $\Gamma_{{ }^{3} \mathrm{He}}$ and $\Gamma_{N N}^{+}$as given by eqs. (34) and (36). The third term in eq.(55) can be replaced by electromagnetic current, just like we did in eq.(37) for impulse ap- 
proximation diagram. Finally we are also able to connect $F_{N N}^{\mathrm{a}}$ rescattering vertex function to the nonrelativistic amplitude of nucleon-nucleon scattering (see eq.(21)):

$$
\begin{aligned}
\bar{u}\left(p_{f}\right) \bar{u}\left(p_{2}^{\prime}\right) F_{N N}^{\mathrm{a}} u\left(p_{1}+q\right) u\left(p_{2}\right) & =\sqrt{s\left(s-4 m^{2}\right)} f_{d i f f}^{\mathrm{a}}\left(p_{2}^{\prime}-p_{2}\right) \delta_{\alpha, \alpha^{\prime}} \delta_{\beta, \beta^{\prime}} \\
& \approx s f_{d i f f}^{\mathrm{a}}\left(p_{2}^{\prime}-p_{2}\right) \delta_{\alpha, \alpha^{\prime}} \delta_{\beta, \beta^{\prime}}
\end{aligned}
$$

where $s$ is a total invariant energy. $\delta_{\alpha, \alpha^{\prime}}, \delta_{\beta, \beta^{\prime}}$ replace spins before the collision to the spin values after the collision in the sum $\sum_{\text {spins }}$. For small center-of-mass angle and high energy rescatterings, which are characteristic features for fast knocked outslow spectator nucleons interaction, $f_{d i f f}^{\mathrm{a}}$ diffraction amplitude can be constructed via parametrization of proton-nucleon and proton-proton experimental data. Corresponding parameters are total cross-section $\sigma_{\text {tot }}$, slope parameter $B$ and Re to Im amplitude ratio $\alpha$ :

$$
f_{\text {diff }}^{\mathrm{a}}\left(p_{2}^{\prime}-p_{2}\right)_{\perp}=\sigma_{\text {tot }}(i+\alpha) e^{-\frac{B}{2}\left(p_{2}^{\prime}-p_{2}\right)_{\perp}^{2}}
$$

where $\perp$ denotes that soft scattering amplitude mainly depends on $x$ and $y$ components of transferred momentum. Thus, non-relativistic reduction rewrites $A_{1 \mathrm{a}}^{\mu}$ as

$$
\begin{aligned}
A_{1 \mathrm{a}}^{\mu} & =-F \frac{1}{(2 m)} \int \frac{d^{3} p_{2}}{(2 \pi)^{3}} d^{3} p_{3} \Psi_{\mathbf{k}_{r s}}^{(+)}\left(\frac{p_{2}^{\prime}-p_{3}}{2}\right) \times \\
& \times \frac{s f_{d i f f}^{\mathrm{a}}\left(p_{2}^{\prime}-p_{2}\right)_{\perp}}{\left(p_{1}+q\right)^{2}-m^{2}+i \varepsilon} \cdot j^{\mu}\left(p_{1}+q, p_{1}\right) \cdot \Psi_{{ }^{3} \mathrm{He}}\left(p_{1} ; p_{2} ; p_{3}\right) .
\end{aligned}
$$

with $F=2 E_{r} 2 E_{s}$ being normalization constant. Let us analyze the propagator $-D\left(p_{1}+q\right) \equiv\left(p_{1}+q\right)^{2}-m^{2}+i \varepsilon$ as we express $p_{1}$ through the relation of eq.(51)

$$
\begin{aligned}
-D\left(p_{1}+q\right) & =M_{3_{\mathrm{He}}}^{2}-2 P_{{ }^{3} \mathrm{He}}\left(p_{2}+p_{3}\right)+\left(p_{2}+p_{3}\right)^{2}+ \\
& +2 q\left(P_{{ }^{3} \mathrm{He}}-p_{2}-p_{3}\right)-Q^{2}-m^{2}+i \varepsilon, \quad Q^{2} \equiv-q^{2} .
\end{aligned}
$$


Using the 4-vector conservation condition for quasielastic scattering

$$
\left(M_{3_{\mathrm{He}}}, 0\right)+\left(q_{0}, \mathbf{q}\right)=\left(E_{f}, \mathbf{p}_{f}\right)+\left(E_{r}, \mathbf{p}_{r}\right)+\left(E_{s}, \mathbf{p}_{s}\right)
$$

we can transform eq.(59) replacing $m^{2}$ by the following expression:

$$
\begin{aligned}
m^{2} & =\left(q+P_{3_{\mathrm{H}}}-p_{r}-p_{s}\right)^{2}= \\
& =M_{3_{\mathrm{He}}}^{2}-2 P_{3_{\mathrm{He}}}\left(p_{r}+p_{s}\right)+2 m^{2}+2 q\left(P_{3_{\mathrm{He}}}-p_{r}-p_{s}\right)-Q^{2} .
\end{aligned}
$$

This yields

$$
\begin{aligned}
-D\left(p_{1}+q\right) & =\left(p_{2}+p_{3}\right)^{2}-2 M^{3_{\mathrm{He}}}\left(E_{2}+E_{3}\right)-2 q_{0}\left(E_{2}+E_{3}\right)+2 \mathbf{q}\left(\mathbf{p}_{2}+\mathbf{p}_{3}\right)- \\
& -2 m^{2}+2 M^{3 \mathrm{He}}\left(E_{r}+E_{s}\right)+2 q_{0}\left(E_{r}+E_{s}\right)-2 \mathbf{q}\left(\mathbf{p}_{r}+\mathbf{p}_{s}\right)+i \varepsilon
\end{aligned}
$$

or, chosing as usually transferred momentum $\mathbf{q}$ in the $z$ direction, $\mathbf{q}=\left(0,0, q_{\mathbf{v}}\right)$, we derive the following form for knocked out nucleon propagator:

$$
\begin{aligned}
-D\left(p_{1}+q\right) & =2 q_{\mathrm{v}}\left[\left(p_{2 z}+p_{3 z}\right)-\left(p_{s z}+p_{r z}\right)+\frac{q_{0}}{q_{\mathrm{v}}}\left(E_{s}+E_{r}-E_{2}-E_{3}\right)+\right. \\
& \left.+\frac{M_{3} \mathrm{He}}{q_{\mathrm{v}}}\left(E_{s}+E_{r}-E_{2}-E_{3}\right)+\frac{\left(p_{2}+p_{3}\right)^{2}-2 m^{2}}{2 q_{\mathrm{v}}}+i \varepsilon\right] .
\end{aligned}
$$

For fixed $x_{B j} \equiv Q^{2} / 2 q_{0} m$ Bjorken parameter and increasing values of transferred momentum $q_{\mathrm{v}}$ only first three terms are important in this expression. Contribution of last two terms at fixed recoil energy is of the order of $\mathcal{O}\left(1 / q_{\mathrm{v}}\right)$ and they are vanishing as we consider higher and higher $q_{\mathrm{v}}$. Therefore

$$
-D\left(p_{1}+q\right) \approx 2 q_{\mathrm{v}}\left[\left(p_{2 z}+p_{3 z}\right)-\left(p_{s z}+p_{r z}\right)+\Delta_{0}+i \varepsilon\right],
$$

where

$$
\Delta_{0}=\frac{q_{0}}{q_{\mathrm{v}}}\left(E_{s}+E_{r}-E_{2}-E_{3}\right) .
$$


We now observe from eq.(60) that $-\left(\mathbf{p}_{r}+\mathbf{p}_{s}\right)=\mathbf{p}_{f}-\mathbf{q} \equiv \mathbf{p}_{m}$ with $\mathbf{p}_{m}$ being a missing momentum. Recalling also $\mathbf{p}_{1}=-\left(\mathbf{p}_{2}+\mathbf{p}_{3}\right)$ we rewrite eq.(64) as

$$
-D\left(p_{1}+q\right)=2 q_{\mathrm{v}}\left[p_{m z}-p_{1 z}+\Delta_{0}+i \varepsilon\right]
$$

where now

$$
\Delta_{0}=\frac{q_{0}}{q_{\mathrm{v}}}\left(E_{1}+E_{s}+E_{r}-M_{3 \mathrm{He}}\right) \approx \frac{q_{0}}{q_{\mathrm{v}}}\left(m+E_{s}+E_{r}-M_{3 \mathrm{He}}\right) \equiv \frac{q_{0}}{q_{\mathrm{v}}} E_{m} .
$$

Here we have neglected term $\left|\mathbf{p}_{1}\right|^{2} / 2 m^{2} \ll 1$ in expansion for $E_{1}$, thus replacing it with $m$ in the nonrelativistic limit. It is important to mention, that in the lab-frame description without such a neglection one cannot justify the closure over intermediate nuclear states we performed earlier in eq.(53) for the spinor $\left[\hat{p}_{1}+\hat{q}+m\right]$.

The missing energy $E_{m}$, which is also given by $E_{m}=q_{0}+m-\sqrt{m^{2}+p_{f}^{2}}$ in case of the ${ }^{3}$ He three-body break-up kinematics has very clear physical meaning:

$$
E_{m}=T_{N N}+\left|\epsilon_{b}\right|
$$

with $T_{N N}$ as a kinetic energy of the spectator two-nucleon system and $\left|\epsilon_{b}\right|$ as a modulus of the target binding energy.

Observing that in high energy limit $2 m q_{\mathrm{v}} \approx s$ we obtain from eq.(58) the following expression for single rescattering amplitude

$$
\begin{aligned}
A_{1 \mathrm{a}}^{\mu} & =-\frac{F}{2} \int \frac{d^{3} p_{2}}{(2 \pi)^{3}} d^{3} p_{3} \Psi_{\mathbf{k}_{r s}}^{(+)}\left(\frac{p_{2}^{\prime}-p_{3}}{2}\right) \times \\
& \times \frac{f_{d i f f}^{\mathrm{a}}\left(p_{2}^{\prime}-p_{2}\right)_{\perp}}{p_{m z}-p_{1 z}+\Delta_{0}+i \varepsilon} \cdot j^{\mu}\left(p_{1}+q, p_{1}\right) \cdot \Psi_{3_{\mathrm{He}}}\left(p_{1} ; p_{2} ; p_{3}\right) .
\end{aligned}
$$


Defining new variable $\mathbf{k}=\mathbf{p}_{1}-\mathbf{p}_{m}$, where $\mathbf{p}_{m} \equiv \mathbf{p}_{f}-\mathbf{q}=-\mathbf{p}_{r}-\mathbf{p}_{s}$, we change variables in eq.(69)

$$
\begin{array}{ll}
\mathbf{p}_{1}=\mathbf{k}+\mathbf{p}_{m} & \text { from definition, } \\
\mathbf{p}_{\mathbf{2}}=-\mathbf{k}-\mathbf{p}_{m}-\mathbf{p}_{3} & \text { from eq.(51), } \\
\mathbf{p}_{\mathbf{2}}^{\prime}=\mathbf{p}_{r}+\mathbf{p}_{s}-\mathbf{p}_{3}=-\mathbf{p}_{m}-\mathbf{p}_{3} & \text { from } p_{2}^{\prime}+p_{3}=p_{r}+p_{s}
\end{array}
$$

After replacement we get:

$$
\begin{aligned}
A_{1 \mathrm{a}}^{\mu} & =-\frac{F}{2} \int \frac{d^{3} k}{(2 \pi)^{3}} d^{3} p_{3} \Psi_{\mathbf{k}_{r s}}^{(+)}\left(\frac{-p_{m}-2 p_{3}}{2}\right) \frac{f_{d i f f}^{\mathrm{a}}\left(k_{\perp}\right)}{-k_{z}+\Delta_{0}+i \varepsilon} \times \\
& \times j^{\mu}\left(k+p_{m}+q, k+p_{m}\right) \cdot \Psi_{3_{\mathrm{He}}}\left(k+p_{m} ;-k-p_{3}-p_{m} ; p_{3}\right) .
\end{aligned}
$$

It is convenient to change $p_{3}$ variable as well, replacing it with the relative momentum of spectator pair before their interaction in $\Gamma_{N N}^{+}$vertex. The corresponding relations are $\mathbf{k}_{2^{\prime} 3}=\left(\mathbf{p}_{2}^{\prime}-\mathbf{p}_{3}\right) / 2=-\mathbf{p}_{m} / 2-\mathbf{p}_{3}$. The result is

$$
\begin{aligned}
A_{1 \mathrm{a}}^{\mu} & =-\frac{F}{2} \int \frac{d^{3} k}{(2 \pi)^{3}} d^{3} k_{2^{\prime} 3} \Psi_{\mathbf{k}_{r s}}^{(+)}\left(k_{2^{\prime} 3}\right) \frac{f_{\text {diff }}^{\mathrm{a}}\left(k_{\perp}\right)}{-k_{z}+\Delta_{0}+i \varepsilon} \times \\
& \times j^{\mu}\left(k+p_{m}+q, k+p_{m}\right) \times \\
& \times \quad \Psi_{{ }^{3} \mathrm{He}}\left(k+p_{m} ;-k+k_{2^{\prime} 3}-p_{m} / 2 ;-k_{2^{\prime} 3}-p_{m} / 2\right) .
\end{aligned}
$$

We are now ready to perform $k_{z}$ integration. From eq.(44)

$$
\frac{1}{-k_{z}+\Delta_{0}+i \varepsilon}=-i \pi \delta\left(k_{z}-\Delta_{0}\right)+\mathrm{P} \cdot \mathrm{V} \cdot \frac{1}{-k_{z}+\Delta_{0}}
$$

which represents $A_{1 \mathrm{a}}^{\mu}$ as a sum of two terms:

$$
A_{1 \mathrm{a}}^{\mu(\text { pole })}=i \frac{F}{2} \int \frac{d^{2} k_{\perp}}{2(2 \pi)^{2}} d^{3} k_{2^{\prime} 3} \Psi_{\mathbf{k}_{r s}}^{(+)}\left(k_{2^{\prime} 3}\right) f_{d i f f}^{\mathrm{a}}\left(k_{\perp}\right) \times
$$




$$
\begin{aligned}
& \times j^{\mu}\left(k_{\Delta_{0}}+p_{m}+q, k_{\Delta_{0}}+p_{m}\right) \times \\
& \times \quad \Psi_{{ }^{\mathrm{He}}}\left(k_{\Delta_{0}}+p_{m} ;-k_{\Delta_{0}}+k_{2^{\prime} 3}-p_{m} / 2 ;-k_{2^{\prime} 3}-p_{m} / 2\right),
\end{aligned}
$$

where $\mathbf{k}_{\Delta_{0}}=\left(k_{\perp}, \Delta_{0}\right)$ and

$$
\begin{aligned}
A_{1 \mathrm{a}}^{\mu(\text { P.V. })} & =-\frac{F}{2} \mathrm{P} . \mathrm{V} \cdot \int \frac{d k_{z}}{2 \pi} \int \frac{d^{2} k_{\perp}}{(2 \pi)^{2}} d^{3} k_{2^{\prime} 3} \Psi_{\mathbf{k}_{r s}}^{(+)}\left(k_{2^{\prime} 3}\right) \frac{f_{d i f f}^{\mathrm{a}}\left(k_{\perp}\right)}{-k_{z}+\Delta_{0}} \times \\
& \times j^{\mu}\left(k+p_{m}+q, k+p_{m}\right) \times \\
& \times \Psi_{{ }^{\mathrm{He}}}\left(k+p_{m} ;-k+k_{2^{\prime} 3}-p_{m} / 2 ;-k_{2^{\prime} 3}-p_{m} / 2\right) .
\end{aligned}
$$

In its turn each of these terms splits into two terms as we use eq.(41) for continuum wave function $\Psi_{\mathbf{k}_{r s}}^{(+)}\left(k_{2^{\prime} 3}\right)$ :

$$
\begin{aligned}
& A_{1 \mathrm{a}}^{\mu(\text { pole }, 0)}=i \pi \frac{F}{2} \int d^{2} k_{\perp} f_{\text {diff }}^{\mathrm{a}}\left(k_{\perp}\right) \cdot j^{\mu}\left(k_{\Delta_{0}}+p_{m}+q, k_{\Delta_{0}}+p_{m}\right) \times \\
& \times \Psi_{3 \mathrm{He}}\left(k_{\Delta_{0}}+p_{m} ;-k_{\Delta_{0}}+k_{r s}-p_{m} / 2 ;-k_{r s}-p_{m} / 2\right), \\
& A_{1 \mathrm{a}}^{\mu(\mathrm{P} . \mathrm{V} ., 0)}=-\frac{F}{2} \mathrm{P} . \mathrm{V} \cdot \int d k_{z} \int d^{2} k_{\perp} \frac{f_{d i f f}^{\mathrm{a}}\left(k_{\perp}\right)}{-k_{z}+\Delta_{0}} \times \\
& \times j^{\mu}\left(k+p_{m}+q, k+p_{m}\right) \times \\
& \times \quad \Psi_{{ }^{3} \mathrm{He}}\left(k+p_{m} ;-k+k_{r s}-p_{m} / 2 ;-k_{r s}-p_{m} / 2\right), \\
& A_{1 \mathrm{a}}^{\mu(\text { pole }, s)}=i \frac{F}{2} \int \frac{d^{2} k_{\perp}}{(2 \pi)^{2}} d^{3} k_{2^{\prime} 3} \frac{f_{N N}^{\text {off shell, a }}\left(k_{r s}, k_{2^{\prime} 3}\right)}{k_{2^{\prime} 3}^{2}-k_{r s}^{2}-i \varepsilon} \times \\
& \times f_{\text {diff }}^{\mathrm{a}}\left(k_{\perp}\right) j^{\mu}\left(k_{\Delta_{0}}+p_{m}+q, k_{\Delta_{0}}+p_{m}\right) \times \\
& \times \Psi_{{ }^{3} \mathrm{He}}\left(k_{\Delta_{0}}+p_{m} ;-k_{\Delta_{0}}+k_{2^{\prime} 3}-p_{m} / 2 ;-k_{2^{\prime} 3}-p_{m} / 2\right),
\end{aligned}
$$

and

$$
A_{1 \mathrm{a}}^{\mu(\mathrm{P} . \mathrm{V} ., s)}=-\frac{F}{2} \mathrm{P} . \mathrm{V} \cdot \int \frac{d k_{z}}{2 \pi} \int \frac{d^{2} k_{\perp}}{\pi} d^{3} k_{2^{\prime} 3} \frac{f_{N N}^{\mathrm{off} \text { shell, a }}\left(k_{r s}, k_{2^{\prime} 3}\right)}{k_{2^{\prime} 3}^{2}-k_{r s}^{2}-i \varepsilon} \times
$$




$$
\begin{aligned}
& \times \frac{f_{\text {diff }}^{\mathrm{a}}\left(k_{\perp}\right)}{-k_{z}+\Delta_{0}} \cdot j^{\mu}\left(k+p_{m}+q, k+p_{m}\right) \times \\
& \times \quad \Psi_{3_{\mathrm{He}}}\left(k+p_{m} ;-k+k_{2^{\prime} 3}-p_{m} / 2 ;-k_{2^{\prime} 3}-p_{m} / 2\right) .
\end{aligned}
$$

For the last two terms we can present $d^{3} k_{2^{\prime} 3}=1 / 2\left|\mathbf{k}_{2^{\prime} 3}\right| d k_{2^{\prime} 3}^{2} d \Omega_{2^{\prime} 3}$ and proceed simmilar to what we did for IA pair distortion amplitude $A_{0 s}^{\mu}$ (see eqs.(45)-(48)). As before, keeping pole term only and defining $\mathbf{k}_{r s}^{\prime}=\left|\mathbf{k}_{r s}\right|\left(\mathbf{k}_{2^{\prime} 3} /\left|\mathbf{k}_{2^{\prime} 3}\right|\right)$ we get

$$
\begin{aligned}
& A_{1 \mathrm{a}}^{\mu(\text { pole }, s-\text { pole })}=i \frac{F}{2} \int \frac{d^{2} k_{\perp}}{(2 \pi)^{2}} \frac{d \Omega_{2^{\prime} 3}}{4} \tilde{f}_{N N}^{\text {on shell, a }}\left(k_{r s}, k_{r s}^{\prime}\right) \times \\
& \times f_{\text {diff }}^{\mathrm{a}}\left(k_{\perp}\right) j^{\mu}\left(k_{\Delta_{0}}+p_{m}+q, k_{\Delta_{0}}+p_{m}\right) \times \\
& \times \quad \Psi_{3 \mathrm{He}}\left(k_{\Delta_{0}}+p_{m} ;-k_{\Delta_{0}}+k_{2^{\prime} 3}^{\prime}-p_{m} / 2 ;-k_{2^{\prime} 3}^{\prime}-p_{m} / 2\right), \\
& A_{1 \mathrm{a}}^{\mu(\mathrm{P} . \mathrm{V} ., s \text {-pole })}=-\frac{F}{2} \mathrm{P} . \mathrm{V} \cdot \int \frac{d k_{z}}{2 \pi} \int \frac{d^{2} k_{\perp}}{\pi} \frac{d \Omega_{2^{\prime} 3}}{4} \tilde{f}_{N N}^{\text {on shell, a }}\left(k_{r s}, k_{r s}^{\prime}\right) \times \\
& \times \frac{f_{\text {diff }}^{\mathrm{a}}\left(k_{\perp}\right)}{-k_{z}+\Delta_{0}} \cdot j^{\mu}\left(k+p_{m}+q, k+p_{m}\right) \times \\
& \times \quad \Psi_{3_{\mathrm{He}}}\left(k+p_{m} ;-k+k_{2^{\prime} 3}^{\prime}-p_{m} / 2 ;-k_{2^{\prime} 3}^{\prime}-p_{m} / 2\right) .
\end{aligned}
$$

To complete the calculation of single rescattering amplitude one should calculate also the amplitude $A_{1 \mathrm{~b}}^{\mu}$ corresponding to the diagram of Figure 11(b), where the leading nucleon rescatters second spectator nucleon. To do this we need to interchange the momenta of nucleons " 2 " and " 3 " in the eqs. (76), (77), (82), and (83). For the sake of brevity, however, we will do it in initial (unsimplified) expression for full $A_{1 \mathrm{a}}^{\mu}$, given by eq.(74), where we interchange second and third arguments of the ${ }^{3} \mathrm{He}$ wave function as well as replace everywhere $p_{2}^{\prime} \rightarrow p_{3}^{\prime}$ and $p_{2} \leftrightarrow p_{3}$. This will introduce variable $k_{3^{\prime} 2}$ which is handy to replace by $k_{3^{\prime} 2}=-k_{2^{\prime} 3}$. We then can merge both integrals for $A_{1 \mathrm{a}}^{\mu}$ 
and $A_{1 \mathrm{~b}}^{\mu}$ chosing notation $d^{3} k_{23}^{\prime}$ rather than $d^{3} k_{2^{\prime} 3}$ or $d^{3} k_{23^{\prime}}$ for integration variable corresponding to the distortion of spectators. Our final result is:

$$
\begin{aligned}
A_{1}^{\mu} & =A_{1 \mathrm{a}}^{\mu}+A_{1 \mathrm{~b}}^{\mu}=-\frac{F}{2} \int \frac{d^{3} k}{(2 \pi)^{3}} d^{3} k_{23}^{\prime} \Psi_{\mathbf{k}_{r s}}^{(+)}\left(k_{23}^{\prime}\right) j^{\mu}\left(k+p_{m}+q, k+p_{m}\right) \times \\
& \times\left[\frac{f_{\text {diff }}^{\mathrm{a}}\left(k_{\perp}\right)}{-k_{z}+\Delta_{0}+i \varepsilon} \Psi_{3_{\mathrm{He}}}\left(k+p_{m} ;-k+k_{23}^{\prime}-p_{m} / 2 ;-k_{23}^{\prime}-p_{m} / 2\right)+\right. \\
& \left.+\frac{f_{\text {diff }}^{\mathrm{b}}\left(k_{\perp}\right)}{-k_{z}+\Delta_{0}+i \varepsilon} \Psi_{3_{\mathrm{He}}}\left(k+p_{m} ; k_{23}^{\prime}-p_{m} / 2 ;-k-k_{23}^{\prime}-p_{m} / 2\right)\right] .
\end{aligned}
$$




\subsection{Double Rescattering Amplitudes}

This section is concerned with the double rescattering amplitude. The diagrams of Fig.12(a) and 12(b) describe the process where the fast $\left(p_{f}\right)$ knocked out nucleon rescatters sequentially off both spectator nucleons. The general expression with suppressed spinors for the amplitude corresponding to the diagram in Fig. 12(a) is given by $n=2$ term of eq.(23) as follows:

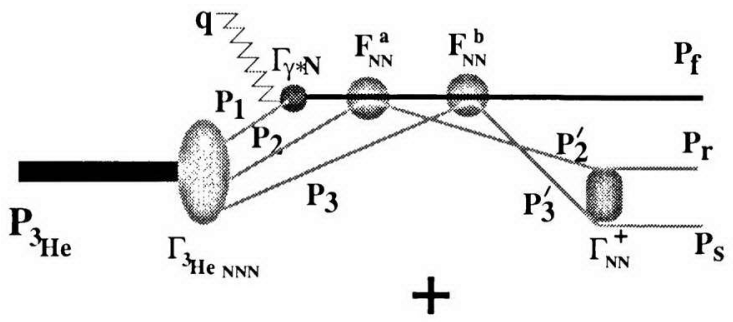

(a)

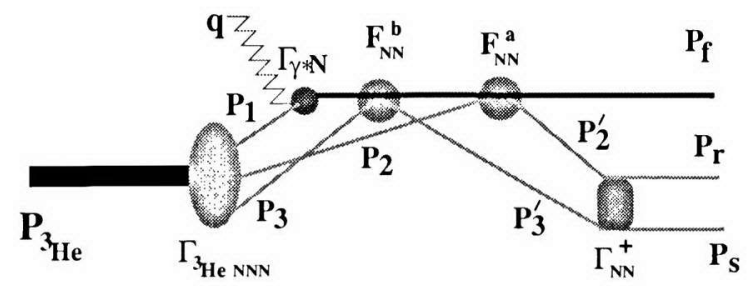

Figure 12: Double Rescattering Diagrams.

$$
\begin{aligned}
A_{2 \mathrm{a}}^{\mu} & =\int d^{4} p_{1} d^{4} p_{2} d^{4} p_{3} d^{4} p_{2}^{\prime} d^{4} p_{3}^{\prime} \frac{1}{\left[i(2 \pi)^{4}\right]^{3}} \delta^{4}\left(p_{1}+p_{2}+p_{3}-P_{{ }^{H} \mathrm{He}}\right) \times \\
& \times \delta^{4}\left(p_{2}^{\prime}+p_{3}^{\prime}-p_{r}-p_{s}\right) \cdot \frac{\Gamma_{N N}^{+}\left(p_{2}^{\prime}, p_{3}^{\prime}\right)}{D\left(p_{2}^{\prime}\right) D\left(p_{3}^{\prime}\right)} \cdot \frac{F_{N N}^{\mathrm{b}}\left(p_{3}^{\prime}-p_{3}\right)}{D\left(p_{1}+q+p_{2}+p_{2}^{\prime}\right)} \times \\
& \times \frac{F_{N N}^{\mathrm{a}}\left(p_{2}^{\prime}-p_{2}\right)}{D\left(p_{1}+q\right)} \cdot \Gamma_{\gamma^{*} N}^{\mu} \cdot \frac{\Gamma_{3 \mathrm{He} N N}\left(p_{1}, p_{2}, p_{3}\right)}{D\left(p_{1}\right) D\left(p_{2}\right) D\left(p_{3}\right)},
\end{aligned}
$$

Contrary to the single rescattering case, before their interaction in $\Gamma_{N N}^{+}$vertex both spectators underwent collision with the leading nucleon and therefore they are indis- 
tinguishable. The factor $1 /[n !(A-n-1) !]=1 / 2$ already canceled here to prevent double counting while considering contribution of Fig.12(a) diagram. 4-dimensional delta-function integrations get rid of $d^{4} p_{1}, d^{4} p_{2}^{\prime}$ integrals and also result to energymomentum conservation relations

$$
p_{1}=P_{3_{\mathrm{He}}}-p_{2}-p_{3}, \quad p_{2}^{\prime}=p_{r}+p_{s}-p_{3}^{\prime} .
$$

"Minus" sign convention used to define propagators $D$ helps us to calculate the sign of the amplitude - there are seven $D(p)$ s, so sign is going to be $(-1)^{7}=-1$. Rewriting eq.(85) with explicit spinor dependence we have

$$
\begin{aligned}
A_{2 \mathrm{a}}^{\mu} & =\int \frac{-d^{4} p_{3}^{\prime}}{i(2 \pi)^{4}} \frac{d^{4} p_{2}}{i(2 \pi)^{4}} \frac{d^{4} p_{3}}{i(2 \pi)^{4}} \bar{u}\left(p_{s}\right) \bar{u}\left(p_{r}\right) \bar{u}\left(p_{f}\right) \frac{\Gamma_{N N}^{+}\left(p_{2}^{\prime}, p_{3}^{\prime}\right)\left[\hat{p}_{2}^{\prime}+m\right]\left[\hat{p}_{3}^{\prime}+m\right]}{\left(p_{2}^{\prime 2}-m^{2}+i \varepsilon\right)\left(p_{3}^{\prime 2}-m^{2}+i \varepsilon\right)} \times \\
& \times \frac{F_{N N}^{\mathrm{b}}\left(p_{3}^{\prime}-p_{3}\right)\left[\hat{p}_{1}+\hat{q}+p_{2}-p_{2}^{\prime}+m\right]}{\left(p_{1}+q+p_{2}-p_{2}^{\prime}\right)^{2}-m^{2}+i \varepsilon} \times \\
& \times \frac{F_{N N}^{\mathrm{a}}\left(p_{2}^{\prime}-p_{2}\right)\left[\hat{p}_{1}+\hat{q}+m\right]}{\left(p_{1}+q\right)^{2}-m^{2}+i \varepsilon} \cdot \Gamma_{\gamma^{*} N}^{\mu} \cdot \frac{\left[\hat{p}_{3}+m\right]}{p_{3}^{2}-m^{2}+i \varepsilon} \times \\
& \times \frac{\left[\hat{p}_{2}+m\right]}{p_{2}^{2}-m^{2}+i \varepsilon} \cdot \frac{\left[\hat{p}_{1}+m\right]}{p_{1}^{2}-m^{2}+i \varepsilon} \cdot \Gamma_{{ }^{\mathrm{HeNNN}}}\left(p_{1}, p_{2}, p_{3}\right) \chi^{{ }^{3} \mathrm{He}} .
\end{aligned}
$$

Next step is to neglect off-shell effects in the nucleons' spinors and get advantage of the closure expansion ( eq.(27) ) which yields to the following:

$$
\begin{aligned}
A_{2 \mathrm{a}}^{\mu} & =-\sum_{\text {spins }} \int \frac{d^{4} p_{3}^{\prime}}{i(2 \pi)^{4}} \frac{d p_{2}^{0} d^{3} p_{2}}{i(2 \pi)^{4}} \frac{d p_{3}^{0} d^{3} p_{3}}{i(2 \pi)^{4}} \bar{u}\left(p_{s}\right) \bar{u}\left(p_{r}\right) \bar{u}\left(p_{f}\right) \times \\
& \times \frac{\Gamma_{N N}^{+}\left(p_{2}^{\prime}, p_{3}^{\prime}\right) \cdot u\left(p_{2}^{\prime}\right) \bar{u}\left(p_{2}^{\prime}\right) u\left(p_{3}^{\prime}\right) \bar{u}\left(p_{3}^{\prime}\right)}{\left(p_{2}^{\prime 2}-m^{2}+i \varepsilon\right)\left(p_{3}^{\prime 2}-m^{2}+i \varepsilon\right)} \times \\
& \times \frac{F_{N N}^{\mathrm{b}}\left(p_{3}^{\prime}-p_{3}\right) \cdot u\left(p_{1}+q+p_{2}-p_{2}^{\prime}\right) \bar{u}\left(p_{1}+p_{2}-p_{2}^{\prime}+q\right)}{\left(p_{1}+q+p_{2}-p_{2}^{\prime}\right)^{2}-m^{2}+i \varepsilon} \times \\
& \times \frac{F_{N N}^{\mathrm{a}}\left(p_{2}^{\prime}-p_{2}\right) \cdot u\left(p_{1}+q\right) \bar{u}\left(p_{1}+q\right)}{\left(p_{1}+q\right)^{2}-m^{2}+i \varepsilon} \cdot \Gamma_{\gamma^{*} N}^{\mu} \cdot \frac{u\left(p_{3}\right) \bar{u}\left(p_{3}\right)}{p_{3}^{2}-m^{2}+i \varepsilon} \times \\
& \times \frac{u\left(p_{2}\right) \bar{u}\left(p_{2}\right)}{p_{2}^{2}-m^{2}+i \varepsilon} \cdot \frac{u\left(p_{1}\right) \bar{u}\left(p_{1}\right)}{p_{1}^{2}-m^{2}+i \varepsilon} \cdot \Gamma^{{ }^{2} \mathrm{HeNNN}}\left(p_{1}, p_{2}, p_{3}\right) \chi^{3} \mathrm{He}
\end{aligned}
$$


where, as before, we have sum over all intermidiate spin-states.

Fixing nucleons with 4 -momenta $p_{2}, p_{3}$ and $p_{3}^{\prime}$ to be on their mass-shell we perform time-component integrations which are equivalent to the substitutions

$$
\int_{-\infty}^{\infty} \frac{d p_{3^{\prime}, 2,3}^{0}}{p_{3^{\prime}, 2,3}^{2}-m^{2}+i \varepsilon}=-\frac{2 \pi i}{2 \sqrt{\left|\mathbf{p}_{3^{\prime}, 2,3}\right|^{2}+m^{2}}} \approx-\frac{2 \pi i}{2 m}
$$

Appropriately arranging Dirac spinors in eq.(88) we present the resulting equation as

$$
\begin{aligned}
A_{2 \mathrm{a}}^{\mu} & =\frac{1}{(2 m)^{3}} \sum_{\text {spins }} \int \frac{d^{3} p_{3}^{\prime}}{(2 \pi)^{3}} \frac{d^{3} p_{2}}{(2 \pi)^{3}} \frac{d^{3} p_{3}}{(2 \pi)^{3}} \frac{\bar{u}\left(p_{r}\right) \bar{u}\left(p_{s}\right) \Gamma_{N N}^{+}\left(p_{2}^{\prime}, p_{3}^{\prime}\right) u\left(p_{2}^{\prime}\right) u\left(p_{3}^{\prime}\right)}{p_{2}^{\prime 2}-m^{2}+i \varepsilon} \times \\
& \times \frac{\bar{u}\left(p_{f}\right) \bar{u}\left(p_{3}^{\prime}\right) F_{N N}^{\mathrm{b}}\left(p_{3}^{\prime}-p_{3}\right) u\left(p_{1}+q+p_{2}-p_{2}^{\prime}\right) u\left(p_{3}\right)}{\left(p_{1}+q+p_{2}-p_{2}^{\prime}\right)^{2}-m^{2}+i \varepsilon} \times \\
& \times \frac{\bar{u}\left(p_{1}+q+p_{2}-p_{2}^{\prime}\right) \bar{u}\left(p_{2}^{\prime}\right) F_{N N}^{\mathrm{a}}\left(p_{2}^{\prime}-p_{2}\right) u\left(p_{1}+q\right) u\left(p_{2}\right)}{\left(p_{1}+q\right)^{2}-m^{2}+i \varepsilon} \times \\
& \times \bar{u}\left(p_{1}+q\right) \Gamma_{\gamma^{*} N}^{\mu} u\left(p_{1}\right) \cdot \frac{\bar{u}\left(p_{3}\right) \bar{u}\left(p_{2}\right) \bar{u}\left(p_{1}\right) \Gamma_{{ }^{H} \mathrm{HeNNN}}\left(p_{1}, p_{2}, p_{3}\right) \chi^{3} \mathrm{He}}{p_{1}^{2}-m^{2}+i \varepsilon}
\end{aligned}
$$

After "0" component integrations are performed diagram of Fig. 12(a) becomes a non-covariant time ordered diagram (from left to right), where a virtual photon is absorbed by the target nucleon, produced fast nucleon propagates further, scatters off $p_{2}$ spectator and then $p_{3}$ one. Reduction theorem prevents it to interact with, let say, $p_{2}^{\prime}$ particle - the corresponding amplitude is exactly zero. Thus, Double Rescattering amplitude accounts for the most general type of the final state interactions of the knocked out nucleon.

Similar to the previous sections we implement nonrelativistic reduction of the $\Gamma$ verteces and introduce electromagnetic current $j^{\mu}$ using eqs.(34), (36) and (37). We 
obtain

$$
\begin{aligned}
A_{2 \mathrm{a}}^{\mu} & =\frac{F}{(2 m)^{2}} \int \frac{d^{3} p_{3}^{\prime}}{(2 \pi)^{3}} \frac{d^{3} p_{2}}{(2 \pi)^{3}} d^{3} p_{3} \Psi_{\mathbf{k}_{r s}}^{(+)}\left(\frac{p_{2}^{\prime}-p_{3}^{\prime}}{2}\right) \times \\
& \times \frac{s_{b} f_{\text {diff }}^{\mathrm{b}}\left(p_{3}^{\prime}-p_{3}\right)_{\perp}}{\left(p_{1}+q+p_{2}-p_{2}^{\prime}\right)^{2}-m^{2}+i \varepsilon} \times \\
& \times \frac{s_{a} f_{\text {diff }}^{\mathrm{a}}\left(p_{2}^{\prime}-p_{2}\right)_{\perp}}{\left(p_{1}+q\right)^{2}-m^{2}+i \varepsilon} \cdot j^{\mu}\left(p_{1}+q, p_{1}\right) \cdot \Psi_{3_{\mathrm{He}}}\left(p_{1} ; p_{2} ; p_{3}\right),
\end{aligned}
$$

where $s_{b}$ and $s_{a}$ are total invariant energies for nucleons coupling in verteces $F_{N N}^{\mathrm{b}}$ and $F_{N N}^{\mathrm{a}}$.

We now turn our attention to the propagators in eq.(91). We already dealt with the last one of them in the previous section. It's given by eq.(66)

$$
-D\left(p_{1}+q\right)=2 q_{\mathrm{v}}\left[p_{m z}-p_{1 z}+\Delta_{0}+i \varepsilon\right]
$$

For the former one we can use both conservation relations of eq.(86) and obtain

$$
-D\left(p_{1}+q+p_{2}-p_{2}^{\prime}\right)=\left(P_{3_{\mathrm{He}}}-p_{3}+q-p_{r}-p_{s}+p_{3}^{\prime}\right)^{2}-m^{2}+i \varepsilon
$$

Observing that due to the kinematic condition for quasielastic scattering we have $\left(q+P_{{ }^{3} \mathrm{He}}-p_{r}-p_{s}\right)^{2}=p_{f}^{2}=m^{2}$ eq.(93) transforms to

$$
\begin{aligned}
-D\left(p_{1}+q+p_{2}-p_{2}^{\prime}\right) & =2\left(p_{3}^{\prime}-p_{3}\right)\left(P_{3 \mathrm{He}}+q-p_{r}-p_{s}\right)+\left(p_{3}^{\prime}-p_{3}\right)^{2}+i \varepsilon= \\
& =2 q_{\mathrm{v}}\left[\frac{q_{0}}{q_{\mathrm{v}}}\left(E_{3}^{\prime}-E_{3}\right)-\left(p_{3 z}^{\prime}-p_{3 z}\right)+i \varepsilon+\right. \\
& \left.+\frac{1}{q_{\mathrm{v}}}\left(2\left(p_{3}^{\prime}-p_{3}\right)\left(P_{3 \mathrm{He}}-p_{r}-p_{s}\right)+\left(p_{3}^{\prime}-p_{3}\right)^{2}\right)\right]
\end{aligned}
$$

or, keeping only the terms which are not vanishing for large $q_{\mathrm{v}}$ while $x_{B j}$ kept fixed we arrive at

$$
-D\left(p_{1}+q+p_{2}-p_{2}^{\prime}\right) \approx 2 q_{\mathrm{v}}\left[\Delta_{3}-\left(p_{3 z}^{\prime}-p_{3 z}\right)+i \varepsilon\right]
$$


where

$$
\Delta_{3}=\frac{q_{0}}{q_{\mathrm{v}}}\left(E_{3}^{\prime}-E_{3}\right) .
$$

Based on the kinematic conditions of eq.(2) one can approximate $s_{a} \approx s_{b} \approx$ $2 m p_{f z} \approx 2 m q$. Canceling $s_{a} /\left(2 q_{\mathrm{v}} m\right) \approx 1$ and $s_{b} /\left(2 q_{\mathrm{v}} m\right) \approx 1$ one gets

$$
\begin{aligned}
A_{2 \mathrm{a}}^{\mu} & =\frac{F}{4} \int \frac{d^{3} p_{3}^{\prime}}{(2 \pi)^{3}} \frac{d^{3} p_{2}}{(2 \pi)^{3}} \frac{d^{3} p_{3}}{(2 \pi)^{3}} \Psi_{\mathbf{k}_{r s}}^{(+)}\left(\frac{p_{2}^{\prime}-p_{3}^{\prime}}{2}\right) \cdot \frac{f_{d i f f}^{\mathrm{b}}\left(p_{3}^{\prime}-p_{3}\right)_{\perp}}{\Delta_{3}-\left(p_{3 z}^{\prime}-p_{3 z}\right)+i \varepsilon} \times \\
& \times \frac{f_{d i f f}^{\mathrm{a}}\left(p_{2}^{\prime}-p_{2}\right)_{\perp}}{p_{m z}-p_{1 z}+\Delta_{0}+i \varepsilon} \cdot j^{\mu}\left(p_{1}+q, p_{1}\right) \cdot \Psi_{3_{\mathrm{He}}}\left(p_{1} ; p_{2} ; p_{3}\right) .
\end{aligned}
$$

It is convinient to rewrite variables of integration in eq.(97) according to the following definitions:

$$
\begin{aligned}
& \mathbf{k}_{3}=\mathbf{p}_{3}^{\prime}-\mathbf{p}_{3} \\
& \mathbf{k}_{2}=\mathbf{p}_{2}^{\prime}-\mathbf{p}_{2} \\
& \mathbf{k}_{2^{\prime} 3^{\prime}}=\left(\mathbf{p}_{2}^{\prime}-\mathbf{p}_{3}^{\prime}\right) / 2=-\mathbf{p}_{m} / 2-\mathbf{p}_{3}^{\prime} .
\end{aligned}
$$

(for the last one we replaced $p_{2}^{\prime}$ as given by eq.(86) and then used the expression for the missing momentum $\left.\mathbf{p}_{m} \equiv \mathbf{p}_{f}-\mathbf{q}=-\mathbf{p}_{r}-\mathbf{p}_{s}\right)$.

Old 3-dimensional momenta which were describing intermediate states of the ${ }^{3} \mathrm{He}$ break down reaction are connected to the new variables via set of relations

$$
\begin{aligned}
\mathbf{p}_{3}^{\prime} & =-\mathbf{k}_{2^{\prime} 3^{\prime}}-\mathbf{p}_{m} / 2, \\
\mathbf{p}_{2}^{\prime} & =2 \mathbf{k}_{2^{\prime} 3^{\prime}}+\mathbf{p}_{3}^{\prime}=\mathbf{k}_{2^{\prime} 3^{\prime}}-\mathbf{p}_{m} / 2 \\
\mathbf{p}_{3} & =\mathbf{p}_{3}^{\prime}-\mathbf{k}_{3}=-\mathbf{k}_{2^{\prime} 3^{\prime}}-\mathbf{k}_{3}-\mathbf{p}_{m} / 2 \\
\mathbf{p}_{2} & =\mathbf{p}_{2}^{\prime}-\mathbf{k}_{2}=\mathbf{k}_{2^{\prime} 3^{\prime}}-\mathbf{k}_{2}-\mathbf{p}_{m} / 2 \\
\mathbf{p}_{1} & =-\mathbf{p}_{2}-\mathbf{p}_{3}=\mathbf{k}_{2}+\mathbf{k}_{3}+\mathbf{p}_{m}
\end{aligned}
$$


With the help of this set eq.(97) reads as

$$
\begin{aligned}
A_{2 \mathrm{a}}^{\mu} & =\frac{F}{4} \int \frac{d^{3} k_{2^{\prime} 3^{\prime}}}{(2 \pi)^{3}} \frac{d^{3} k_{2}}{(2 \pi)^{3}} d^{3} k_{3} \Psi_{\mathbf{k}_{r s}}^{(+)}\left(k_{2^{\prime} 3^{\prime}}\right) \frac{f_{\text {diff }}^{\mathrm{b}}\left(k_{3 \perp}\right)}{-k_{3 z}+\Delta_{3}+i \varepsilon} \times \\
& \times \frac{f_{d i f f}^{\mathrm{a}}\left(k_{2 \perp}\right)}{-k_{2 z}-k_{3 z}+\Delta_{0}+i \varepsilon} \cdot j^{\mu}\left(k_{2}+k_{3}+p_{m}+q, k_{2}+k_{3}+p_{m}\right) \times \\
& \times \Psi_{{ }^{3} \mathrm{He}}\left(k_{2}+k_{3}+p_{m} ;-k_{2}+k_{2^{\prime} 3^{\prime}}-p_{m} / 2 ;-k_{2^{\prime} 3^{\prime}}-k_{3}-p_{m} / 2\right) .
\end{aligned}
$$

Similar to what we did in order to obtain eq.(42) for Impulse Approximation amplitude and then eq.(78) for Single Rescattering amplitude we can integrate over the plane wave part of the continuum wave function $\Psi_{\mathbf{k}_{r s}}\left(\mathbf{k}_{2^{\prime} 3^{\prime}}\right)$ ( the full form of which is given by eq.(41)). This reduces eq.(106) to

$$
\begin{aligned}
A_{2 \mathrm{a}}^{\mu(0)} & =\frac{F}{4} \int \frac{d k_{2 z} d^{2} k_{2 \perp}}{(2 \pi)^{3}} d k_{3 z} d^{2} k_{3 \perp} \frac{f_{d i f f}^{\mathrm{b}}\left(k_{3 \perp}\right)}{-k_{3 z}+\Delta_{3}+i \varepsilon} \times \\
& \times \frac{f_{d i f f}^{\mathrm{a}}\left(k_{2 \perp}\right)}{-k_{2 z}-k_{3 z}+\Delta_{0}+i \varepsilon} \cdot j^{\mu}\left(k_{2}+k_{3}+p_{m}+q, k_{2}+k_{3}+p_{m}\right) \times \\
& \times \Psi_{{ }^{\mathrm{He}}}\left(k_{2}+k_{3}+p_{m} ;-k_{2}+k_{r s}-p_{m} / 2 ;-k_{r s}-k_{3}-p_{m} / 2\right) .
\end{aligned}
$$

We will not consider "s" term of the Double Rescattering amplitude, which is corresponding to the diverging spherical wave part of the $\Psi_{\mathbf{k}_{r s}}^{(+)}$, since nine-fold integration makes it contribution negligible. Meanwhile, using relations similar to eq.(75) we can simplify eq.(107) a bit further. Keeping pole term only we first integrate over $d k_{3 z}$, and then over $d k_{2 z}$ again keeping pole term only. The result reads

$$
\begin{aligned}
A_{2 \mathrm{a}}^{\mu(\text { pole }, 0)} & =-\frac{F}{4} \int \frac{d^{2} k_{2 \perp} d^{2} k_{3 \perp}}{8 \pi} f_{d i f f}^{\mathrm{b}}\left(k_{3 \perp}\right) f_{d i f f}^{\mathrm{a}}\left(k_{2 \perp}\right) \times \\
& \times j^{\mu}\left(k_{2_{\left(\Delta_{0}-\Delta_{3}\right)}}+k_{3_{\left(\Delta_{3}\right)}}+p_{m}+q, k_{2_{\left(\Delta_{0}-\Delta_{3}\right)}}+k_{3_{\left(\Delta_{3}\right)}}+p_{m}\right) \times \\
& \times \Psi_{3 \mathrm{He}}\left(k_{2_{\left(\Delta_{0}-\Delta_{3}\right)}}+k_{3_{\left(\Delta_{3}\right)}}+p_{m} ;-k_{2_{\left(\Delta_{0}-\Delta_{3}\right)}}+k_{r} ;-k_{3_{\left(\Delta_{3}\right)}}+k_{s}\right)
\end{aligned}
$$


where $\mathbf{k}_{2_{\left(\Delta_{0}-\Delta_{3}\right)}}=\left(k_{2 \perp}, \Delta_{0}-\Delta_{3}\right)$ and $\mathbf{k}_{3\left(\Delta_{3}\right)}=\left(k_{3 \perp}, \Delta_{3}\right)$. We have also replaced

$$
\begin{aligned}
\mathbf{k}_{r s}-p_{m} / 2 & =\left(\mathbf{k}_{r}-\mathbf{k}_{s}\right) / 2+\left(\mathbf{k}_{r}+\mathbf{k}_{s}\right) / 2=\mathbf{k}_{r} \\
-\mathbf{k}_{r s}-p_{m} / 2 & =\left(-\mathbf{k}_{r}+\mathbf{k}_{s}\right) / 2+\left(\mathbf{k}_{r}+\mathbf{k}_{s}\right) / 2=\mathbf{k}_{s}
\end{aligned}
$$

To complete the calculation of double rescattering amplitude one should calculate also the amplitude $A_{\mathrm{b}}^{\mu}$ corresponding to the diagram of Figure 12(b). To do this we need interchange second and third arguments of the ${ }^{3} \mathrm{He}$ wave function as well as replace everywhere $p_{2}^{\prime} \leftrightarrow p_{3}^{\prime}$ and $p_{2} \leftrightarrow p_{3}$ in the eqs.(108). To preserve consistency, however, let us present the final result in the more general form, corresponding to eq.(106).

$$
\begin{aligned}
A_{2}^{\mu} & =A_{2 \mathrm{a}}^{\mu}+A_{2 \mathrm{~b}}^{\mu}=\frac{F}{4} \int \frac{d^{3} k_{2^{\prime} 3^{\prime}}}{(2 \pi)^{3}} \frac{d^{3} k_{2}}{(2 \pi)^{3}} d^{3} k_{3} \Psi_{\mathrm{k}_{r s}}^{(+)}\left(k_{2^{\prime} 3^{\prime}}\right) \times \\
& \times\left[\frac{f_{d i f f}^{\mathrm{b}}\left(k_{3 \perp}\right)}{-k_{3 z}+\Delta_{3}+i \varepsilon} \frac{f_{d i f f}^{\mathrm{a}}\left(k_{2 \perp}\right)}{-k_{2 z}-k_{3 z}+\Delta_{0}+i \varepsilon}+\right. \\
& \left.+\frac{f_{d i f f}^{\mathrm{a}}\left(k_{2 \perp}\right)}{-k_{2 z}+\Delta_{2}+i \varepsilon} \frac{f_{d i f f}^{\mathrm{b}}\left(k_{3 \perp}\right)}{-k_{2 z}-k_{3 z}+\Delta_{0}+i \varepsilon}\right] \times \\
& \times j^{\mu}\left(k_{2}+k_{3}+p_{m}+q, k_{2}+k_{3}+p_{m}\right) \times \\
& \times \Psi_{{ }^{\mathrm{He}}}\left(k_{2}+k_{3}+p_{m} ;-k_{2}+k_{2^{\prime} 3^{\prime}}-p_{m} / 2 ;-k_{2^{\prime} 3^{\prime}}-k_{3}-p_{m} / 2\right),
\end{aligned}
$$

where $\Delta_{2}=\frac{q_{0}}{q_{\mathrm{v}}}\left(E_{2}^{\prime}-E_{2}\right)$.

Though intuitively clear, it's still worth to point out that contrary to single rescattering case $p_{2} \leftrightarrow p_{3}, p_{2}^{\prime} \leftrightarrow p_{3}^{\prime}$ together with interchange of second and third arguments of the ${ }^{3} \mathrm{He}$ wave function leave $\Psi_{3 \mathrm{He}}$ invariant. Indeed since $k_{3^{\prime} 2^{\prime}}=-k_{2^{\prime} 3^{\prime}}$

$$
\begin{gathered}
-k_{2}+k_{2^{\prime} 3^{\prime}}-p_{m} / 2 ;-k_{2^{\prime} 3^{\prime}}-k_{3}-p_{m} / 2 \rightarrow-k_{3^{\prime} 2^{\prime}}-k_{2}-p_{m} / 2 ;-k_{3}+k_{3^{\prime} 2^{\prime}}-p_{m} / 2= \\
=-k_{2}+k_{2^{\prime} 3^{\prime}}-p_{m} / 2 ;-k_{2^{\prime} 3^{\prime}}-k_{3}-p_{m} / 2
\end{gathered}
$$




\section{Differential Cross Section}

\subsection{General Formula}

The (un-integrated) differential cross-section is equal to:

$$
\begin{aligned}
& d^{12} \sigma=\frac{1}{4 j^{3} \mathrm{He}}(2 \pi)^{4} \delta^{4}\left(k_{e}+P^{{ }^{3} \mathrm{He}}-k_{e}^{\prime}-p_{f}-p_{r}-p_{s}\right) \times \\
& \times\left.\frac{1}{4} \sum_{\text {nucleons spins }} \sum_{\mathcal{M}_{i}}\right|^{2} \frac{d^{3} k_{e}^{\prime}}{(2 \pi)^{3} 2 E_{e}^{\prime}} \frac{d^{3} p_{f}}{(2 \pi)^{3} 2 E_{f}} \frac{d^{3} p_{r}}{(2 \pi)^{3} 2 E_{r}} \frac{d^{3} p_{s}}{(2 \pi)^{3} 2 E_{s}},
\end{aligned}
$$

where $j_{3} \mathrm{He}=\sqrt{\left(k_{e} P_{{ }^{3} \mathrm{He}}\right)^{2}-m_{e}^{2} M_{3 \mathrm{He}}^{2}} / 3$. The factor $1 / 3$ in the flux factor $j_{3} \mathrm{He}$ reflects the fact that our ${ }^{3} \mathrm{He}$ wave function is normalized to one rather that to the number of nucleons, three.

In eq.(113) we sum over final and average over initial spins. The factor $1 / 4$ comes from the averaging over initial spins of the electron and the nucleus. Since one of the spectator nucleons (for example $p_{s}$ ) is not observed, one eliminates this degree of freedom by integrating over $d^{3} p_{s}$. Thus integrated differential cross section is

$$
\begin{aligned}
& d^{9} \sigma=\frac{1}{4 j_{3} \mathrm{He}}(2 \pi)^{4} \delta\left(E_{e}+M^{3} \mathrm{He}-E_{e}^{\prime}-E_{f}-E_{r}-E_{s}\right) \times
\end{aligned}
$$

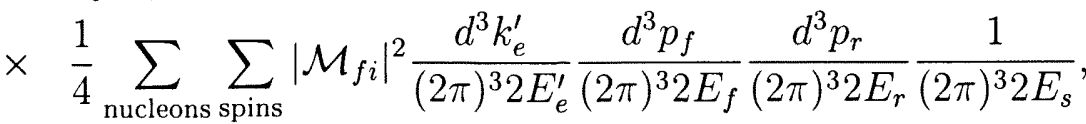

Delta-function integration provides us with

$$
\mathbf{p}_{s}=\mathbf{k}_{e}-\mathbf{k}_{\mathbf{e}}^{\prime}-\mathbf{p}_{f}-\mathbf{p}_{r}
$$

The transition matrix $\mathcal{M}_{f i}$ represents the convolution of the electron and nuclear currents, in which the nuclear current given by sum of the PWIA term (eq.(42)), pair 
distortion amplitude ( eq.(43) ), as well as single ( eq.(84)) and double rescattering amplitudes ( eq.(111)):

$$
\mathcal{M}_{f i}=-4 \pi \alpha \frac{1}{q^{2}} j_{\mu}^{(e)} \cdot\left(A_{0}^{\mu}+A_{0 s}^{\mu}+A_{1}^{\mu}+A_{2}^{\mu}\right)
$$

Let us consider now electromagnetic amplitude $j^{\mu}$. According to eq.(57) the char-

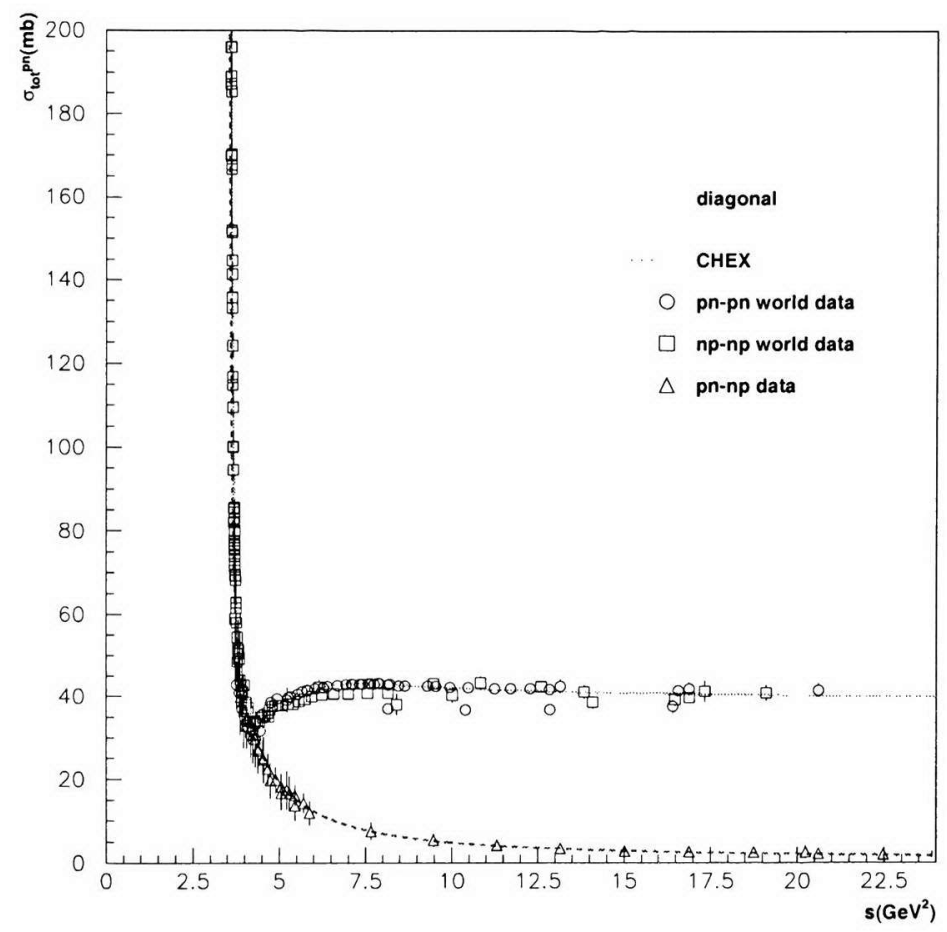

Figure 13: Total cross section for proton-neutron charge-exchange scattering (dashed line) compared with diagonal scattering total cross section.

acteristic average momenta transferred during rescattering are $\propto 1 / B$. This fact together with the kinematic restrictions of eq.(2) allows us to assume $q \gg p_{m}, k_{1}, k_{2}$. Due to the high momentum transfer it is also safe to assume that charge-exchange 
effects are negligible during reinteractions of the leading nucleon. For laboratory momenta starting from $1 \mathrm{GeV} / \mathrm{c}$ total cross section of charge-exchange $N N$ interactions drops rapidly, as it is depicted at the Figure 13.

Therefore one can factorize electromagnetic currents in eq.(84) and eq.(111) approximating

$$
\left.\begin{array}{l}
j^{\mu}\left(k+p_{m}+q, k+p_{m}\right) \\
j^{\mu}\left(k_{2}+k_{3}+p_{m}+q, k_{2}+k_{3}+p_{m}\right)
\end{array}\right\} \approx j^{\mu}\left(p_{m}+q, p_{m}\right) .
$$

Recalling that $j^{\mu}\left(p_{m}+q, p_{m}\right) \equiv \bar{u}\left(p_{f}, s_{f}\right) \Gamma_{\gamma^{*} N}^{\mu} u\left(p_{m}, s\right)$ we may present transition matrix $\mathcal{M}_{f i}$ as follows:

$$
\begin{aligned}
\mathcal{M}_{f i} & =4 \pi \alpha \frac{1}{q^{2}} j_{\mu}^{(e)} \sum_{s} \bar{u}\left(p_{f}, s_{f}\right) \Gamma_{\gamma^{*} N}^{\mu} u\left(p_{m}, s\right) F\left\{(2 \pi)^{3} \Psi_{3_{\mathrm{He}}}\left(p_{m}, s ; p_{r} ; p_{s}\right)+\right. \\
& +4 \pi \int d^{3} k_{23} \frac{f_{N N}^{\mathrm{off}} \text { shell }\left(\mathbf{k}_{r s}, \mathbf{k}_{23}\right)}{k_{23}^{2}-k_{r s}^{2}-i \varepsilon} \cdot \Psi_{3_{\mathrm{He}}}\left(p_{m}, s ; k_{23}-p_{m} / 2 ;-k_{23}-p_{m} / 2\right)- \\
& -\frac{1}{2} \int \frac{d^{3} k}{(2 \pi)^{3}} d^{3} k_{23}^{\prime} \Psi_{\mathbf{k}_{r s}}^{(+)}\left(k_{23}^{\prime}\right) \times \\
& \times\left[\frac{f_{d i f f}^{\mathrm{a}}\left(k_{\perp}\right)}{-k_{z}+\Delta_{0}+i \varepsilon} \Psi_{3_{\mathrm{He}}}\left(k+p_{m}, s ;-k+k_{23}^{\prime}-p_{m} / 2 ;-k_{23}^{\prime}-p_{m} / 2\right)+\right. \\
& \left.+\frac{f_{d i f f}^{\mathrm{b}}\left(k_{\perp}\right)}{-k_{z}+\Delta_{0}+i \varepsilon} \Psi_{3 \mathrm{He}}\left(k+p_{m}, s ; k_{23}^{\prime}-p_{m} / 2 ;-k-k_{23}^{\prime}-p_{m} / 2\right)\right]+ \\
& +\frac{1}{4} \int \frac{d^{3} k_{2^{\prime} 3^{\prime}}}{(2 \pi)^{3}} \frac{d^{3} k_{2}}{(2 \pi)^{3}} d^{3} k_{3} \Psi_{\mathbf{k}_{r s}}^{(+)}\left(k_{2^{\prime} 3^{\prime}}\right) \times \\
& \times\left[\frac{f_{d i f f}^{\mathrm{b}}\left(k_{3 \perp}\right)}{-k_{3 z}+\Delta_{3}+i \varepsilon} \frac{f_{d i f f}^{\mathrm{a}}\left(k_{2 \perp}\right)}{-k_{2 z}-k_{3 z}+\Delta_{0}+i \varepsilon}+\right. \\
& \left.+\frac{f_{d i f f}^{\mathrm{a}}\left(k_{2 \perp}\right)}{-k_{2 z}+\Delta_{2}+i \varepsilon} \frac{f_{d i f f}^{\mathrm{b}}\left(k_{3 \perp}\right)}{-k_{2 z}-k_{3 z}+\Delta_{0}+i \varepsilon}\right] \times \\
& \left.\times \Psi_{3 \mathrm{He}}\left(k_{2}+k_{3}+p_{m}, s ;-k_{2}+k_{2^{\prime} 3^{\prime}}-p_{m} / 2 ;-k_{2^{\prime} 3^{\prime}}-k_{3}-p_{m} / 2\right)\right\}
\end{aligned}
$$

In order to find $\left|\mathcal{M}_{f i}\right|^{2}$ from eq.(118) and it's average over spin indices let us consider first the electromagnetic matrix elements of interacting electron and nucleon 
averaged over corresponding spin variables. Namely, one can write

$$
\begin{aligned}
& \sum_{s_{e}, s e^{\prime}} j_{\mu}^{(e)}\left(j_{\nu}^{(e)}\right)^{*} \sum_{s_{f}} \bar{u}\left(p_{f}, s_{f}\right) \Gamma_{\gamma^{*} N}^{\mu} u\left(p_{m}, s\right) \bar{u}\left(p_{m}, s^{\prime}\right) \Gamma_{\gamma^{*} N}^{\nu} u\left(p_{f}, s_{f}\right)= \\
& l_{\mu \nu} \sum_{s_{f}} \bar{u}\left(p_{f}, s_{f}\right) \Gamma_{\gamma^{*} N}^{\mu} u\left(p_{m}, s\right) \bar{u}\left(p_{m}, s^{\prime}\right) \Gamma_{\gamma^{*} N}^{\nu} u\left(p_{f}, s_{f}\right)
\end{aligned}
$$

where $l_{\mu \nu}=4\left(\left(k_{e}\right)_{\mu}\left(k_{e}^{\prime}\right)_{\nu}+\left(k_{e}\right)_{\nu}\left(k_{e}^{\prime}\right)_{\mu}-g_{\mu \nu} k_{e} \cdot k_{e}^{\prime}\right)$ is the usual lepton tensor.

It was explicitly shown in [14] that only terms with $s=s^{\prime}$ survive in eq. (119) upon contraction with the symmetric tensor $l_{\mu \nu}$. Therefore we can write

$$
\begin{aligned}
& l_{\mu \nu} \sum_{s_{f}} \bar{u}\left(p_{f}, s_{f}\right) \Gamma_{\gamma^{*} N}^{\mu} u\left(p_{m}, s\right) \bar{u}\left(p_{m}, s^{\prime}\right) \Gamma_{\gamma^{*} N}^{\nu} u\left(p_{f}, s_{f}\right)= \\
& l_{\mu \nu} \sum_{s_{f}} \bar{u}\left(p_{f}, s_{f}\right) \Gamma_{\gamma^{*} N}^{\mu} u\left(p_{m}, s\right) \bar{u}\left(p_{m}, s\right) \Gamma_{\gamma^{*} N}^{\nu} u\left(p_{f}, s_{f}\right) \delta_{s, s^{\prime}}= \\
& l_{\mu \nu} \frac{1}{2} w^{\mu \nu} \delta_{s, s^{\prime}}
\end{aligned}
$$

where $w^{\mu \nu}$ is the spin-averaged hadronic tensor which is discussed in the Appendix C.

Using Eqs. (119) and (120) for the squared absolute value of transition matrix elements given by eq. (118) we can rewrite eq. (114) for the differential cross section as follows

$$
\begin{aligned}
\frac{d^{9} \sigma}{d^{3} k_{e}^{\prime} d^{3} p_{f} d^{3} p_{r}} & =\frac{1}{4 j_{3} \mathrm{He}}(2 \pi)^{4} \delta\left(E_{e}+M_{3_{\mathrm{He}}}-E_{e}^{\prime}-E_{f}-E_{r}-E_{s}\right) \times \\
& \times \frac{Z}{4}\left(4 \pi \alpha \frac{1}{q^{2}}\right)^{2} l_{\mu \nu} \frac{1}{2} w^{\mu \nu} \sum_{\text {nucleons spins }} \sum_{\mid} \mid F\left\{(2 \pi)^{3} \Psi_{3_{\mathrm{He}}}\left(p_{m} ; p_{r} ; p_{s}\right)+\right. \\
& +4 \pi \int d^{3} k_{23} \frac{f_{N N}^{\text {off shell }}\left(\mathbf{k}_{r s}, \mathbf{k}_{23}\right)}{k_{23}^{2}-k_{r s}^{2}-i \varepsilon} \cdot \Psi_{3_{\mathrm{He}}}\left(p_{m} ; k_{23}-p_{m} / 2 ;-k_{23}-p_{m} / 2\right)- \\
& -\frac{1}{2} \int \frac{d^{3} k}{(2 \pi)^{3}} d^{3} k_{23}^{\prime} \Psi_{\mathbf{k}_{r s}}^{(+)}\left(k_{23}^{\prime}\right) \times \\
& \times\left[\frac{f_{d i f f}^{\mathrm{a}}\left(k_{\perp}\right)}{-k_{z}+\Delta_{0}+i \varepsilon} \Psi_{{ }_{3 \mathrm{He}}}\left(k+p_{m} ;-k+k_{23}^{\prime}-p_{m} / 2 ;-k_{23}^{\prime}-p_{m} / 2\right)+\right.
\end{aligned}
$$




$$
\begin{aligned}
& \left.+\frac{f_{d i f f}^{\mathrm{b}}\left(k_{\perp}\right)}{-k_{z}+\Delta_{0}+i \varepsilon} \Psi_{3 \mathrm{He}}\left(k+p_{m} ; k_{23}^{\prime}-p_{m} / 2 ;-k-k_{23}^{\prime}-p_{m} / 2\right)\right]+ \\
& +\frac{1}{4} \int \frac{d^{3} k_{2^{\prime} 3^{\prime}}}{(2 \pi)^{3}} \frac{d^{3} k_{2}}{(2 \pi)^{3}} d^{3} k_{3} \Psi_{\mathbf{k}_{r s}}^{(+)}\left(k_{2^{\prime} 3^{\prime}}\right) \times \\
& \times\left[\frac{f_{d i f f}^{\mathrm{b}}\left(k_{3 \perp}\right)}{-k_{3 z}+\Delta_{3}+i \varepsilon} \frac{f_{d i f f}^{\mathrm{a}}\left(k_{2 \perp}\right)}{-k_{2 z}-k_{3 z}+\Delta_{0}+i \varepsilon}+\right. \\
& \left.+\frac{f_{d i f f}^{\mathrm{a}}\left(k_{2 \perp}\right)}{-k_{2 z}+\Delta_{2}+i \varepsilon} \frac{f_{d i f f}^{\mathrm{b}}\left(k_{3 \perp}\right)}{-k_{2 z}-k_{3 z}+\Delta_{0}+i \varepsilon}\right] \times \\
& \left.\times \Psi_{3_{\mathrm{He}}}\left(k_{2}+k_{3}+p_{m} ;-k_{2}+k_{2^{\prime} 3^{\prime}}-p_{m} / 2 ;-k_{2^{\prime} 3^{\prime}}-k_{3}-p_{m} / 2\right)\right\}\left.\right|^{2} \times \\
& \times \frac{1}{(2 \pi)^{3} 2 E_{e}^{\prime}} \frac{1}{(2 \pi)^{3} 2 E_{f}} \frac{1}{(2 \pi)^{3} 2 E_{r}} \frac{1}{(2 \pi)^{3} 2 E_{s}} .
\end{aligned}
$$

\subsection{Nuclear Decay Function}

Generalizing the spectral function formalism of the semi-inclusive $\left(e, e^{\prime} N\right)$ reaction for the exclusive reaction of eq.(1) we introduce the nuclear Decay function $D$ which in the IA framework characterizes the joint probability to find a nucleon in the nuclear ground state function with momentum $\mathbf{p}_{m}$ (and missing energy $E_{m}$ ) and the recoil nucleon with momentum $\mathbf{p}_{r}$ in the decay product of residual $A-1$ nucleus. Within IA the cross section of reaction eq.(1) can be represented as follows:

$$
\frac{d^{9} \sigma}{d^{3} k_{e}^{\prime} d^{3} p_{f} d^{3} p_{r}}=\frac{j_{N}}{j^{3} \mathrm{He}} K \cdot Z \sigma_{e N}\left(p_{f}, k_{e}, k_{e}^{\prime}\right) \cdot D_{\mathrm{IA}}\left(\mathbf{p}_{m}, E_{m}, \mathbf{p}_{r}\right)
$$

where $E_{m}=T_{r}+T_{s}+\left|\epsilon_{3 \mathrm{He}}\right|-p_{m}^{2} / 4 m^{2}$. The factor $\sigma_{e N}\left(k_{e}, k_{e}^{\prime}, p_{f}\right)$ is defined as

$$
\sigma_{e N}\left(k_{e}, k_{e}^{\prime}, p_{f}\right)=\frac{F}{4 j_{N}}\left(4 \pi \alpha \frac{1}{q^{2}}\right)^{2} \frac{1}{4} l_{\mu \nu} w^{\mu \nu} \frac{1}{(2 \pi)^{3} 2 E_{e}^{\prime}} \frac{1}{(2 \pi)^{3} 2 E_{f}} \frac{1}{(2 \pi)^{3} 2 E_{r}} \frac{1}{(2 \pi)^{3} 2 E_{s}} .
$$

If we keep in eq.(121) only IA term given by eq.(40) then $D_{\mathrm{IA}}$ is equal to

$$
D_{\mathrm{IA}}\left(\mathbf{p}_{m}, E_{m}, \mathbf{p}_{r}\right)=\frac{1}{2} \sum_{s_{A}, s_{m}, s_{r} \cdot s_{s}} \mid \sum_{s_{2}, s_{3}} \int d^{3} k_{23} \Psi_{\mathbf{k}_{r s}}^{(+)}\left(k_{23}, s_{2}, s_{3}\right) \times
$$




$$
\begin{aligned}
& \times\left.\quad \Psi_{3_{\mathrm{He}}}\left(p_{m}, s_{m} ; k_{23}-p_{m} / 2, s_{2} ;-k_{23}-p_{m} / 2, s_{3}\right)\right|^{2} \times \\
& \times \delta\left(E_{m}-T_{r}-T_{s}-\left|\epsilon_{3 \mathrm{He}}\right|+p_{m}^{2} / 4 m^{2}\right) .
\end{aligned}
$$

The Decay function as it defined in eq.(124) is normalized as follows

$$
\begin{aligned}
& \int d^{3} p_{r} D_{\mathrm{IA}}\left(\mathbf{p}_{m}, E_{m}, \mathbf{p}_{r}\right)=S\left(E_{m}, \mathbf{p}_{m}\right), \\
& \int d E_{m} d^{3} p_{m} S\left(E_{m}, \mathbf{p}_{m}\right)=1 .
\end{aligned}
$$

We can generalize the definition of the Decay function to include final state interactions. Such a generalization usually meaningful within approximation in which electromagnetic current is factorized from rescattering integrals in eq.(84) and eq.(111) (c.f. eq.(117)). This factorization results to the formula similar to eq.(122) with $D_{\text {IA }}$ replaced by distorted wave impulse approximation (DWIA) Decay function $D_{\text {DwIA }}$. Based on eqs.(42), (43), (84) and (111) one obtains:

$$
\begin{aligned}
& D_{\text {DWIA }}\left(Q^{2}, \mathbf{q}, \mathbf{p}_{m}, E_{m}, \mathbf{p}_{r}\right)=\frac{1}{2} \sum_{s_{A}, s_{m}, s_{r}, s_{s}} \mid \sum_{s_{2}, s_{3}} \int d^{3} k_{23} \Psi_{\mathbf{k}_{r s}}^{(+)}\left(k_{23}, s_{2}, s_{3}\right) \times \\
& \times \Psi_{3 \mathrm{He}}\left(p_{m}, s_{m} ; k_{23}-p_{m} / 2, s_{2} ;-k_{23}-p_{m} / 2, s_{3}\right)+\sum_{s_{1}, s_{2}, s_{3}} \frac{-1}{2} \int \frac{d^{3} k}{(2 \pi)^{3}} d^{3} k_{23}^{\prime} \times \\
& \times \Psi_{\mathbf{k}_{r s}}^{(+)}\left(k_{23}^{\prime}\right) \cdot\left[\frac{f_{d i f f}^{\mathrm{a}}\left(k_{\perp}\right)}{-k_{z}+\Delta_{0}+i \varepsilon} \Psi_{{ }^{\mathrm{He}}}\left(k+p_{m} ;-k+k_{23}^{\prime}-p_{m} / 2 ;-k_{23}^{\prime}-p_{m} / 2\right)+\right. \\
& \left.+\frac{f_{d i f f}^{\mathrm{b}}\left(k_{\perp}\right)}{-k_{z}+\Delta_{0}+i \varepsilon} \Psi_{3 \mathrm{He}}\left(k+p_{m} ; k_{23}^{\prime}-p_{m} / 2 ;-k-k_{23}^{\prime}-p_{m} / 2\right)\right]+ \\
& +\frac{1}{4} \int \frac{d^{3} k_{2^{\prime} 3^{\prime}}}{(2 \pi)^{3}} \frac{d^{3} k_{2}}{(2 \pi)^{3}} d^{3} k_{3} \Psi_{\mathbf{k}_{r s}}^{(+)}\left(k_{2^{\prime} 3^{\prime}}\right) \cdot\left[\frac{f_{d i f f}^{\mathrm{b}}\left(k_{3 \perp}\right)}{-k_{3 z}+\Delta_{3}+i \varepsilon} \frac{f_{d i f f}^{\mathrm{a}}\left(k_{2 \perp}\right)}{-k_{2 z}-k_{3 z}+\Delta_{0}+i \varepsilon}+\right. \\
& \left.+\frac{f_{\text {diff }}^{\mathrm{a}}\left(k_{2 \perp}\right)}{-k_{2 z}+\Delta_{2}+i \varepsilon} \frac{f_{\text {diff }}^{\mathrm{b}}\left(k_{3 \perp}\right)}{-k_{2 z}-k_{3 z}+\Delta_{0}+i \varepsilon}\right] \times \\
& \left.\times \Psi_{{ }^{3} \mathrm{He}}\left(k_{2}+k_{3}+p_{m} ;-k_{2}+k_{2^{\prime} 3^{\prime}}-p_{m} / 2 ;-k_{2^{\prime} 3^{\prime}}-k_{3}-p_{m} / 2\right)\right\}\left.\right|^{2} \times \\
& \times \delta\left(E_{m}-T_{r}-T_{s}-\left|\epsilon_{3 \mathrm{He}}\right|+p_{m}^{2} / 4 m^{2}\right) .
\end{aligned}
$$




\section{$9 \quad$ Numerical Estimates}

\subsection{Subroutine eheppn}

In this section we present numerical estimates of the calculations carried out in the previous sections. The Fortran90 external subroutine eheppn written by Misak Sargsian for calculation of the PWIA cross section for the ${ }^{3} \mathrm{He}\left(e, e^{\prime} N N\right) N$ reaction has been significantly upgraded in order to account for Final State Interaction effects, including spectator pair distortion, single rescattering of the knocked out nucleon and double rescattering. Transition matrix elements were programmed based on the formula of eq.(118). For $\Psi_{3 \mathrm{He}}$ we have used exact Faddeév ${ }^{3} \mathrm{He}$ wave function from the Bochum group [15] which takes into account 102 components of ${ }^{3} \mathrm{He}$ WF. This state of the art solution uses different realistic $N N$ and $N N N$ potentials. One of the available $N N$ potentials is Argonne v18, which has a dominant charge-independent piece plus additional charge-dependent and charge-symmetry-breaking terms (total of 18 terms), including a complete electromagnetic interaction. Another potential is the high-precision, charge-dependent Bonn nucleon-nucleon potential (CD-Bonn). In addition to these, the Bochum group also uses Urbana IX three-nucleon interactions. The description of the general approach to the exact solution of three-nucleon bound systems can be found in the Appendix A.

The spectator pair soft scattering amplitude, which is given by eq.(49), has been computed using the subroutine NNSOLA, courtesy of Virginia Tech Partial-Wave Analysis Group (SAID). The parametrization of hard rescattering amplitudes $f_{\text {diff }}$ 
is given by eq.(57). All the relevant parameters have been deduced from the world data for $N N$ scatterings (see Appendix D for Fortran code).

After delta function integration of the eq.(121) we left with the eight-fold cross section differential in $\left\{E_{e}, \Omega_{e},\left|\mathbf{p}_{\mathbf{f}}\right|, \Omega_{f}, \Omega_{r}\right\}$ where $E_{e}, \Omega_{e}$ are the energy and solid angel of the outgoing electron, $p_{f}$ and $\Omega_{f}$ are the momentum and solid angle of the struck nucleon, and $\Omega_{r}$ is the solid angle of the second nucleon. From these 8 variables we can calculate remaining kinematical quantities. The cross section is calculated separately for each combination of indexes $(f, r, s)=(p p n),(p n p),(n p p)$.

\subsection{Comparison with Experiment}

To date, there have been only few measurements of $\left(e, e^{\prime} n p\right)$ or $\left(e, e^{\prime} p p\right)$ two nucleon knockouts from nuclei. We compare our three-body break up calculations with the data of ${ }^{3} \mathrm{He}\left(e, e^{\prime} p p\right) n$ experiment performed in Hall B at the Thomas Jefferson National Accelerator Facility by Niyazov et al. [17], [16]. These measurements were part of the "e2" run group that took data in Spring 1999 and they represent the first measurements of two nucleon momentum distributions in ${ }^{3} \mathrm{He}$.

Reference [17] reports $2.2 \mathrm{GeV}$ and $4.4 \mathrm{GeV}$ electron scattering measurements from ${ }^{3} \mathrm{He}$ using a $100 \%$ duty factor beam at currents between 5 and $10 \mathrm{nA}$ incident on a $4.1-\mathrm{cm}$ long liquid ${ }^{3} \mathrm{He}$ target. Almost all outgoing charged particles have been detected in the Jefferson Lab CLAS (CEBAF Large Acceptance Spectrometer), a nearly $4 \pi$ magnetic spectrometer. Momentum coverage of CLAS extends down to $0.25 \mathrm{GeV} / \mathrm{c}$ for protons over a polar angular range of $8^{\circ}<\theta<140^{\circ}$ while spanning 
nearly $80 \%$ of the azimuth. Software fiducial cuts exclude regions of non-uniform detector response, while acceptance and tracking efficiencies are estimated using GSIM, the CLAS GEANT Monte-Carlo simulation. It is important to point out that in our Monte-Carlo simulations we have applied only kinematical acceptances of the experiment. No detector acceptances were taken into account.

The experiment kinematics was restricted by the following requirements:

- $p_{n} \geq 250 \mathrm{MeV} / \mathrm{c}$.

Since the threshold for proton detection is $p_{p}=250 \mathrm{MeV} / \mathrm{c}$ cut on the neutron momentum $p_{n} \geq 250 \mathrm{MeV} / \mathrm{c}$ has been applied. This effectively suppresses the events where two protons share the energy transfer due to the hard final state rescattering and the neutron is low momentum spectator.

- $T_{r, s} \leq q_{0} * 0.2$.

Since experiment was dedicated to deduce spectator correlated pairs (which we call $r$ and $s$ nucleons) $T_{r, s} \leq q_{0} * 0.2$ cuts have been applied, where $T$ stays for kinetic energy and $q_{0}$ for the energy transfer. This is corresponding to the events where $r$ and $s$ nucleons each have less than $20 \%$ of the energy transfer and leading $f$ nucleon has the remainder. The opening angle of the spectators after these cuts shows a peak at 180 degrees, which is a strong indication of correlated $N N$ pairs.

- $p_{f}^{\perp} \leq 0.3 \mathrm{GeV} / \mathrm{c}$.

In order to reduce further the effects of final state rescattering cut on the 
perpendicular component (relative to $\mathbf{q}$ ) of the struck nucleon has been applied. Almost all the resulting pairs are now back-to-back.

After all these cuts we have predominantly spectator pairs and measured momentum distributions reflect the pair's initial momentum distribution in the nucleus.

In the Figures 14 and 16 we compare our calculations with the data for relative $p_{\text {rel }}$ and total $p_{t o t}$ momentum distributions of the $p n$ and $p p$ pairs for beam energies $2.2 \mathrm{GeV}$ and $4.4 \mathrm{GeV}$. For $E_{e}=2.2 \mathrm{GeV} 4$-momentum transfer $Q^{2}$ concentrated in the range $0.5 \leq Q^{2} \leq 1.0 \mathrm{GeV}^{2}$. For $E_{e}=4.4 \mathrm{GeV}$ the range was $1.0 \leq Q^{2} \leq 2.0 \mathrm{GeV}^{2}$. As usually, we define

$$
\begin{aligned}
& p_{\text {rel }}=\left|\mathbf{p}_{r}-\mathbf{p}_{s}\right| / 2, \\
& p_{\text {tot }}=\left|\mathbf{p}_{r}+\mathbf{p}_{s}\right|
\end{aligned}
$$

Short range correlated pairs must have large relative momentum and small total momentum.

All the quantities and cross sections are given in the lab frame. Cross sections on the plots given in the arbitrary units.

For experimental data the relative momentum distribution rises rapidly starting at about $0.25 \mathrm{GeV} / \mathrm{c}$ (limited by the minimum nucleon momenta of $0.25 \mathrm{GeV} / \mathrm{c}$ ), peaks at about $0.35 \mathrm{GeV} / \mathrm{c}$, and has a tail extending to about $0.7 \mathrm{GeV} / \mathrm{c}$. The total momentum distribution rises rapidly from 0 , peaks at about $0.25 \mathrm{GeV} / \mathrm{c}$, and falls rapidly. The momentum distributions have an upper limit determined by the cut $T_{r, s}<0.2 * q_{0}$. 
Our Monte-Carlo simulations produce reasonable qualitative agreement with the data for $p n$ pairs (see Figures $14 \mathrm{a}$ ) and b) ). PWIA amplitude calculations represented by the solid histograms, PWIA + pair distortion - by the dashed line. The filled histograms include in addition the effects of single rescatterings. Double rescattering contribution in this kinematics estimated to be about $10 \%$. This contribution is not depicted on the current plots. For $p n$ pairs the normalization uncertainties (systematic uncertainties) of the data are $16 \%$ for $2.2 \mathrm{GeV}$ and $22 \%$ for $4.4 \mathrm{GeV}$ (see [17]).

For $p p$ pairs systematic uncertainties are $16 \%$ for $2.2 \mathrm{GeV}$ data and $34 \%$ for $4.4 \mathrm{GeV}$. We observe that $p p$ situation looks less satisfactory. One should bear in mind that we didn't reconstruct our events by CLAS event reconstruction package. It is also should be pointed out that the available statistics of experimental data after all the cuts are not very high. For $4.4 \mathrm{GeV}$ there are only about $340 p n$ pairs and $110 p p$ pairs (and ten times more events for $2.2 \mathrm{GeV}$ ). For $p p$ relative momentum distribution our peak shifted towards smaller momenta. However independent calculations by J.M. Laget presented in [16] (see Figure 15) favor our distribution rather than data. For $p p$ total momentum our calculations show discrepancy both with Laget calculations and available data. Same Hall B group took more measurements during new 2001 run, and we are looking forward for additional data. 

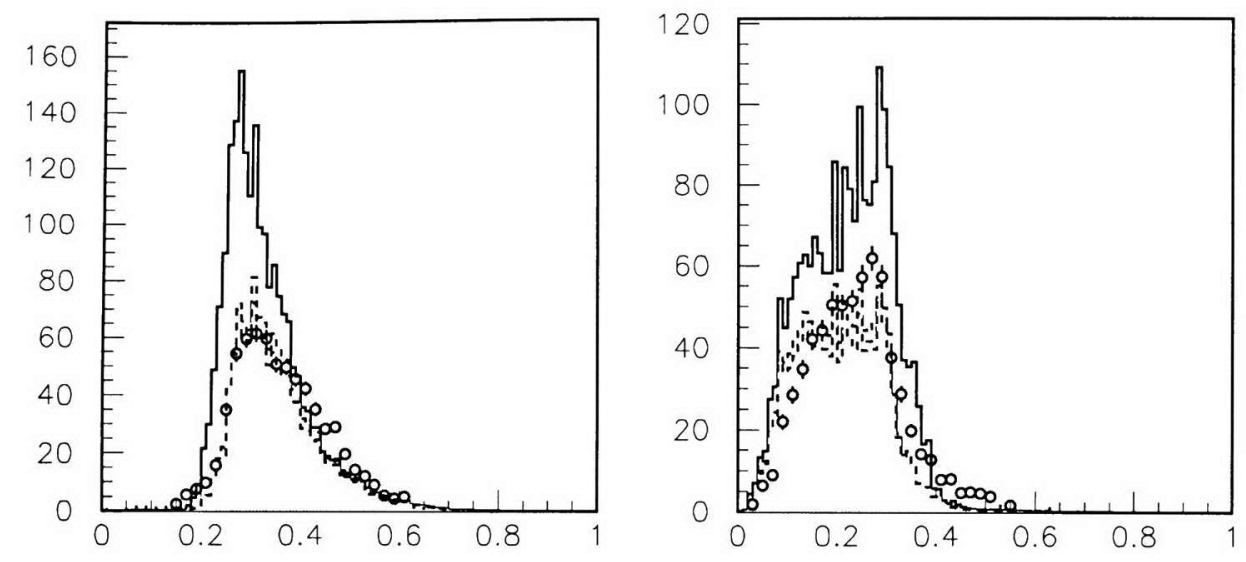

a) $p_{\text {rel }}(G e V / c)$, pn-pairs

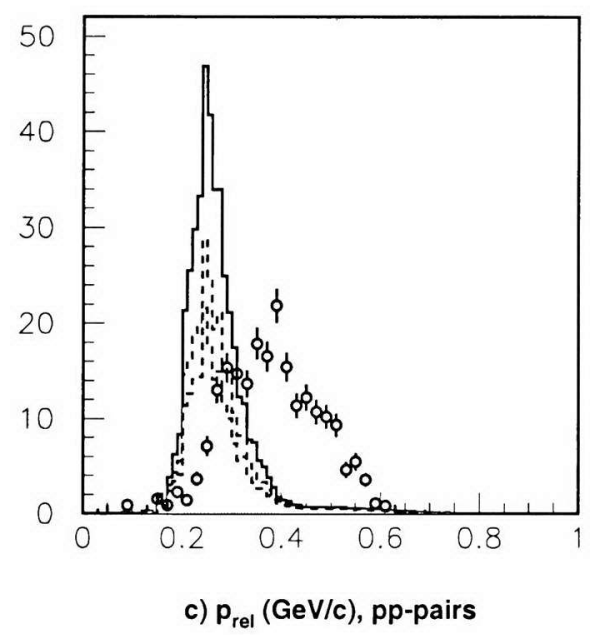

b) $p_{\text {tot }}(\mathrm{GeV} / \mathrm{c})$, pn-pairs

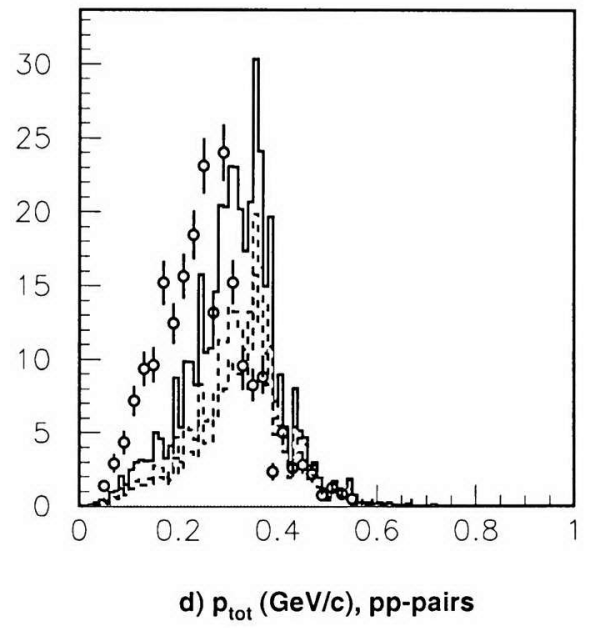

Figure 14: Cross section (with arbitrary normalization) vs relative momentum and total momentum of NN spectator pairs at $2.2 \mathrm{GeV}$. Points = data, solid histograms = PWIA amplitude calculations, dashed histograms = PWIA + pair distortion, filled histograms $=$ PWIA + pair distortion+single rescattering. a) pn pair relative momentum b) $p n$ total momentum c) $p p$ relative momentum d) $p p$ total momentum. 


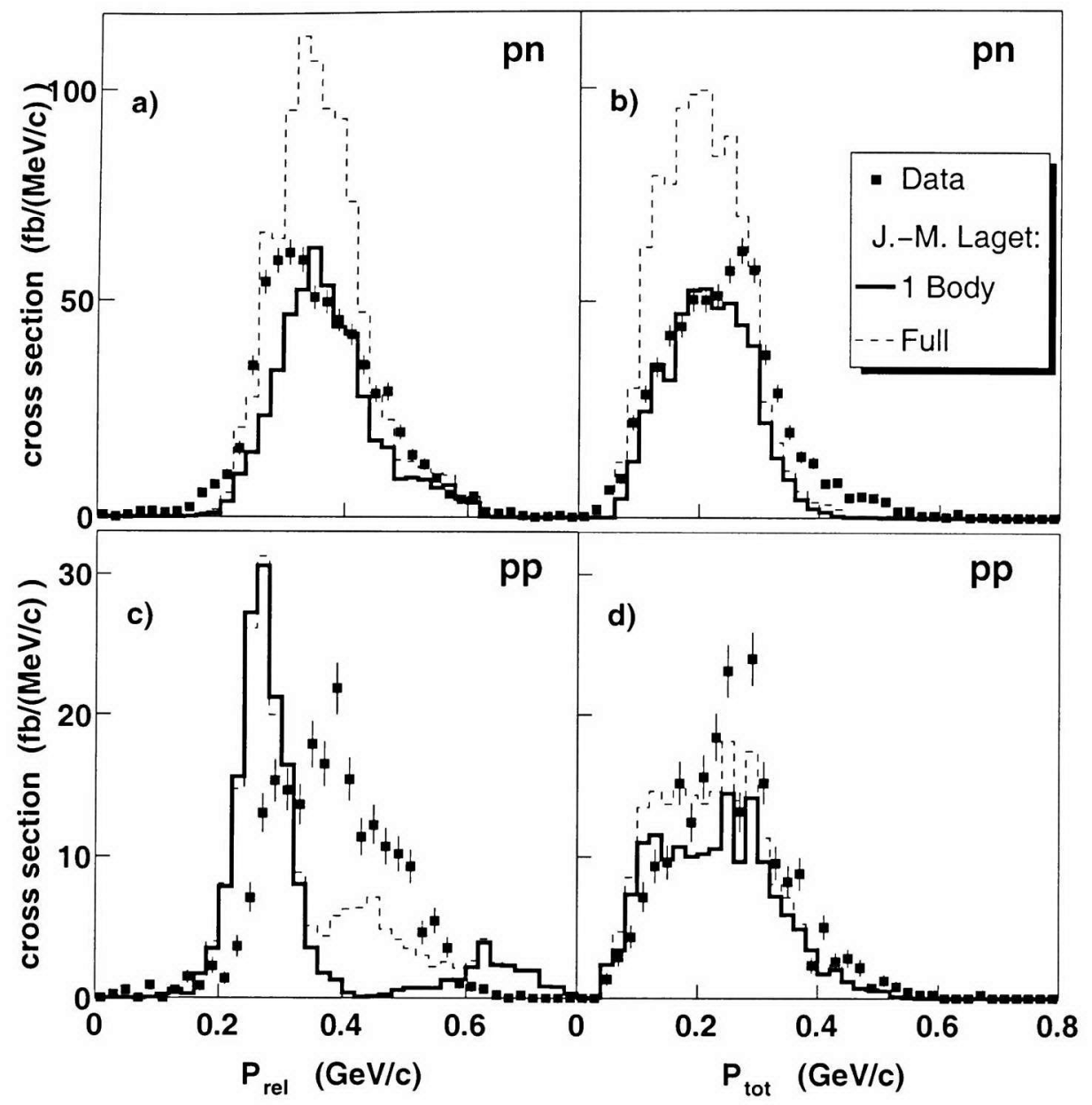

Figure 15: Cross section vs relative momentum and total momentum of NN spectator pairs at $2.2 \mathrm{GeV}$ (Ref. [16]). Points = data, solid histogram = J.-M. Laget onebody calculations, dashed histogram = Laget's full calculations (diagrams with no spectator nucleons). a) $p n$ pair relative momentum b) $p n$ total momentum c) $p p$ relative momentum d) $p p$ total momentum. 

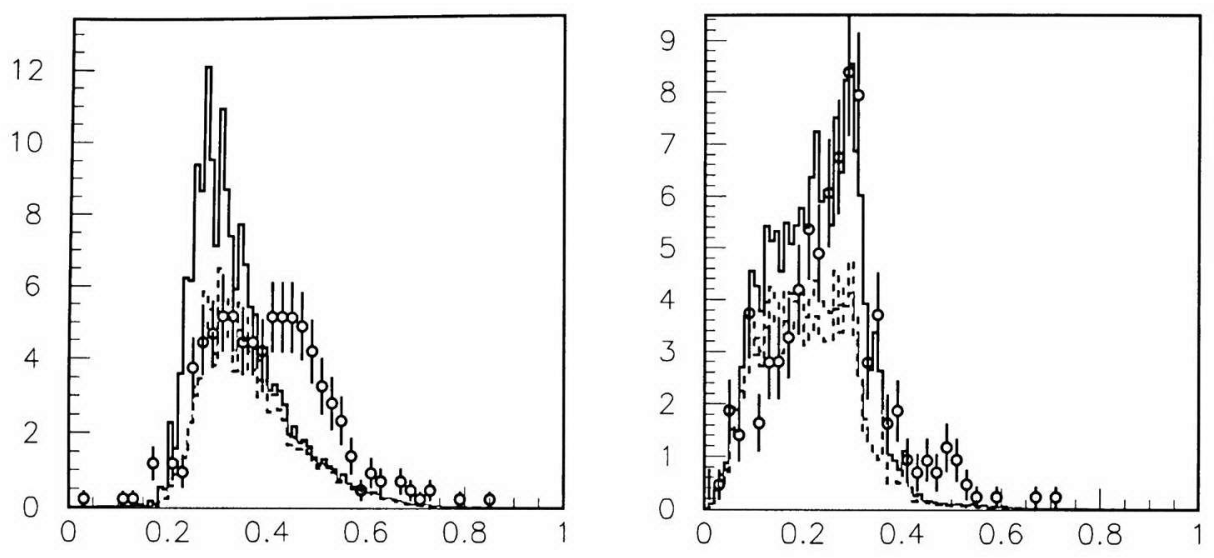

a) $p_{\text {rel }}(\mathrm{GeV} / \mathrm{c})$, pn-pairs

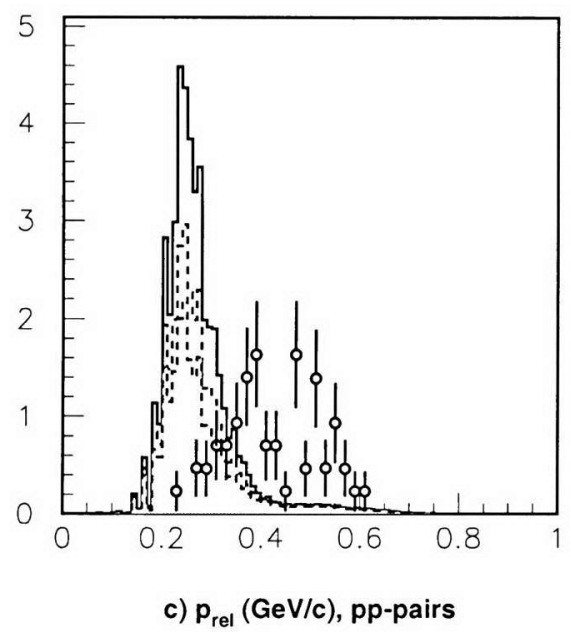

b) $p_{\text {tot }}(\mathrm{GeV} / \mathrm{c})$, pn-pairs

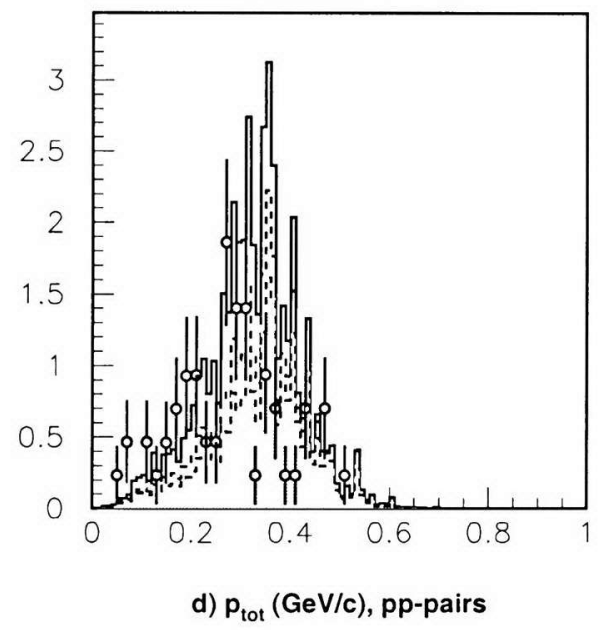

Figure 16: Cross section (with arbitrary normalization) vs relative momentum and total momentum of NN spectator pairs at $4.4 \mathrm{GeV}$. Points $=$ data, solid histograms = PWIA amplitude calculations, dashed histograms = PWIA + pair distortion, filled histograms $=$ PWIA + pair distortion + single rescattering. a) $p n$ pair relative momentum b) $p n$ total momentum c) $p p$ relative momentum d) $p p$ total momentum. 


\section{Conclusions}

We have developed a theory of high-energy exclusive electrodisintegration of three nucleon system on the example of ${ }^{3} \mathrm{He}\left(e, e^{\prime} N N\right) N$ reaction. Derivations done in kinematics where one nucleon in final state can be clearly identified as a knockedout nucleon that carries practically whole momentum of the virtual photon.

The increase of transferred energies provides a qualitative new regime in electronuclear reactions. The possibility to suppress the long-range phenomena in these reactions opens a completely new window in the study of microscopic properties of nuclear matter at small distances.

The theoretical approach which allows us to do consistent calculation is based on the fact that in the high energy regime new small parameters (such as $\frac{q_{-}}{q_{+}}$) emerge. We demonstrate how the existence of these parameters connected to the reduction theorem which allows one to group potentially infinite number of rescattering amplitudes into a finite number covariant amplitudes.

Furthermore we identify the set of effective Feynman diagram rules which allows one to calculate these covariant amplitudes. This framework we call Generalized Eikonal Approximation (GEA). GEA is a covariant theory in which scattering amplitudes are represented as a sum of the diagrams corresponding to the $n$ 'th order rescattering of the knocked-out nucleon with residual nucleons in the target.

Applying these effective rules to the reaction under consideration we consequently calculate PWIA scattering amplitude, pair distortion amplitude, struck nucleon sin- 
gle rescattering amplitude and double rescattering amplitude. Calculations were carried out in details and final results are presented in a form suitable for programming and numerical calculations. Manifestly covariant nature of Feynman diagrams allowed us to preserve both the relativistic dynamics and kinematics of the scattering while identifying the low momentum nuclear part of the amplitude with nonrelativistic nuclear wave function. We have obtained the analytical expression for differential cross section of $\mathrm{e}+{ }^{3} \mathrm{He} \rightarrow \mathrm{e}^{\prime}+\mathrm{p}+\mathrm{p}+\mathrm{n}$ reaction. Numerical calculation of residual system's total and relative momentum distribution were performed which show reasonable agreement with available experimental data for $p n$ pairs. The $p p$ pairs situation looks less satisfactory. More data needed to draw a certain conclusion.

In this thesis, the theoretical framework of Generalized Eikonal Approximation, which was applied previously only for the case of two-body (deuteron) high energy break up reactions, has been practically implemented and shown to provide a valid description for more complex $A=3$ systems. 


\section{References}

[1] M. M. Sargsian et al., J. Phys. G 29, R1 (2003)

[2] M. M. Sargsian IJMP-E 10, 405 (2001)

[3] S. P. Merkuriev and L. D. Faddeev Quantum Scattering Theory for Systems of Several Particles (Dordrecht: Kluwer)

[4] R. P. Feynman, Photon Hadron Interactions, Benjamin Inc. 1972

[5] "Program Advisory Committee" at Jefferson Lab, Newport News, VA http://www.jlab.org/exp_prog/PACpage/index.html.

[6] L. L. Frankfurt, M. M. Sargsian and M. I. Strikman, Phys. Rev. C 56, 1124 (1997).

[7] R. J. Glauber Phys.Rev. 100, 242 (1955); Lectures in Theoretical Physics, v.1, ed. W. Brittain and L. G. Dunham, Interscience Publ., N.Y. 1959.

[8] K. I. Blomqvist et al., Phys. Lett. B 424, 33 (1998).

[9] H. Arenhovel, W. Leidemann and E. L. Tomusiak, Phys. Rev. C 52, 1232 (1995).

[10] M. Frodyma et al., Phys. Rev. C 47, 1599 (1993).

[11] L. L. Frankfurt, M. I. Strikman, D.B. Day and M. Sargsian, Phys. Rev. C 48, 2451 (1993).

[12] V. N. Gribov, JETP, 30, 709 (1970).

[13] L. Bertuchi, Il Nuovo Cimento A 11, 45 (1972).

[14] B. Blankleider and R. M. Woloshyn, Phys. Rev. C 29, 538 (1984).

[15] A. Nogga, H. Kamada and W. Glöckle, Nucl. Phys. A689, 357 (2001).

[16] R. A. Niyazov et al. [CLAS Collaboration], arXiv:nucl-ex/0308013.

[17] R. Niyazov, Ph. D. thesis, Old Dominion University (2003). 


\section{APPENDIX A. Three-nucleon bound systems}

The system which consists of three nucleons received special attention in the nuclear theory. First of all in this case we are dealing with the next simplest bound systems after deutron, namely ${ }^{3} \mathrm{H}$ and ${ }^{3} \mathrm{He}$ nuclei. Here is where the three-particle forces may exhibit themselves most clearly. It is therefore of evident importance to use what is known about two-nucleon forces in order to ascertain whether or not that information is adequate to account for the observed properties of $A=3$ system. Secondly, these systems can yield new data on the two-nucleon interactions itself. While considering scattering of two nucleons, third (spectator) particle may take up or impart enough energy to the two-nucleon subsystem to preserve the energy balance. So sum of initial energies of scatterers now may be different from the sum of their final energies. This off-energy-shell scatterings are important ingredients in the dynamics of nuclei other than deutron. In the two particle scatterings bound states cannot occur - we have as many scattering solutions as unperturbed solutions. However, if there are three or more particles participating in the process then it may well happen that two or more of them join up in the bound state. One of the most fundamental routes which was developed to handle three-particles problems in nonrelativistic quantum mechanics provided by method of Faddeév equations which we are going to discuss in this chapter.

Let us consider three interacting particles, labeled 1,2 and 3. Interaction between particles 1 and 2 we denote $V_{3}$, between 2 and $3-V_{1}$, and between 1 and 3 
$-V_{2}$. To facilitate derivation of Faddeév equations let us consider the specific case in which particle 2 scatters on particles 1 and 3. The Lippman-Schwinger equation for the transition operator $T$ is:

$$
T=V+V\left(E-H_{0}+i \varepsilon\right)^{-1} T .
$$

Interaction $V$ of incident particle 2 with the scatterers 1 and 3 is equal to

$$
V=V_{1}+V_{3}
$$

and the unperturbed Hamiltonian $H_{0}$ therefore can be expressed as

$$
H_{0}=V_{2}+K
$$

where $K$ is the total kinetic energy of the three particles

$$
K=\frac{\left|\mathbf{p}_{1}\right|^{2}}{2 m_{1}}+\frac{\left|\mathbf{p}_{2}\right|^{2}}{2 m_{2}}+\frac{\left|\mathbf{p}_{3}\right|^{2}}{2 m_{3}}
$$

The very small positive adiabatic switching factor $\varepsilon$ is introduced in order to avoid problems due to singularities which would otherwise be present when $E$ is equal to one of the egenvalues of $H_{0}$ and this is almost always the case for scattering problems.

Eq. (A.1) thus can be rewrited as

$$
T=\left(V_{1}+V_{3}\right)+\left(V_{1}+V_{3}\right) G_{2} T
$$

where

$$
G_{2} \equiv\left(E-K-V_{2}+i \varepsilon\right)^{-1} .
$$


Then

$$
\left\{1-\left(V_{1}+V_{3}\right) G_{2}\right\} T=\left(V_{1}+V_{3}\right)
$$

and

$$
T=\left\{1-\left(V_{1}+V_{3}\right) G_{2}\right\}^{-1}\left(V_{1}+V_{3}\right) .
$$

Let us now extract from $G_{2}$ the Green's function for the free particles $G$, defined as

$$
G \equiv(E-K+i \varepsilon)^{-1}
$$

With this definition we have

$$
\begin{aligned}
G_{2} & =\left[G^{-1}-V_{2}\right]^{-1}=\left[G^{-1}-V_{2}\right]^{-1} G^{-1} G= \\
& =\left[G^{-1}-V_{2}\right]^{-1}\left(G^{-1}-V_{2}+V_{2}\right) G=\left(1+\left[G^{-1}-V_{2}\right]^{-1} V_{2}\right) G= \\
& =\left(1+G_{2} V_{2}\right) G .
\end{aligned}
$$

Solving this for $G_{2}$ we get

$$
\begin{aligned}
G_{2}-G_{2} V_{2} G & =G_{2}\left[1-V_{2} G\right]=G, \\
G_{2} & =G\left[1-V_{2} G\right]^{-1} .
\end{aligned}
$$

In order to transform this further we first note that because $G\left[1-V_{2} G\right]=G-$ $G V_{2} G=\left[1-G V_{2}\right] G$ we can write the following identity

$$
G\left[1-V_{2} G\right]^{-1}=\left[1-G V_{2}\right]^{-1} G,
$$


so eq.(A.12) may be replaced with :

$$
G_{2}=\left[1-G V_{2}\right]^{-1} G \text {. }
$$

With this we now return to eq (A.8) and obtain a new form for transition operator $T:$

$$
\begin{aligned}
T & =\left\{1-\left(V_{1}+V_{3}\right)\left[G^{-1}-V_{2}\right]^{-1}\right\}^{-1}\left(V_{1}+V_{3}\right)= \\
& =\left\{1-\left(V_{1}+V_{3}\right)\left[1-G V_{2}\right]^{-1} G\right\}^{-1}\left(V_{1}+V_{3}\right)= \\
& =G^{-1}\left[1-G V_{2}-G\left(V_{1}+V_{3}\right)+G\left(V_{1}+V_{3}\right)\right] \times \\
& \times\left[1-G \sum_{k=1}^{3} V_{k}\right]^{-1} G\left(V_{1}+V_{3}\right),
\end{aligned}
$$

or

$$
T=\left(V_{1}+V_{3}\right)+\left(V_{1}+V_{3}\right)\left[1-G \sum_{k=1}^{3} V_{k}\right]^{-1} G\left(V_{1}+V_{3}\right)
$$

Let us define new quantities $T_{i j}$ by the means of equations

$$
T_{i j} \equiv V_{i} \delta_{i j}+V_{i}\left[1-G \sum_{l=1}^{3} V_{l}\right]^{-1} G V_{j}, \quad i, j=1,2,3 .
$$

Since we are considering particle 2 scatters on particles 1 and 3 we can represent the corresponding transition operator $T$ as a partial sum of $T_{i j}$ operators:

$$
T=\sum_{\substack{i \neq 2 \\ j \neq 2}} T_{i j}
$$

Our goal is to obtain the set of coupled equations for operators $T_{i j}$ and to express each one of them through others but not through itself. In order to do that we are 
going to transform eq.(A.17) by the following chain of identities:

$$
\begin{aligned}
T_{i j} & =V_{i} \delta_{i j}+V_{i}\left\{1-G \sum_{l=1}^{3} V_{l}+G \sum_{k=1}^{3} V_{k}\right\}\left[1-G \sum_{l=1}^{3} V_{l}\right]^{-1} G V_{j}= \\
& =V_{i} \delta_{i j}+V_{i} G V_{j}+V_{i} G\left(\sum_{k=1}^{3} V_{k}\right)\left[1-G \sum_{l=1}^{3} V_{l}\right]^{-1} G V_{j}= \\
& =V_{i} \delta_{i j}+V_{i} G \sum_{k=1}^{3}\left\{V_{k} \delta_{k j}+V_{k}\left[1-G \sum_{l=1}^{3} V_{l}\right]^{-1} G V_{j}\right\},
\end{aligned}
$$

but the quantity in braces is the same operator $T_{i j}$ we defined in eq.(A.17), therefore we arrive at

$$
T_{i j}=V_{i} \delta_{i j}+V_{i} G \sum_{k=1}^{3} T_{k j}
$$

We can solve eq.(A.20) for operator $T_{i j}$

$$
\begin{aligned}
\left(1-V_{i} G\right) T_{i j} & =V_{i} \delta_{i j}+V_{i} G \sum_{k \neq i} T_{k j} \\
T_{i j} & =\left(1-V_{i} G\right)^{-1} V_{i} \delta_{i j}+\left(1-V_{i} G\right)^{-1} V_{i} \sum_{k=1}^{3} \widetilde{G}_{i k} T_{k j}
\end{aligned}
$$

where we have introduced new operator $\widetilde{G}_{i k}$ :

$$
\widetilde{G}_{i k} \equiv G\left(1-\delta_{i k}\right)=(E-K+i \varepsilon)^{-1}\left(1-\delta_{i k}\right)
$$

Our final step is to make one more definition:

$$
\begin{aligned}
t_{i} & \equiv\left(1-V_{i} G\right)^{-1} V_{i}=V_{i}\left(1-G V_{i}\right)^{-1}= \\
& =V_{i}\left(G\left(G^{-1}-V_{i}\right)\right)^{-1}=V_{i}\left(G^{-1}-V_{i}\right)^{-1}\left(G^{-1}-V_{i}+V_{i}\right)= \\
& =V\left(1+\left(G-1-V_{i}\right)^{-1} V_{i}\right)=V_{i}+V_{i}\left(G^{-1}-V_{i}\right)^{-1} V_{i},
\end{aligned}
$$

or

$$
t_{i}=V_{i}+V_{i}\left(E-K-V_{i}+i \varepsilon\right)^{-1} V_{i} .
$$


One can show that so defined operator $t_{i}$ is the same transition operator $T$ given by eq.(A.1) for the case of two-particle scattering at energy $E$ by particles $j \neq i$ and $k \neq i$. Indeed, let us consider full Hamiltonian $H=H_{0}+V$, where unperturbed Hamiltonian is now just a kinetic energy $K$ of two interacting particles and $V$ is the coupling among them. Then eq.(A.1) might be transformed as

$$
\begin{aligned}
V^{-1} T & =1+\left(E-\left(H_{0}+V\right)+V+i \varepsilon\right)^{-1} T, \\
(E-H+V+i \varepsilon) V^{-1} T & =(E-H+V+i \varepsilon)+T \\
(E-H+i \varepsilon) V^{-1} T & =(E-H+i \varepsilon)+V, \\
T & =V+V(E-H+i \varepsilon)^{-1} V,
\end{aligned}
$$

which is identical to eq.(A.25). Returning to eq.(A.22) and substituting new-introduced operator $t_{i}$ we obtain one form of the famous Faddeév equations, namely

$$
T_{i j}=t_{i} \delta_{i j}+\sum_{k=1}^{3} t_{i} \widetilde{G}_{i k} T_{k j}, \quad i, j=1,2,3 .
$$

As one can clearly see, this set of coupled equations is completely inhomogeneous. In other words operator $T_{i j}$ does not appear on the right-hand $\operatorname{side}\left(\widetilde{G}_{i k}\right.$ gives 0 when $k=j$ ). Compared to initial Lippman-Schwinger equation eq.(A.1) this is significant improvement for three-particle problem. The kernels of corresponding integral equations can be shown to be compact for a broad range of potentials. The Faddeév equations are therefore an adequate starting point for a practicable solution to the problem of three interacting nucleons. 


\section{APPENDIX B. Scattering amplitude in the}

\section{momentum space}

Elastic scattering of two particles, just like any other two-body problem, can be treated as a scattering of one particle with the reduced mass in the field $U(\mathbf{r})$ of a fixed force center. Generally speaking, this field may have arbitrary spatial symmetry, the special case of central field we will denote as $U(r)$. Very far from interaction region the wave function of a scattered particle represents a mixture of a plane wave and a diverging spherical wave:

$$
\psi_{\mathbf{k}}^{(+)}(\mathbf{r}) \approx e^{i k r \mathbf{n} \mathbf{n}^{\prime}}+\frac{f\left(\mathbf{n}, \mathbf{n}^{\prime}\right)}{r} e^{i \mathbf{k r}}
$$

where scattering amplitude $f\left(\mathbf{n}, \mathbf{n}^{\prime}\right)$ depends on the directions of both incoming $(\mathbf{n})$ and outgoing $\left(\mathbf{n}^{\prime}\right)$ waves. Situation is much simpler in the case of centrally symmetric fields $U(r)$, where scattering amplitude only depends on the angle between $\mathbf{n}$ and $\mathbf{n}^{\prime}$. For those fields we can replace $f\left(\mathbf{n}, \mathbf{n}^{\prime}\right)=f\left(\mathbf{n} \cdot \mathbf{n}^{\prime}\right) \equiv f(\theta)$. Knowledge of $f(\theta)$ is sufficient for calculation of differential cross section:

$$
\frac{d \sigma}{d \Omega}=|f(\theta)|^{2}
$$

Solution of time independent Shrödinger equation, which describes scattering in the field $U(r)$, given by supperposition of continuum wave functions corresponding to motion of particle with known energy $E=k^{2} / 2 m$ and different values $l$ of orbital momenta:

$$
\psi_{\mathbf{k}}^{(+)}(\mathbf{r})=\frac{1}{2 k} \sum_{l=0}^{\infty} i^{l}(2 l+1) e^{i \delta_{l}} R_{k l}(r) P_{l}(\cos \theta)
$$


where $P_{l}$ are ordinary Legendre polynomials and $\delta_{l}$ are phase shifts of functions $R_{k l}(r)$. Latter are the solutions of the radial Shrödinger equation:

$$
\frac{1}{r^{2}} \frac{d}{d r}\left(r^{2} \frac{d R_{k l}}{d r}\right)+\left[k^{2}-\frac{l(l+1)}{r^{2}}-2 m U(r)\right] R_{k l}=0
$$

Using asymptotical form of $R_{k l}(r)$ one can express scattering amplitude through the phase shifts $\delta_{l}$

$$
f^{\text {on shell }}(\theta)=\frac{1}{2 i k} \sum_{l=0}^{\infty}(2 l+1)\left(\exp \left(2 i \delta_{l}\right)-1\right) P_{l}(\cos \theta)
$$

The "on shell" tag should remind that this expansion is valid only for scattering of unbound (on-mass-shell) particles, when the corresponding field $U(r)$ is assumed to be centrally symmetric. One can't use eq.(B.5) for bound (off shell) scatterers. Instead of central fields let us consider arbitrary $U(\mathbf{r})$ and deduce a general expression which connects the scattering amplitude $f\left(\mathbf{n}, \mathbf{n}^{\prime}\right)$ with the momentum space wave functions $\Psi_{\mathbf{k}}^{(+)}(\mathbf{q})$. The coordinate space Fourier-components of these functions in asymptotic limit are given by eq.(B.1).

As usually, we define direct and inverse Fourier transformations as

$$
\Psi_{\mathbf{k}}^{(+)}(\mathbf{q})=\int \psi_{\mathbf{k}}^{(+)}(\mathbf{r}) e^{-i \mathbf{q r}} d V
$$

and

$$
\psi_{\mathbf{k}}^{(+)}(\mathbf{r})=\int \Psi_{\mathbf{k}}^{(+)}(\mathbf{q}) e^{i \mathbf{q} \mathbf{r}} \frac{d^{3} q}{(2 \pi)^{3}}
$$

Time independent Shrödinger equation in the coordinate representation given by

$$
-\frac{1}{2 m} \Delta \psi_{\mathbf{k}}^{(+)}(\mathbf{r})+[U(\mathbf{r})-E] \psi_{\mathbf{k}}^{(+)}(\mathbf{r})=0
$$


In the momentum space one rewrites this equation as

$$
\left(\frac{q^{2}}{2 m}-E\right) \Psi_{\mathbf{k}}^{(+)}(\mathbf{q})+\int U\left(\mathbf{q}-\mathbf{q}^{\prime}\right) \Psi_{\mathbf{k}}^{(+)}\left(\mathbf{q}^{\prime}\right) \frac{d^{3} q^{\prime}}{(2 \pi)^{3}}=0
$$

where $U(\mathbf{q})$ is a Fourier-component of $U(\mathbf{r})$ :

$$
U(\mathbf{q})=\int U(\mathbf{r}) e^{-i \mathbf{q r}} d V
$$

Replacing last term in eq.(B.1) by function $\chi_{\mathbf{k}}(\mathbf{r})$, which is a diverging spherical wave as $r \rightarrow \infty$, one has

$$
\psi_{\mathbf{k}}^{(+)}(\mathbf{r})=e^{i \mathbf{k r}}+\chi_{\mathbf{k}}(\mathbf{r})
$$

The corresponding Fourier-image of this equation has the form of

$$
\Psi_{\mathbf{k}}^{(+)}(\mathbf{q})=(2 \pi)^{3} \delta^{3}(\mathbf{q}-\mathbf{k})+\chi_{\mathbf{k}}(\mathbf{q})
$$

Plugging this in eq.(B.9) and replacing $E=k^{2} / 2 m$ we get

$$
\begin{gathered}
\frac{(2 \pi)^{3}}{2 m}\left(q^{2}-k^{2}\right) \delta^{3}(\mathbf{q}-\mathbf{k})+\frac{1}{2 m}\left(q^{2}-k^{2}\right) \chi_{\mathbf{k}}(\mathbf{q})= \\
=\int U\left(\mathbf{q}-\mathbf{q}^{\prime}\right)(2 \pi)^{3} \delta^{3}(\mathbf{q}-\mathbf{k}) \frac{d^{3} q^{\prime}}{(2 \pi)^{3}}+\int U\left(\mathbf{q}-\mathbf{q}^{\prime}\right) \chi_{\mathbf{k}}\left(\mathbf{q}^{\prime}\right) \frac{d^{3} q^{\prime}}{(2 \pi)^{3}} .
\end{gathered}
$$

First term on the left hand side identically vanishes and we arrive at

$$
\frac{1}{2 m}\left(k^{2}-q^{2}\right) \chi_{\mathbf{k}}(\mathbf{q})=U(\mathbf{q}-\mathbf{k})+\int U\left(\mathbf{q}-\mathbf{q}^{\prime}\right) \chi_{\mathbf{k}}\left(\mathbf{q}^{\prime}\right) \frac{d^{3} q^{\prime}}{(2 \pi)^{3}}
$$

Defining a new function $F(\mathbf{k}, \mathbf{q})$ as

$$
\chi_{\mathbf{k}}(\mathbf{q})=2 m \frac{F(\mathbf{k}, \mathbf{q})}{q^{2}-k^{2}-i \varepsilon}
$$


we immediately get from eq.(B.14)

$$
F(\mathbf{k}, \mathbf{q})=-U(\mathbf{q}-\mathbf{k})-2 m \int \frac{U\left(\mathbf{q}-\mathbf{q}^{\prime}\right) F\left(\mathbf{k}, \mathbf{q}^{\prime}\right)}{q^{2}-k^{2}-i \varepsilon} \frac{d^{3} q^{\prime}}{(2 \pi)^{3}} .
$$

Infinitesimally small term $i \varepsilon$, which was added to avoid singularities at $q= \pm k$ could be chosen to carry either "+" or "-" sign. Depending on that sign the integral in eq.(B.16) may have two different values. Indeed, according to contour integration theorem, if $z=\infty$ is at least second order 0 for analitical function $f(z)$, then

$$
\int_{-\infty}^{\infty} f(x) d x=2 \pi i \sum_{\nu=1}^{n} \operatorname{Res}_{a_{\nu}} f(z), \quad a_{\nu} \in+\operatorname{Im} z .
$$

As we write

$$
\begin{aligned}
& q^{2}-k^{2}+i \varepsilon=(q-k+i \varepsilon)(q+k-i \varepsilon), \\
& q^{2}-k^{2}-i \varepsilon=(q-k-i \varepsilon)(q+k+i \varepsilon),
\end{aligned}
$$

and check with the Fig. 17 we see that $+i \varepsilon$ determines the value of integral through the residue in $-k$ whereas $-i \varepsilon$ idicates that residue should be taken at $+k$. Choice

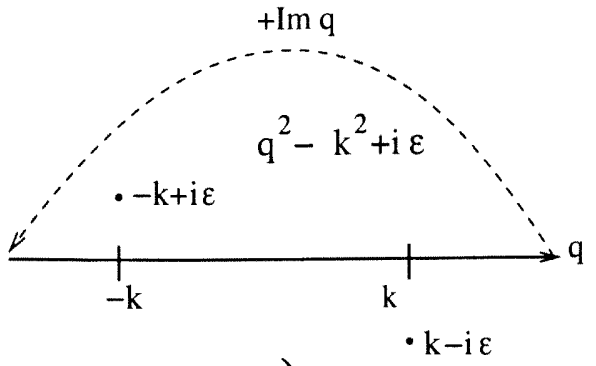

a)

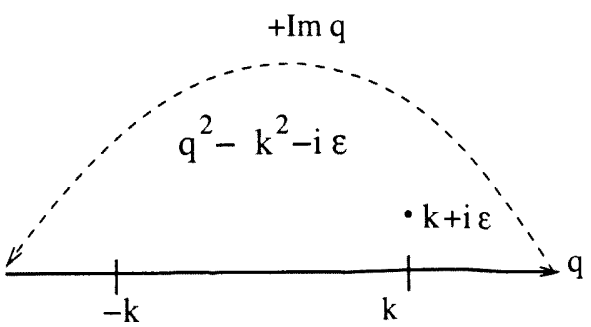

$\cdot-\mathrm{k}-\mathrm{i} \varepsilon$

b)

Figure 17: a) Poles corresponding to the choice $+i \varepsilon$, b) same for $-i \varepsilon$.

b) automatically insures that inverse Fourier transformations of $\chi_{\mathbf{k}}(\mathbf{q})$ will return 
correct diverging behavior of spherical wave $\chi_{\mathbf{k}}(\mathbf{r})$ from eq.(B.11). To prove it we apply transformation of eq.(B.7) to eq.(B.15). With the definition $\mathbf{n}^{\prime}=\mathbf{r} / r$ we have

$$
\chi_{\mathbf{k}}\left(r \mathbf{n}^{\prime}\right)=2 m \int \frac{F(\mathbf{k}, \mathbf{q})}{q^{2}-k^{2}-i \varepsilon} e^{i q r \cos \theta} \frac{q^{2} d q d \phi d(\cos \theta)}{(2 \pi)^{3}}
$$

where we have aligned $z$-direction with $\mathbf{n}^{\prime}$.

Integration over $d \phi$ gives $2 \pi$, whereas integral over $d \mu \equiv d(\cos \theta)$ can be worked out if we slightly bend the path of integration toward the upper-half complex plane (Fig. 18) keeping boundaries fixed at -1 and 1. Since $r$ is arbitrary large exponent

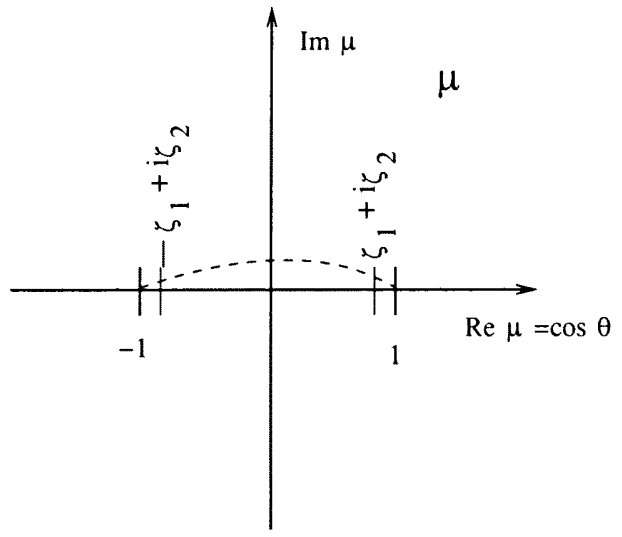

Figure 18: Integral over $d \mu=d(\cos \theta)$.

$e^{i q r \mu}$ is highly oscillating function and the value of integral mainly defined by the regions where $\mathbf{q} / q= \pm \mathbf{n}^{\prime}$. In that regions $F(\mathbf{k}, \mathbf{q}) \approx F\left(\mathbf{k}, \pm q \mathbf{n}^{\prime}\right)$ and can be pulled out in front of the integral. Observing that $e^{i q r \mu}$ damping very fast as it departs from the boundaries \pm 1 we arrive at

$$
\chi_{\mathbf{k}}\left(r \mathbf{n}^{\prime}\right)=-2 m \frac{2 \pi i}{r}\left(\int_{0}^{\infty} \frac{F\left(\mathbf{k}, q \mathbf{n}^{\prime}\right) e^{i q r}}{q^{2}-k^{2}-i \varepsilon} \frac{q d q}{(2 \pi)^{3}}-\int_{0}^{\infty} \frac{F\left(\mathbf{k},-q \mathbf{n}^{\prime}\right) e^{-i q r}}{q^{2}-k^{2}-i \varepsilon} \frac{q d q}{(2 \pi)^{3}}\right) .
$$


Substitution $q \rightarrow-q$ in the last integral yields

$$
\chi_{\mathbf{k}}\left(r \mathbf{n}^{\prime}\right)=-\frac{i m}{2 \pi^{2} r} \int_{-\infty}^{\infty} \frac{F\left(\mathbf{k}, q \mathbf{n}^{\prime}\right) e^{i q r} q d q}{q^{2}-k^{2}-i \varepsilon}
$$

Using contour integration theorem (see eq.(B.17)) and setting $\mathbf{n} \equiv \mathbf{k} / k$ one obtains

$$
\chi_{\mathbf{k}}(\mathbf{r})=\frac{m}{2 \pi} \frac{e^{i k r}}{r} F\left(k \mathbf{n}, k \mathbf{n}^{\prime}\right)
$$

which is precisely what one would expect to get for diverging spherical wave.

From eq.(B.1) we identify

$$
f\left(\mathbf{n}, \mathbf{n}^{\prime}\right)=\frac{m}{2 \pi} F\left(k \mathbf{n}, k \mathbf{n}^{\prime}\right)
$$

and eq.(B.16) can be rewritten as

$$
\chi_{\mathbf{k}}(\mathbf{q})=4 \pi \frac{f\left(\mathbf{n}, \mathbf{n}^{\prime}\right)}{q^{2}-k^{2}-i \varepsilon} .
$$

With this we are able to write down the desired expression for $\Psi_{\mathbf{k}}^{(+)}(\mathbf{q})$ :

$$
\Psi_{\mathbf{k}}^{(+)}(\mathbf{q})=(2 \pi)^{3} \delta^{3}(\mathbf{q}-\mathbf{k})+4 \pi \frac{f\left(\mathbf{n}, \mathbf{n}^{\prime}\right)}{q^{2}-k^{2}-i \varepsilon}
$$




\section{APPENDIX C. Structure functions in differential}

\section{crossections}

We are considering exclusive $\left(e+A \rightarrow e^{\prime}+p_{f}+A-1\right)$ reaction, where insident electron with 4-vector $k_{1}=\left(E_{1}, \mathbf{k}_{1}\right)$ knocks out nuclon from target nucleus $\mathrm{A}$, which 4-vector is $p_{A}=\left(E_{A}, \mathbf{p}_{A}\right)$. It is convinient to work in the frame where target is stationar, so $\mathbf{p}_{A}=0$ and $p_{A}=M_{A}$. Exclusive nature of reaction allows to detect scattered electron energy-momentum $k_{2}=\left(E_{2}, \mathbf{k}_{2}\right)$, as well as the one for knocked out nuclon $p_{f}=\left(E_{f}, \mathbf{p}_{f}\right)$. The differense between initial and final electron momenta is the 4-momentum transfered to the $\mathrm{A}, q=\left(q_{0}, \mathbf{q}\right)$. The most general considerations allow us to represent differential cross section for this reaction as

$$
d \sigma=\frac{2 \alpha^{2} E_{2}}{E_{1} Q^{4}}|\mathcal{M}|^{2} d E_{2} d \Omega_{2} d^{3} \mathbf{p}_{f}
$$

where $\alpha$ is the fine structure constant, $Q^{2}=-q^{2}$ and $|\mathcal{M}|^{2}$ is a Lorentz invariant quantity representing scalar contraction of leptonic and hadronic tensors

$$
|\mathcal{M}|^{2}=l_{\mu \nu} w^{\mu \nu}
$$

After averaging over initial and summing over final states QED yelds for leptonic tensor to

$$
l^{\mu \nu}=2\left(k_{2}^{\mu} k_{1}^{\nu}+k_{2}^{\nu} k_{1}^{\mu}-g^{\mu \nu} k_{1} \cdot k_{2}\right)
$$

Unlike QED, the theory of strong interactions(QCD) is not complete yet, so purely analitical expression for hadronic tensor does not exist. However fairly simple 
formula for that quantity can be obtained with the help of very general physical assumptions about the nature of hadronic current. Namely, we can expect this current to be gauge and Lorentz invariant. We also can assume that $w^{\mu \nu}$ does not change as we swap it's superscipts $\mu \leftrightarrow \nu$. Thus, we can write

$$
\begin{gathered}
w^{\mu \nu}=T_{0}\left(g_{\mu \nu}-\frac{q_{\mu} q_{\nu}}{q^{2}}\right)+ \\
+\frac{T_{1}}{M_{A}^{2}}\left(p_{A}^{\mu}-\frac{p_{A} \cdot q}{q^{2}} q^{\mu}\right)\left(p_{A}^{\nu}-\frac{p_{A} \cdot q}{q^{2}} q^{\nu}\right)+\frac{T_{2}}{M_{f}^{2}}\left(p_{f}^{\mu}-\frac{p_{f} \cdot q}{q^{2}} q^{\mu}\right)\left(p_{f}^{\nu}-\frac{p_{f} \cdot q}{q^{2}} q^{\nu}\right)+ \\
+\frac{T_{3}}{M_{A} M_{f}}\left(\left(p_{A}^{\mu}-\frac{p_{A} \cdot q}{q^{2}} q^{\mu}\right)\left(p_{f}^{\nu}-\frac{p_{f} \cdot q}{q^{2}} q^{\nu}\right)+\left(p_{f}^{\mu}-\frac{p_{f} \cdot q}{q^{2}} q^{\mu}\right)\left(p_{A}^{\nu}-\frac{p_{A} \cdot q}{q^{2}} q^{\nu}\right)\right)
\end{gathered}
$$

Scalar factors $T_{0}, T_{1}, T_{2}$ and $T_{3}$ contains all information about nuclon's nonelimentar structure and can be parametrised from experimental data. More subjectively, these factors can be extracted from four independent structure(or responce) functions which are directly measured in experiment. Let us show that one can actually break down right hand side of eq.(C.2) in terms of only 4 independent mesurable functions and then exract $T$-factors from them.

First note, that formally instead of claimed 4 terms contraction eq.(C.2) involves 16. However $\mu \leftrightarrow \nu$ symmetry reduces right away that number to 10 :

$$
\begin{aligned}
|\mathcal{M}|^{2}= & l_{0 \nu} w^{0 \nu}-l_{1 \nu} w^{1 \nu}-l_{2 \nu} w^{2 \nu}-l_{3 \nu} w^{3 \nu}= \\
= & l_{00} w^{00}-2 l_{01} w^{01}-2 l_{02} w^{02}-2 l_{03} w^{03}+ \\
& +2 l_{12} w^{12}+2 l_{13} w^{13}+2 l_{23} w^{23}+ \\
& +l_{11} w^{11}+l_{22} w^{22}+l_{33} w^{33}
\end{aligned}
$$


One can simplify this expression further choosing De Forest's coordinate system. We free to align $\hat{z}$ axis with $\mathbf{q}$, so $q=\left(q_{0}, 0,0, q_{z}\right)$. It is also convinient to define $(y z)$ coordinate plane as $\left(p_{f} q\right)$ plane (which is the reaction plane), so $p_{f_{x}}=0$. As noted above we are considering stationar target nucleus, $\mathbf{p}_{A}=0$. Checking with eq.(C.4) it is easy to see that except $w^{11}$ term all other hadronic tensor elements with " 1 " superscript identically equal to zero. Thus we can rewrite eq.(C.5) without $w^{01}, w^{12}$ and $w^{13}$ terms:

$$
|\mathcal{M}|^{2}=l_{00} w^{00}-2 l_{02} w^{02}-2 l_{0 q} w^{0 q}+2 l_{2 q} w^{2 q}+l_{11} w^{11}+l_{22} w^{22}+l_{q q} w^{q q} .(\mathrm{C} .6
$$

It is time now to use continuity equation for electron current providing us with

$$
q^{\mu} l_{\mu \nu}=0
$$

or, since $\hat{z} \| \mathbf{q}$,

$$
q_{0} l_{0 \nu}-q_{\mathrm{v}} l_{q \nu}=0
$$

where $q_{\mathrm{v}} \equiv|\mathbf{q}|=q_{z}$. Using this we can express

$$
l_{q \nu}=\left(\frac{q_{0}}{q_{\mathrm{v}}}\right) l_{0 \nu}, \quad l_{q q}=\left(\frac{q_{0}}{q_{\mathrm{v}}}\right)^{2} l_{00} .
$$

Plugging eq.(C.9) in eq.(C.6) and collecting terms we obtain

$$
\begin{aligned}
|\mathcal{M}|^{2}= & l_{00} w^{00}-2 l_{02} w^{02}-2\left(\frac{q_{0}}{q_{\mathrm{v}}}\right) l_{00} w^{0 q}+ \\
& +2\left(\frac{q_{0}}{q_{\mathrm{v}}}\right) l_{20} w^{2 q}+l_{11} w^{11}+l_{22} w^{22}+\left(\frac{q_{0}}{q_{\mathrm{v}}}\right)^{2} l_{00} w^{q q}= \\
= & l_{00}\left[w^{00}-2\left(\frac{q_{0}}{q_{\mathrm{v}}}\right) w^{0 q}+\left(\frac{q_{0}}{q_{\mathrm{v}}}\right)^{2} w^{q q}\right]+ \\
& +2 l_{20}\left[\left(\frac{q_{0}}{q_{\mathrm{v}}}\right) w^{2 q}-w^{02}\right]+l_{11} w^{11}+l_{22} w^{22} .
\end{aligned}
$$


Let us rewrite two last terms of eq.(C.10) in slightly different fashion:

$$
\begin{aligned}
l_{11} w^{11}+l_{22} w^{22} & =\left(\frac{l_{22}+l_{11}}{2}+\frac{l_{22}-l_{11}}{2}\right) w^{22}+\left(\frac{l_{22}+l_{11}}{2}-\frac{l_{22}-l_{11}}{2}\right) w^{11}= \\
& \left.=\left(\frac{l_{22}-l_{11}}{2}\right)\left[w^{22}-w^{11}\right]+\left(\frac{l_{22}+l_{11}}{2}\right)\left[w^{22}+w^{11}\right], \quad \text { (C. } 11\right)
\end{aligned}
$$

so eq.(C.10) can be expressed as

$$
\begin{aligned}
|\mathcal{M}|^{2}= & l_{00}\left[w^{00}-2\left(\frac{q_{0}}{q_{\mathrm{v}}}\right) w^{0 q}+\left(\frac{q_{0}}{q_{\mathrm{v}}}\right)^{2} w^{q q}\right]+ \\
& +2 l_{20}\left[\left(\frac{q_{0}}{q_{\mathrm{v}}}\right) w^{2 q}-w^{02}\right]+ \\
& +\left(\frac{l_{22}-l_{11}}{2}\right)\left[w^{22}-w^{11}\right]+\left(\frac{l_{22}+l_{11}}{2}\right)\left[w^{22}+w^{11}\right] .
\end{aligned}
$$

Now we are ready to define 4 structure functions:

$$
\begin{aligned}
W_{L} & =\left(\frac{q_{\mathrm{v}}}{Q}\right)^{4}\left[w^{00}-2\left(\frac{q_{0}}{q_{\mathrm{v}}}\right) w^{0 q}+\left(\frac{q_{0}}{q_{\mathrm{v}}}\right)^{2} w^{q q}\right] \\
W_{T L} & =-2\left(\frac{q_{\mathrm{v}}}{Q}\right)^{2}\left[\left(\frac{q_{0}}{q_{\mathrm{v}}}\right) w^{2 q}-w^{02}\right] \\
W_{T T} & =\left[w^{22}-w^{11}\right] \\
W_{T} & =\left[w^{22}+w^{11}\right]
\end{aligned}
$$

which are making eq.(C.12) to look like

$$
|\mathcal{M}|^{2}=l_{00}\left(\frac{Q}{q_{\mathrm{v}}}\right)^{4} W_{L}-l_{20}\left(\frac{Q}{q_{\mathrm{v}}}\right)^{2} W_{T L}+\left(\frac{l_{22}-l_{11}}{2}\right) W_{T T}+\left(\frac{l_{22}+l_{11}}{2}\right) W_{T} \text { (C.17) }
$$

Substituting well-known expressions for componets of leptonic tensor into eq.(C.17) we will obtain final formula for $|\mathcal{M}|^{2}$ :

$$
\begin{aligned}
|\mathcal{M}|^{2}= & 2 E_{1} E_{2} \cos ^{2} \frac{\theta}{2}\left[\frac{Q^{4}}{q_{\mathrm{v}}^{4}} W_{L}+\left(\frac{Q^{2}}{2 \mathrm{v}^{2}}+\tan ^{2} \frac{\theta}{2}\right) W_{T}+\right. \\
& \left.+\frac{Q^{2}}{q_{\mathrm{v}}^{2}}\left(\frac{Q^{2}}{q_{\mathrm{v}}^{2}}+\tan ^{2} \frac{\theta}{2}\right)^{\frac{1}{2}} W_{T L} \cos \phi+\frac{Q^{2}}{2 q_{\mathrm{v}}^{2}} W_{T T} \cos 2 \phi\right] .
\end{aligned}
$$


When plugging this into eq.(C.1) we obtain well-known differential cross section formula for exclusive $\left(e+A \rightarrow e^{\prime}+p_{f}+A-1\right)$ reactions:

$$
\begin{aligned}
\frac{d \sigma}{d E_{2} d \Omega_{2} d^{3} \mathbf{p}_{f}}= & \sigma_{\mathrm{Mott}}\left[\frac{Q^{4}}{q_{\mathrm{v}}^{4}} W_{L}+\left(\frac{Q^{2}}{2 q_{\mathrm{v}}^{2}}+\tan ^{2} \frac{\theta}{2}\right) W_{T}+\right. \\
& \left.+\frac{Q^{2}}{q_{\mathrm{v}}^{2}}\left(\frac{Q^{2}}{q_{\mathrm{v}}^{2}}+\tan ^{2} \frac{\theta}{2}\right)^{\frac{1}{2}} W_{T L} \cos \phi+\frac{Q^{2}}{2 q_{\mathrm{v}}^{2}} W_{T T} \cos 2 \phi\right]
\end{aligned}
$$

where $\sigma_{\text {Mott }}=\alpha^{2} \cos ^{2} \frac{\theta}{2} / 4 E_{1}^{2} \sin ^{4} \frac{\theta}{2}$.

Just as it was with leptonic current we can expect hadronic current to be gauge invariant. Using this one can clarify the physical meaning of structure functions defined in eqs.(C.13)-(C.16) by expressing hadronic tensor elements via each other, exactly like it was done in eq.(C.9). This will establish the following relations between structure functions from the one hand and hadronic current $J$ and charge density $\rho$ from the other:

$$
\begin{aligned}
W_{L} & =w^{00}=\left\langle\rho \rho^{+}\right\rangle, \\
W_{T L} \cos \phi & =-2 w^{02}=-\left\langle\rho J_{2}^{+}+J_{2} \rho^{+}\right\rangle, \\
W_{T T} \cos 2 \phi & =w^{22}-w^{11}=\left\langle J_{2} J_{2}^{+}-J_{1} J_{1}^{+}\right\rangle, \\
W_{T} & =w^{22}+w^{11}=\left\langle J_{2} J_{2}^{+}+J_{1} J_{1}^{+}\right\rangle,
\end{aligned}
$$

with angular brackets denoting average over initial states and summation over final states.

These representations show explicitly that, indeed, structure functions are measurable quantities. In some sence they provide a direct bridge between theoretical and experimental frameworks. To show this it is enough to substitute $w^{\mu \nu}$ tensor 
elements in last equations with their values from eq.(C.4). Doing that we get

$$
\begin{aligned}
W_{L} & =\frac{T_{0} q_{\mathrm{v}}^{2}}{Q^{2}}+\frac{T_{1} q_{\mathrm{v}}^{4}}{Q^{4}}+\frac{T_{2} q_{\mathrm{v}}^{2}}{M_{f}^{2} Q^{4}}\left(q_{\mathrm{v}} E_{f}-p_{f_{z}} q_{0}\right)^{2}+\frac{2 T_{3} q_{\mathrm{v}}^{3}}{M_{f} Q^{4}}\left(q_{\mathrm{v}} E_{f}-p_{f_{z}} q_{0}\right)(\mathrm{C} 2) \\
W_{T L} & =-2 T_{2} \frac{p_{f_{y}} q_{\mathrm{v}}}{M_{f}^{2} Q^{2}}\left(q_{\mathrm{v}} E_{f}-p_{f_{z}} q_{0}\right)-2 T_{3} \frac{p_{f_{y}} q_{\mathrm{v}}^{2}}{M_{f} Q^{2}} \\
W_{T T} & =T_{2} \frac{p_{f_{y}}{ }^{2}}{M_{f}^{2}} \\
W_{T} & =-2 T_{0}+T_{2} \frac{p_{f_{y}}{ }^{2}}{M_{f}^{2}}
\end{aligned}
$$

For given reaction kinematics one can actually mesuare structure functions, then solve Eqs. eqs.(C.24)-(C.27) for $T_{0}, T_{1}, T_{2}$ and $T_{3}$ and obtain desired parametrisations for those quantities. 


\section{APPENDIX D. NN diffractive parameters}

function sigma_pp_tot(s)

C pp total cross section fit in [mb] (sigma_pp)

common/par/pi,pm,pmp,pmn,tm,eb ! pm $\rightarrow$ mass of the proton dimension $B(10)$

$\mathrm{ep}=(\mathrm{s}-2.0 * \mathrm{pm} * * 2) /(2.0 * \mathrm{pm})$

$\mathrm{p}=\operatorname{sqrt}(\mathrm{ep} * * 2-\mathrm{pm} * * 2)$

$\mathrm{pcm}=\mathrm{pm} * \mathrm{p} / \mathrm{sqrt}(\mathrm{s})$

$\mathrm{pcm}=\mathrm{pcm} / \mathrm{sqrt}(0.389385)$

! in $\mathrm{GeV}$

if (p.1t.3.68) then

! in $1 / \operatorname{sqrt}(\mathrm{mb})$

call Bystricky_dat $(p, 0.0,0, B)$ ! using SAID's analysis

stot $=4 . * \mathrm{pi} / \mathrm{pcm} *(\mathrm{~B}(6)+\mathrm{B}(7)) / 2$.

elseif ( p.le.5.87) then

$\mathrm{x} 1=3.68$

$\mathrm{y} 1=41.39135$

$\mathrm{x} 2=3.9$

$\mathrm{y} 2=41.19135$

$\mathrm{x} 3=5.870000$

$\mathrm{y} 3=40.42657$

stot $=$ cubic_spline $(p, x 1, x 2, x 3, y 1, y 2, y 3)$

elseif ( p.le.12.) then

$\mathrm{x} 1=5.87$

$\mathrm{y} 1=40.42657$

$\mathrm{x} 2=9$.

$\mathrm{y} 2=40.19135$

$\mathrm{x} 3=12.00000$

$\mathrm{y} 3=40.01630$

stot $=$ cubic_spline $(\mathrm{p}, \mathrm{x} 1, \mathrm{x} 2, \mathrm{x} 3, \mathrm{y} 1, \mathrm{y} 2, \mathrm{y} 3)$

else

stot $=48.0+0.522 * a \log (p) * * 2-4.51 * a \log (p)$

endif

sigma_pp_tot $=$ stot

return

end 
function sigma_pn_tot(s)

C

pn total cross section fit in [mb] (sigma_pn)

common/par/pi,pm,pmp,pmn,tm, eb ! pm $\rightarrow$ mass of the proton dimension $\mathrm{B}(10)$

$\mathrm{ep}=(\mathrm{s}-2.0 * \mathrm{pm} * * 2) /(2.0 * \mathrm{pm})$

$\mathrm{p}=\operatorname{sqrt}(\mathrm{ep} * * 2-\mathrm{pm} * * 2)$

$\mathrm{pcm}=\mathrm{pm} * \mathrm{p} / \mathrm{sqrt}(\mathrm{s})$

! in $\mathrm{GeV}$

$\mathrm{pcm}=\mathrm{pcm} / \mathrm{sqrt}(0.389385)$

! in $1 /$ sqrt (mb)

if (p.1t.1.4) then

call Bystricky_dat $(p, 0.0,1, B)$ ! using SAID's analysis

stot $=4 . * \mathrm{pi} / \mathrm{pcm} *(\mathrm{~B}(6)+\mathrm{B}(7)) / 2$.

elseif(p.le.3.1) then

$\mathrm{x} 1=1.4$

$\mathrm{y} 1=38.42728$

$\mathrm{x} 2=2.07800$

$\mathrm{y} 2=41.905$

$\mathrm{x} 3=3.1$

$\mathrm{y} 3=43.13159$

stot $=$ cubic_spline $(p, x 1, x 2, x 3, y 1, y 2, y 3)$

else

stot $=47.3+0.513 * \operatorname{alog}(p) * * 2-4.27 * \operatorname{alog}(p)$

endif

sigma_pn_tot $=$ stot

return

end

function a_pp(s)

C $\mathrm{pp} R e / \mathrm{Im}$ amplidtudes ratio parameter (alpha_pp)

common/par/pi,pm,pmp,pmn,tm,eb ! pm $\rightarrow$ mass of the proton dimension $\mathrm{B}(10)$

$\mathrm{ep}=(\mathrm{s}-2.0 * \mathrm{pm} * * 2) /(2.0 * \mathrm{pm})$

$\mathrm{p}=\operatorname{sqrt}(\mathrm{ep} * * 2-\mathrm{pm} * * 2)$

$\mathrm{a}_{-} \mathrm{pp}=0.0$

if (p.1t.3.68) then

call Bystricky_dat $(p, 0.0,0, B)$ ! using SAID's analysis

$\mathrm{a}_{-} \mathrm{pp}=(\mathrm{B}(1)+\mathrm{B}(2)) /(\mathrm{B}(6)+\mathrm{B}(7))$

return 


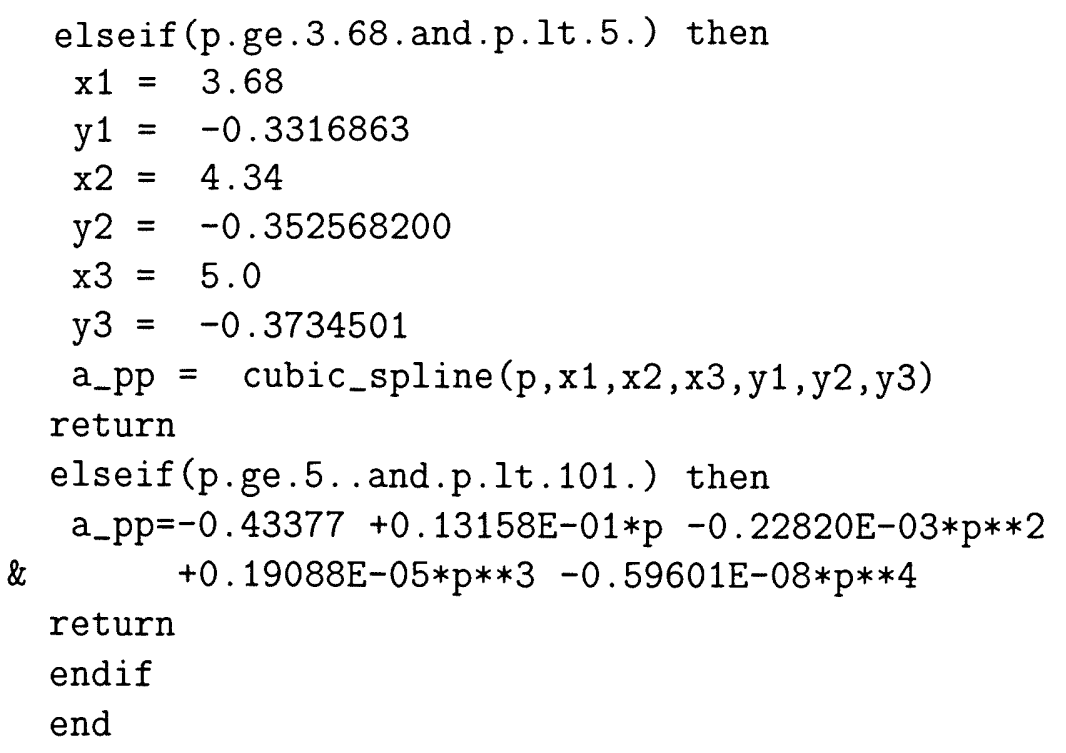

function a_pn(s)

C pn Re/Im amplidtudes ratio parameter (alpha_pn)

common/par/pi,pm,pmp,pmn,tm,eb ! pm $\rightarrow$ mass of the proton dimension $B(10)$

$\mathrm{ep}=(\mathrm{s}-2.0 * \mathrm{pm} * * 2) /(2.0 * \mathrm{pm})$

$\mathrm{p}=\operatorname{sqrt}(\mathrm{ep} * * 2-\mathrm{pm} * * 2)$

$a_{-} p n=0.0$

if (p.1t.1.4) then

call Bystricky_dat $(p, 0.0,1, B)$ ! using SAID's analysis

$\mathrm{a}_{-} \mathrm{pn}=(\mathrm{B}(1)+\mathrm{B}(2)) /(\mathrm{B}(6)+\mathrm{B}(7))$

return

elseif(p.ge.1.4.and.p.1t.3.) then

$$
\begin{aligned}
& \mathrm{x} 1=1.4000 \\
& \mathrm{y} 1=-0.2845016 \\
& \mathrm{x} 2=1.9 \\
& \mathrm{y} 2=-0.43915290 \\
& \mathrm{x} 3=3.0 \\
& \mathrm{y} 3=-0.4938042 \\
& \text { a_pn }=\text { cubic_spline }(p, x 1, \mathrm{x} 2, \mathrm{x} 3, \mathrm{y} 1, \mathrm{y} 2, \mathrm{y} 3)
\end{aligned}
$$

return

elseif (p.ge.3..and.p.1t.101.) then

$a_{-} \mathrm{pn}=-0.56207+0.24223 \mathrm{E}-01 * \mathrm{p}-0.50362 \mathrm{E}-03 * \mathrm{p} * * 2$

$\&$

$+0.48408 \mathrm{E}-05 * \mathrm{p} * * 3-0.17331 \mathrm{E}-07 * \mathrm{p} * * 4$ 
endif

return

end

function $b p(s)$

C pp Slope parameter (Bpp)

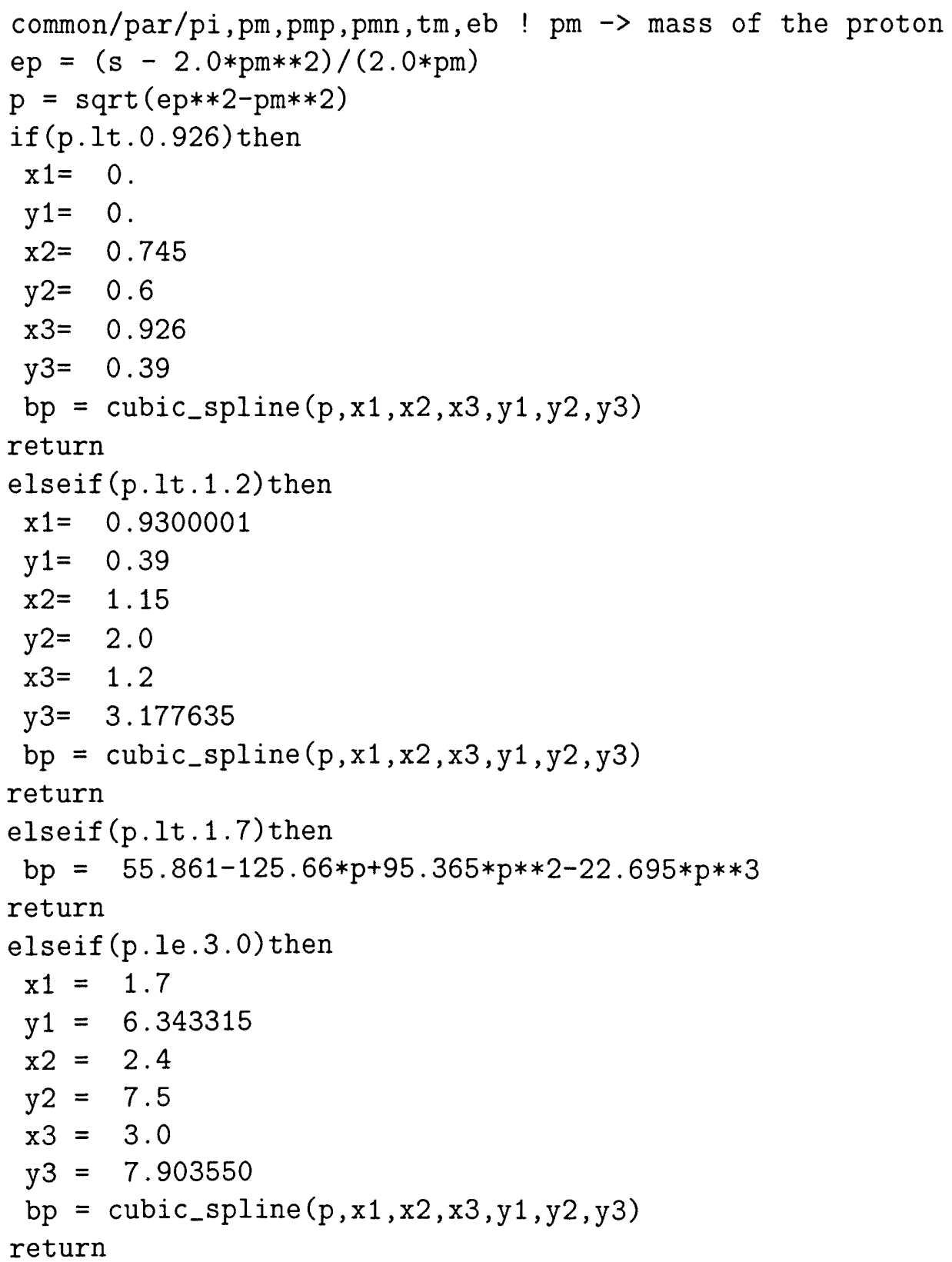
return 
else

$\mathrm{bp}=8.22+1.10 * \log (\mathrm{p} / 4$.

endif

return

end

function bn(s)

C pn Slope parameter (Bpn)

common/par/pi,pm,pmp,pmn,tm,eb ! pm $\rightarrow$ mass of the proton dimension B1(10), B2(10)

ep $=(\mathrm{s}-2.0 * \mathrm{pm} * * 2) /(2.0 * \mathrm{pm})$

$\mathrm{p}=\operatorname{sqrt}(\mathrm{ep} * * 2-\mathrm{pm} * * 2)$

if (p.1t.0.8) then

$\mathrm{x} 1=0$.

$\mathrm{y} 1=0$.

$\mathrm{x} 2=0.545$

$\mathrm{y} 2=1.0$

$\mathrm{x} 3=0.8000000$

y3 $=2.232239$

bn $=$ cubic_spline $(p, x 1, x 2, x 3, y 1, y 2, y 3)$

return

elseif (p.1t.1.74) then

$b n=-0.41579+1.8333 * p+2.6484 * p * * 2-1.0031 * p * * 3$

return

elseif (p.le.3.0)then

$\mathrm{x} 1=1.740000$

$\mathrm{y} 1=5.508093$

$\mathrm{x} 2=2.4$

y2 $=7.202996$

$\mathrm{x} 3=3.0$

$\mathrm{y} 3=7.903550$

bn $=$ cubic_spline $(p, x 1, x 2, x 3, y 1, y 2, y 3)$

return

else

$b n=8.22+1.10 * a \log (p / 4$.

endif

return

end 
function cubic_spline $(p, x 1, x 2, x 3, y 1, y 2, y 3)$

C Approximates function with the 3-nodes cubic spline

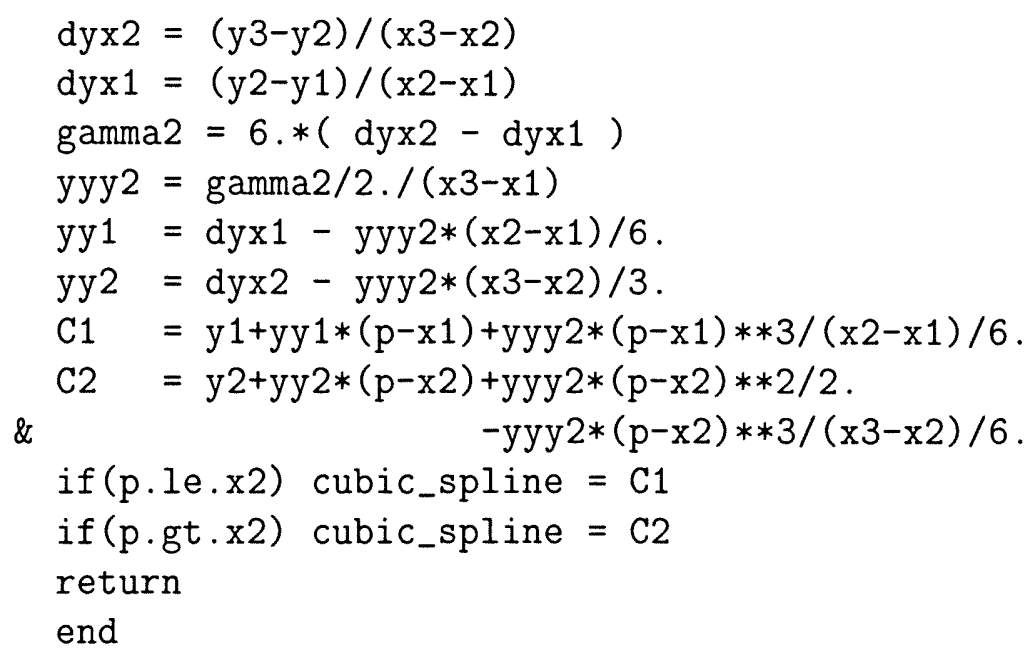

